\title{
Simulating the Nonadiabatic Relaxation Dynamics of 4-(N,N-Dimethylamino)benzonitrile (DMABN) in Polar Solution
}

Michal Kochman and Bo Durbeej

The self-archived postprint version of this journal article is available at Linköping University Institutional Repository (DiVA):

http://urn.kb.se/resolve?urn=urn:nbn:se:liu:diva-164526

N.B.: When citing this work, cite the original publication.

Kochman, M., Durbeej, Bo, (2020), Simulating the Nonadiabatic Relaxation Dynamics of 4-(N,NDimethylamino)benzonitrile (DMABN) in Polar Solution, Journal of Physical Chemistry A, 124(11), 2193-2206. https://doi.org/10.1021/acs.jpca.9b10588

Original publication available at:

https://doi.org/10.1021/acs.jpca.9b10588

Copyright: American Chemical Society

http://pubs.acs.org/ 


\title{
Simulating the Nonadiabatic
}

\section{Relaxation Dynamics of}

\section{4-(N,N-Dimethylamino)benzonitrile (DMABN) in Polar Solution}

\author{
Michał Andrzej Kochman*,,$+\ddagger$ and Bo Durbeej ${ }^{*, \dagger}$ \\ $\dagger$ Division of Theoretical Chemistry, Department of Physics, Chemistry and Biology (IFM), \\ Linköping University, 58183 Linköping, Sweden. \\ $\ddagger$ Department of Chemistry, University College London (UCL), 20 Gordon Street, London \\ WC1H 0A, United Kingdom \\ E-mail: m.kochman@ucl.ac.uk; bodur@ifm.liu.se
}

\begin{abstract}
The compound 4-(N,N-dimethylamino)benzonitrile (DMABN) represents the archetypal system for dual fluorescence, a rare photophysical phenomenon in which a given fluorophore shows two distinct emission bands. Despite extensive studies, the underlying mechanism remains the subject of debate. In the present contribution, we address this issue by simulating the excited-state relaxation process of DMABN as it occurs in polar solution. The potential energy surfaces for the system are constructed with the use of the additive QM/MM method, and the coupled dynamics of the electronic wavefunction and the nuclei is propagated with the semiclassical fewest switches surface hopping method. The DMABN molecule, which comprises the QM subsystem, is treated with the use of the second-order algebraic diagrammatic
\end{abstract}


construction $(\mathrm{ADC}(2))$ method with the imposition of spin-opposite scaling (SOS). It is verified that this level of theory achieves a realistic description of the excited-state potential energy surfaces of DMABN. The simulation results qualitatively reproduce the main features of the experimentally-observed fluorescence spectrum, thus allowing the unambiguous assignment of the two fluorescence bands: the normal band is due to the near-planar locally excited (LE) structure of DMABN, while the so-called 'anomalous' second band arises from the twisted intramolecular charge transfer (TICT) structure. The transformation of the LE structure into the TICT structure takes place directly via intramolecular rotation, and is not mediated by another excited-state structure. In particular, the oft-discussed rehybridized intramolecular charge transfer (RICT) structure, which is characterized by a bent nitrile group, does not play a role in the relaxation process.

\section{Background}

Understanding the photophysics of donor-acceptor compounds based on 4-aminobenzonitrile (ABN, see Figure 1 (a) for molecular structure) is one of the oldest challenges in the spectroscopy of organic molecules. In 1959, Lippert and coworkers ${ }^{1}$ first discovered that the ABN derivative 4-(N,N-dimethylamino)benzonitrile (DMABN, Figure 1 (b)) exhibits an intricate dual fluorescence process. At a short time delay (of the order of $0.5 \mathrm{ps}$ ) after the irradiation of its first photoabsorption band, DMABN shows a single fluorescence band whose position is fairly insensitive to solvent polarity; this is the so-called 'normal' band. In solvents of sufficiently high polarity, the intensity of the normal band decreases on a time scale of picoseconds, while simultaneously a second fluorescence band appears, which is red-shifted with respect to the normal band, and whose maximum is much more affected by solvent polarity. This latter band is known as the 'anomalous' band. Eventually, the emission profile stabilizes with both bands present; the intensity ratio of the anomalous and normal bands increases with increasing solvent polarity. The parent compound ABN shows 
Figure 1: Molecular structures of some aminobenzonitriles: (a) 4-aminobenzonitrile (ABN), (b) 4-( $N, N$-dimethylamino)benzonitrile (DMABN), (c) 5-cyano- $N$-methylindoline (CMI), (d) 6-cyanobenzquinuclidine (CBQ), and 1-tert-butyl-6-cyano-1,2,3,4-tetrahydroquinoline (NTC6).

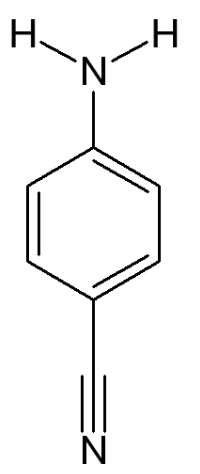

(a) $\mathrm{ABN}$

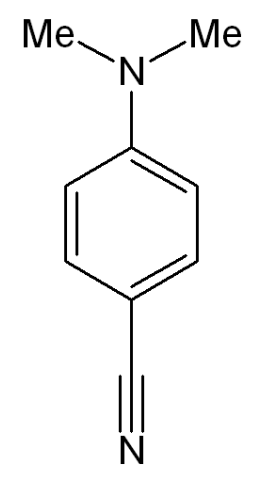

(b) DMABN

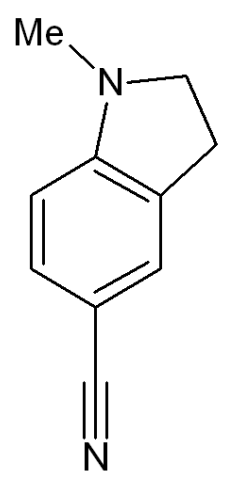

(c) $\mathrm{CMI}$

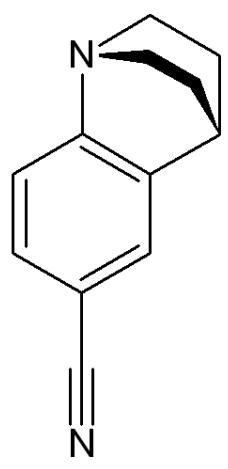

(d) CBQ

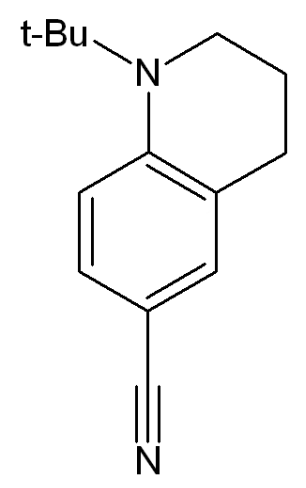

(e) NTC6

only the normal fluorescence - one of the prerequisites for the appearance of the anomalous band is that the electron-donating ability of the amino group must be enhanced by two alkyl substituents.

A few years later, Lippert et al. ${ }^{2}$ presented the earliest theoretical model for the dual fluorescence process, which has since become known as the state reversal model. As illustrated in Figure 2, the state reversal model postulates that DMABN has two closely spaced ${ }^{1} \pi \pi^{*}$-type states: the ${ }^{1} \mathrm{~L}_{\mathrm{b}}$ and ${ }^{1} \mathrm{~L}_{\mathrm{a}}$ states, in the terminology of Platt. ${ }^{3}$ The ${ }^{1} \mathrm{~L}_{\mathrm{a}}$ state is assumed to have a larger electric dipole moment than the ${ }^{1} \mathrm{~L}_{\mathrm{b}}$ state. In modern terminology, the ${ }^{1} \mathrm{~L}_{\mathrm{a}}$ state is an intramolecular charge transfer (ICT) state, whereas the ${ }^{1} \mathrm{~L}_{\mathrm{b}}$ state is a locally excited (LE) state. (For reference, the structures of the excited electronic states of DMABN are further characterized in Section S2 of the Supporting Information.) In a non-polar solvent, the ${ }^{1} \mathrm{~L}_{\mathrm{b}}$ state is lower in energy than the ${ }^{1} \mathrm{~L}_{\mathrm{a}}$ state. Under these conditions, emission occurs predominantly from the ${ }^{1} \mathrm{~L}_{\mathrm{b}}$ state (in accordance with Kasha's rule $^{4}$ ), giving rise to the normal fluorescence band. In a polar solvent, however, the solvent molecules may reorient in such a way as to reverse the energy ordering of the two excited 
Figure 2: A schematic illustration of the state reversal model of Lippert et al. ${ }^{2}$ GS is the singlet ground state of DMABN. The irradiation of the first photoabsorption band populates both the ${ }^{1} \mathrm{~L}_{\mathrm{b}}$ and ${ }^{1} \mathrm{~L}_{\mathrm{a}}$ excited states. Molecules excited initially into the ${ }^{1} \mathrm{~L}_{\mathrm{a}}$ state undergo rapid internal conversion (IC) into the ${ }^{1} \mathrm{~L}_{\mathrm{b}}$ state. In a non-polar solvent, fluorescence emission occurs only from the ${ }^{1} \mathrm{~L}_{\mathrm{b}}$ state (left panel). In a polar solvent, the solvent molecules around the DMABN molecule may reorient in such a way as to preferentially stabilize the ${ }^{1} \mathrm{~L}_{\mathrm{a}}$ state (right panel). This leads to the appearance of the anomalous fluorescence band. Adapted from Ref. ${ }^{2}$

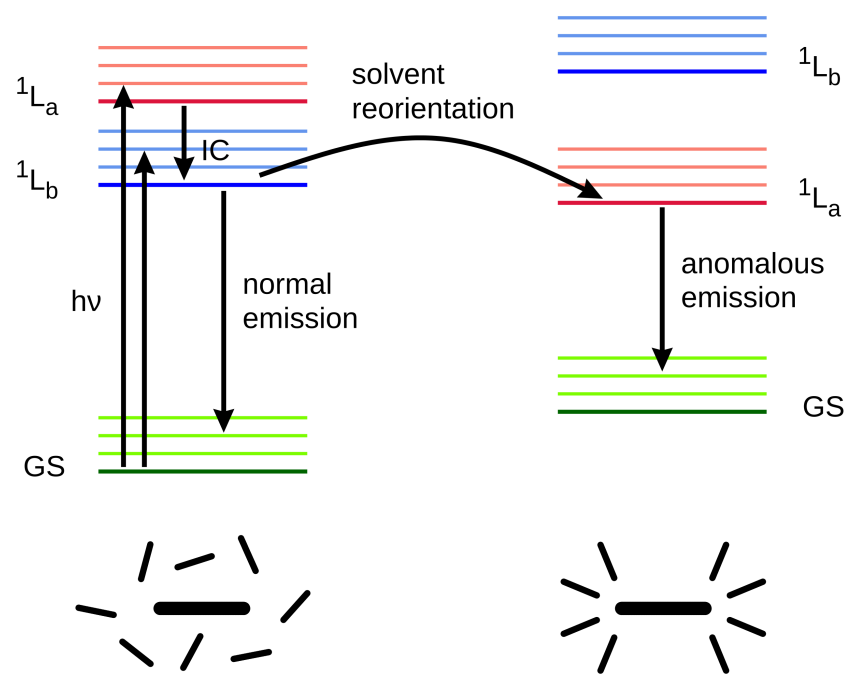

states. This enables emission from the ${ }^{1} \mathrm{~L}_{\mathrm{a}}$ state, which then leads to the appearance of the anomalous band. Thus, in the state reversal model, the key reaction coordinate for dual fluorescence is solvent reorientation.

The pioneering studies by Lippert and coworkers generated a great deal of interest in aminobenzonitriles and related donor-acceptor systems; for a detailed historical perspective on research in this area, we refer the reader to Refs. ${ }^{5-8}$ Over the years, the mechanism of dual fluorescence, and especially the nature of the species responsible for the anomalous fluorescence, has become the subject of substantial controversy, and a number of mutually exclusive hypotheses have been put forward to replace the state reversal model. In order to set the stage for subsequent discussion, we will briefly review the mechanisms that have most often been discussed in the literature, and some of the main arguments for and against them. For ease of reference, Figure 3 shows the ground-state structure of DMABN, and 
the various excited-state structures that have been proposed to be involved in the dual fluorescence process.

In 1973, the state reversal model was questioned by Rotkiewicz and coworkers. ${ }^{9}$ These authors recorded the fluorescence spectra of derivatives of DMABN in glycerol, a viscous solvent. Unexpectedly, it was found that the normal and anomalous fluorescence bands show a parallel polarization, in contradiction to the state reversal model, which requires the two bands to be mutually perpendicularly polarized. Moreover, it was noted that glycerol cannot undergo a substantial reorientation during the excited-state lifetime of DMABN. This was taken to indicate that the large Stokes shift of the anomalous band cannot be solely due to solvent reorientation.

In order to explain these observations, Rotkiewicz et al. ${ }^{9}$ instead proposed that the dual fluorescence arises from the presence of two excited-state structures which differ from one another in terms of molecular geometry. The normal fluorescence was ascribed to a non-polar structure in which the dimethylamino group lies roughly in the plane of the aromatic ring. This structure was later called the LE structure (see Figure 3 (b)). The anomalous emission, in turn, was tentatively attributed to a highly polar structure in which the dimethylamino group is oriented perpendicularly to the plane of the aromatic ring, and donates charge into the in-plane $\pi^{*}$-type orbital of the nitrile group. The term twisted ICT (TICT) was coined to describe this structure. Later on, theoretical studies have shown that the TICT structure does, in fact, exist, but its electronic structure is different than originally envisioned by Rotkiewicz et al. ${ }^{9}$ Specifically, these studies predicted the TICT structure to be a minimum on the potential energy surface (PES) of the ${ }^{1} \mathrm{~L}_{\mathrm{a}}$ state, with charge transfer occurring from the amino group into an out-of-plane $\pi^{*}$-type orbital that is delocalized over the aromatic ring as well as the nitrile group. ${ }^{10-12}$ Its geometry is illustrated in Figure 3 (c).

Additional evidence for the TICT model came from spectroscopic studies of ABN derivatives with varying degrees of constraint on the rotation of the amino group. ${ }^{13-16}$ 
Figure 3: A visual catalogue of the ground- and excited-state structures of DMABN. GS is the ground-state equilibrium geometry.

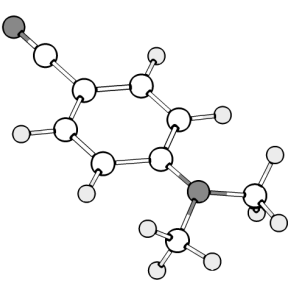

(a) GS

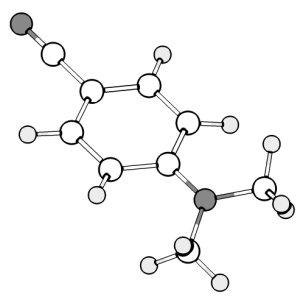

(b) LE

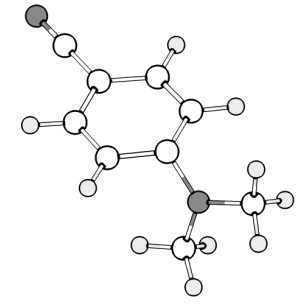

(c) TICT

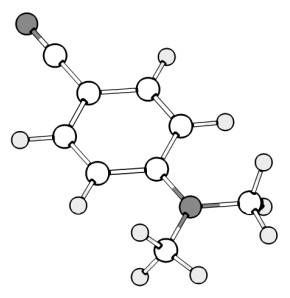

(d) PICT

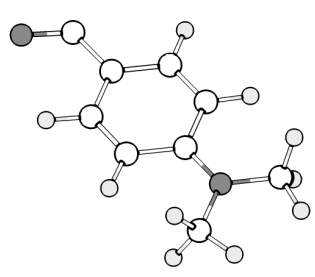

(e) RICT

For instance, 5-cyano- $N$-methylindoline (CMI, see Figure 1 (c)), in which the amino group is locked in a near-planar orientation, was found to exhibit only the normal fluorescence band across various solvents. ${ }^{16}$ In contrast, 6-cyanobenzquinuclidine (CBQ, Figure 1 (d)), whose amino group is fixed in a perpendicular orientation, exclusively shows the anomalous band. ${ }^{16}$

Despite its successes, the TICT model has not been universally accepted. In the early 1990s, Zachariasse and coworkers ${ }^{17,18}$ proposed a competing model for the dual fluorescence of aminobenzonitriles, according to which the anomalous fluorescence arises from a planar ICT (PICT, see Figure $3(\mathrm{~d})$ ) structure. In this model, the key structural coordinate that leads to the ICT structure is the planarization of the amino group. ${ }^{17,18} \mathrm{~A}$ small energy gap between the $\mathrm{S}_{1}\left({ }^{1} \mathrm{~L}_{\mathrm{b}}\right)$ and $\mathrm{S}_{2}\left({ }^{1} \mathrm{~L}_{\mathrm{a}}\right)$ states was cited as another important factor for dual fluorescence. ${ }^{17,18}$

In support of the PICT model, Zachariasse et al. ${ }^{19}$ pointed to the example of compounds such as 1-tert-butyl-6-cyano-1,2,3,4-tetrahydroquinoline (NTC6, see Figure 1 (e)) which 
undergo dual fluorescence despite being supposedly unable to undergo intramolecular rotation. However, later work by Hättig and coworkers ${ }^{20}$ cast doubt on that argument. These authors demonstrated, by means of ab initio calculations, that NTC6 is actually flexible enough to adopt TICT-type structures. The PICT model has also been criticized for its apparent inability to explain the occurrence of anomalous emission in pre-twisted aminobenzonitriles. $^{7}$

In 1996, Sobolewski and Domcke ${ }^{21,22}$ introduced a different model for the ICT structure of DMABN. Employing ab initio methods - geometry optimization at the level of configuration interaction singles ${ }^{23}$ (CIS) with single-point energies from complete active space second-order perturbation theory ${ }^{24,25}$ (CASPT2) - these authors found an excited-state structure with an in-plane orientation of the dimethylamino group, and a bent nitrile group (see Figure 3 (e)). In terms of electronic structure, this species is characterized by the excitation of an electron from an out-of-plane $\pi$-type orbital into the in-plane $\pi^{*}$-type orbital of the nitrile group (sometimes alternatively referred to as a $\sigma^{*}$-type orbital), and a large electric dipole moment. ${ }^{21,22}$ This electronic state is conventionally called the ${ }^{1} \pi \sigma^{*}$ state, and is distinct from the ${ }^{1} \mathrm{~L}_{\mathrm{a}}$ and ${ }^{1} \mathrm{~L}_{\mathrm{b}}$ states which featured in previous theoretical models. Dubbing this structure the rehybridized ICT (RICT) structure, Sobolewski and Domcke proposed that it is responsible for the anomalous fluorescence of DMABN. ${ }^{21,22}$

The RICT model has been criticized on the same grounds as the PICT model, namely, that it is unable to explain the experimentally-observed relationships between the structure and the optical properties of aminobenzonitriles. ${ }^{7}$ In later studies, Sobolewski, Sudholt, and Domcke ${ }^{26,27}$ discarded the RICT model in favour of the TICT model, concluding that the intramolecular rotation seems to be the relevant reaction coordinate for ICT.

Another point of controversy is the sequence of events in the mechanism of dual fluorescence. Within the last two decades, three kinetic models have been proposed to describe the relaxation process of DMABN following photoexcitation.

Kinetic model I, summarized in equation 1, was put forward by Zachariasse and 
coworkers ${ }^{28,29}$ on the basis of time-resolved fluorescence (TRF) and transient absorption (TA) measurements. This model is essentially phenomenological in approach, and does not make any statement regarding the geometry of the ICT structure. According to the model, after the initial photoexcitation, DMABN relaxes from the Franck-Condon (FC) geometry to the LE structure. ${ }^{28,29}$ Subsequently, the LE structure converts reversibly into the ICT structure. The LE and the ICT structures are responsible for the normal and the anomalous bands, respectively. ${ }^{28,29}$ In equation 1, as well as in the subsequent equations, the emissive structures are marked with squiggly arrows. $k_{a}$ and $k_{d}$ are the rate constants for the forward and reverse reactions, respectively.

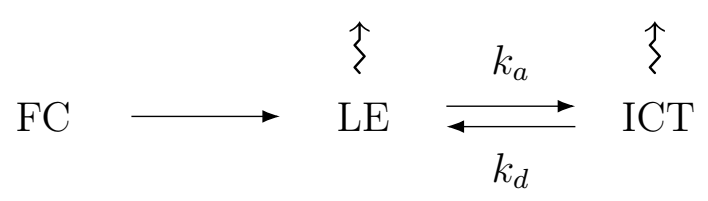

Kinetic model II, shown schematically in equation 2, was proposed by Fujiwara and coworkers, ${ }^{30}$ and incorporates the RICT structure found previously by Sobolewski and Domcke $^{21,22}$ as an intermediate photoproduct. Within this model, following the initial photoexcitation, the excited-state population branches off into two reaction channels, of which one leads to the LE structure, and the other to the RICT structure. ${ }^{30}$ This latter structure is characterized as non-fluorescent (spectroscopically dark), and acts as the precursor of the TICT structure, which is identified as the fluorescent ICT species. ${ }^{30}$

According to Fujiwara et al., ${ }^{30}$ the RICT structure is responsible for an intense excited-state absorption (ESA) feature near $700 \mathrm{~nm}$ in the TA spectrum of DMABN. This is justified by reference to earlier studies by Zgierski and Lim, ${ }^{31,32}$ who calculated the ESA spectrum of DMABN at the time-dependent density functional theory (TDDFT) level. However, the assignment of the ESA feature near $700 \mathrm{~nm}$ to the RICT structure has later been disputed. For example, Zachariasse et al. ${ }^{29}$ have demonstrated that similar ESA bands in the same spectral region also appear in the TA spectra of DMABN analogues in which the nitrile group is replaced with other electron-withdrawing substituents, and which, 
consequently, cannot adopt RICT-type structures. Moreover, Galván et al. ${ }^{33,34}$ simulated the ESA spectrum of DMABN at the CASPT2 level of theory, and concluded that the TA band in question may actually originate from the LE structure.

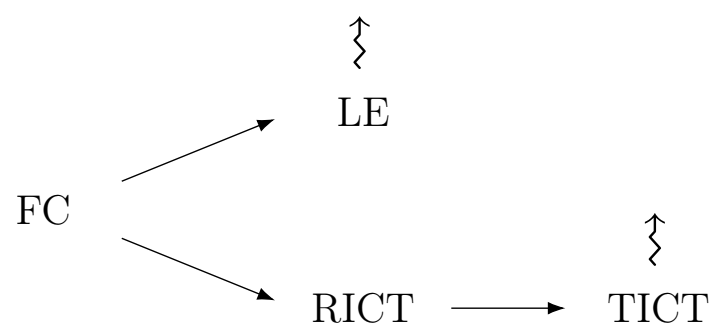

Kinetic model III, finally, shown in equation 3, was formulated by Coto and coworkers. ${ }^{35}$ It features as many as three ICT structures. One is the RICT structure, whose involvement is invoked to explain the intense ESA feature near $700 \mathrm{~nm} .{ }^{35}$ In this regard, kinetic model III is similar to kinetic model II of Fujiwara et al. ${ }^{30}$ However, Coto et al. ${ }^{35}$ argue that a fully twisted TICT structure, with a perpendicular orientation of the dimethylamino group, cannot be responsible for the anomalous band, as the oscillator strength for emission is negligibly low. Instead, these authors propose that emission preferentially occurs at a partially twisted ICT (pTICT) structure. ${ }^{35}$ The fully twisted TICT structure is also retained in kinetic model III, and some of the ESA features which appear in the TA spectrum are assigned to that structure. ${ }^{35}$

The model of Coto et al. ${ }^{35}$ envisions that after the initial photoexcitation, the excited-state population splits up to occupy the LE and the RICT structures. Afterwards, the LE structure converts into the pTICT structure, whereas the RICT structure transforms into the TICT structure.

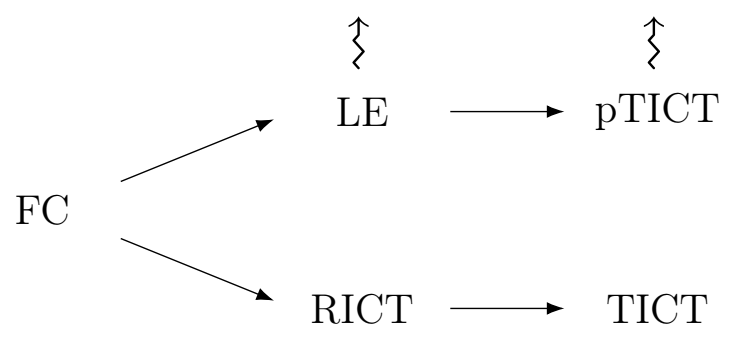


Although the kinetic aspects of the relaxation processes of aminobenzonitriles have been the subject of several computational studies, ${ }^{36-45}$ there is still insufficient data to conclusively support any one of the three kinetic models reviewed above. Many of the unanswered questions concern the role (if any) of the RICT structure. Experimentally, this structure is quite elusive due to its short lifetime and lack of detectable fluorescence emission. On the other hand, the question of its involvement in the relaxation process lends itself well to computational modeling. If the RICT structure plays a role in the mechanism, this fact should be reproduced by a simulation of the relaxation process.

A few years ago, Kochman and coworkers ${ }^{43}$ investigated the initial stage of the excited-state relaxation process of DMABN in the gas phase with the use of nonadiabatic molecular dynamics (MD) simulations. Presently, we extend the simulation setup used in that study to include an explicit solvent model, which enables us to construct a comprehensive theoretical picture of the relaxation dynamics of DMABN in polar solution, from the initial photoexcitation until the onset of dual fluorescence. The simulation results provide a direct test of the existing kinetic models of the dual fluorescence mechanism of DMABN.

The rest of the paper is organized as follows. First, we describe the simulation setup, and introduce the structural parameters with which we will monitor the relaxation dynamics. Subsequently, we examine the ground- and excited-state equilibrium geometries of DMABN. We then move on to discuss the simulated dynamics of this compound in the gas phase and in a water nanodroplet, as well as the resulting TRF spectra. Finally, we tie in the simulation results with experimental data on the relaxation process, and theoretical models of the mechanism of dual fluorescence. 


\section{Computational Methods}

\subsection{Overview}

For the sake of brevity, in the main body of the present paper we will include only a brief outline of the computational methodology. The detailed description of the simulation setup, and the rationale behind the choice of electronic structure method, are relegated to the Supporting Information.

The aim of our simulations is to model the excited-state relaxation process resulting from the irradiation of the lowest photoabsorption band of DMABN in polar solution. We do not attempt to reproduce any specific set of experimental conditions, but rather, we construct a somewhat idealized model of polar solvation. Specifically, we use water as the solvent, because its high polarity should ensure that the solvation effects will manifest themselves clearly. The PESs for the system are constructed with the use of the hybrid quantum mechanics/molecular mechanics (QM/MM) method, and its time-evolution is simulated by means of nonadiabatic MD. In order to quantify the solvation effects in the relaxation process, a reference set of nonadiabatic MD simulations is performed for the isolated DMABN molecule (i.e., for DMABN in the gas phase) with the same simulation parameters.

\subsection{Electronic Structure Methods}

The ground electronic state of DMABN was treated with the use of the Møller-Plesset perturbation method of second order (MP2), while its excited states were calculated with the use of the second-order algebraic diagrammatic construction $(\operatorname{ADC}(2))$ method. ${ }^{46,47}$ At all times, the spin-opposite scaling ${ }^{48,49}$ (SOS) procedure was imposed in both the MP2 and the $\mathrm{ADC}(2)$ calculations. As described in more detail in the Supporting Information, this measure improves the accuracy of $\mathrm{ADC}(2)$ for the relative energies of the LE and TICT structures of DMABN. In order to avoid confusion with the conventional implementations of the MP2 and $\mathrm{ADC}(2)$ methods (i.e., without a rescaling of the same- and opposite-spin 
contributions to the correlation energy), these calculations are referred to by the acronyms SOS-MP2 and SOS-ADC(2). The scaling factors were set to the values proposed in Ref. ${ }^{48}$ for use with the MP2 method: $c_{\mathrm{OS}}=1.3$ for the opposite-spin contributions, and $c_{\mathrm{SS}}=0$ for the same-spin contributions.

The SOS-MP2 and SOS-ADC(2) calculations were performed with the program Turbomole, version 6.3.1., ${ }^{50}$ taking advantage of the frozen core and resolution of the identity $^{51-54}$ approximations. A restricted Hartree-Fock (RHF) reference determinant was used. The cc-pVDZ basis set ${ }^{55}$ was employed with the default auxiliary basis set. ${ }^{56}$

\section{$2.3 \mathrm{QM} / \mathrm{MM}$ Calculations}

Due to the fact that periodic boundary conditions are not available in Turbomole, the solution phase was represented by placing the DMABN molecule at the center of a water nanodroplet containing 500 water molecules. The PESs for the system were generated with the use of the additive variant of the QM/MM method. ${ }^{57,58}$ In this scheme, the system $($ denoted $\mathbb{S}$ ) is partitioned into two subsystems which are treated at different levels of approximation. The electronic structure of the inner subsystem $(\mathbb{I})$ is explicitly included in the calculation. The outer subsystem $(\mathbb{O})$, in turn, is described with the use of a molecular mechanics (MM) force field.

As illustrated schematically in Figure 4, in the present case the inner subsystem consisted of the DMABN molecule, and the water molecules collectively comprised the outer subsystem. The electronic structure of DMABN was calculated at the SOS-ADC(2) level of electronic structure theory. The water molecules were treated with the flexible TIP3P water model as parameterized by Schmitt and Voth. ${ }^{59}$ In order to allow the electronic wavefunction of the DMABN molecule to adapt to the charge distribution of the solvent, the point charges of the water molecules were included in the Hamiltonian of the inner subsystem. The working 
Figure 4: Schematic illustration of the partitioning of the system (denoted $\mathbb{S}$ ) into the inner $(\mathbb{I})$ and outer $(\mathbb{O})$ subsystems. The diagram shows a cross-section of the 500-molecule water droplet enclosing the DMABN molecule. (In the actual simulations, the DMABN molecule is fully enclosed by the solvent.)

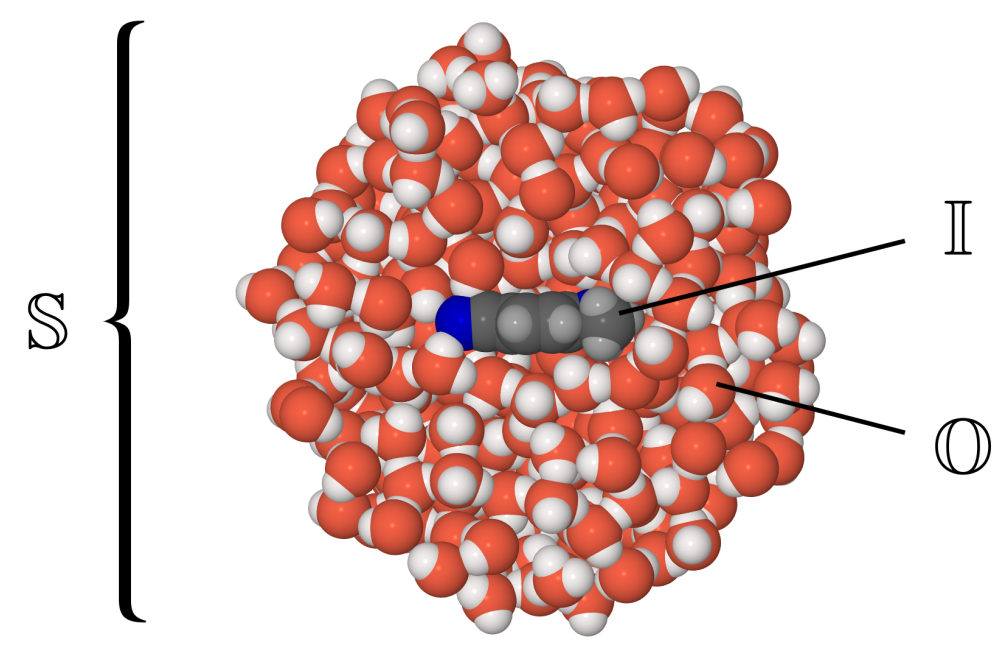

equation for the calculation of the potential energy of the system $\left(E_{\mathrm{QM} / \mathrm{MM}}(\mathbb{S})\right)$ was as follows:

$$
E_{\mathrm{QM} / \mathrm{MM}}(\mathbb{S})=E_{\mathrm{QM}}(\mathbb{I}, \mathbb{O})+E_{\mathrm{MM}}(\mathbb{O})+E_{\mathrm{QM}-\mathrm{MM}}(\mathbb{I}, \mathbb{O})
$$

Here, $E_{\mathrm{QM}}(\mathbb{I}, \mathbb{O})$ is the energy of the inner subsystem calculated at the QM level (specifically, SOS-ADC $(2))$ in the presence of the charge distribution of the outer subsystem. $E_{\mathrm{MM}}(\mathbb{O})$ is the energy of the outer subsystem evaluated at the MM level. Lastly, the QM-MM coupling term $E_{\mathrm{QM}-\mathrm{MM}}(\mathbb{I}, \mathbb{O})$ accounts for the van der Waals interactions between the inner and outer subsystems. (NB the electrostatic interactions between the two subsystems are accounted for in the term $E_{\mathrm{QM}}(\mathbb{I}, \mathbb{O})$, by virtue of the fact that the point charges of the water molecules are included in the QM Hamiltonian.) More details on the implementation can be found in the Supporting Information. 


\subsection{Nonadiabatic Molecular Dynamics}

In the nonadiabatic MD simulations, the simultaneous evolution of the electronic and the nuclear degrees of freedom was modeled via the fewest switches surface hopping (FSSH) method, ${ }^{60-63}$ adapted for use in combination with the hybrid QM/MM technique. Within the framework of the simulation model, the nuclear wavepacket of the system is represented by an ensemble of mutually independent semiclassical trajectories.

In each simulated trajectory, the dynamics of the nuclei is described by means of classical mechanics. In addition, the simulation explicitly includes the electronic structure of the solute and its evolution in time. To that end, the electronic wavefunction of the solute, $\Phi(\mathbf{r}, t ; \mathbf{R})$, is expressed as a linear combination of adiabatic electronic states $\left\{\phi_{j}(\mathbf{r} ; \mathbf{R})\right\}$ with time-dependent complex coefficients $\left\{a_{j}(t)\right\}$ :

$$
\Phi(\mathbf{r}, t ; \mathbf{R})=\sum_{j} a_{j}(t) \phi_{j}(\mathbf{r} ; \mathbf{R})
$$

Here, $\mathbf{r}$ denotes the electronic coordinates, and $\mathbf{R}=\mathbf{R}(t)$ is the trajectory followed by the nuclei. In the present case, the linear expansion 5 includes the $\mathrm{S}_{1}$ and $\mathrm{S}_{2}$ states. The requirement that $\Phi(\mathbf{r}, t ; \mathbf{R})$ satisfies the time-dependent electronic Schrödinger equation leads to the following system of coupled differential equations for the time-evolution of the expansion coefficients:

$$
\mathrm{i} \hbar \dot{a}_{k}=\sum_{j} a_{j}\left(\delta_{k j} E_{k}(\mathbf{R})-\mathrm{i} \hbar C_{k j}\right)
$$

where $\delta_{k j}$ denotes the Kronecker delta, $E_{k}(\mathbf{R})$ is the potential energy of the $k$-th adiabatic state, and $C_{k j}=\left\langle\phi_{k}\left|\frac{\partial}{\partial t}\right| \phi_{j}\right\rangle$ is the nonadiabatic coupling matrix element between states $k$ and $j$.

In each simulated trajectory, at any time the system is considered to occupy some current adiabatic state $n$ from among the states included in equation 5. The current state is selected according to its state population $\left|a_{n}\right|^{2}$. The nuclear dynamics is propagated according to 
Newton's equations of motion on the potential energy surface of that state. Nonadiabatic effects are accounted for by allowing the system to undergo a switch (or "hop") between the current state and another adiabatic state, which then becomes the new current state.

The FSSH simulations were carried out in a "wrapper" program, which was interfaced to Turbomole. At each timestep of the simulation, the wrapper program generated Turbomole input files, ran the dscf and ricc2 subprograms of Turbomole, then parsed the output, and propagated the nuclear and electronic equations of motion. The nonadiabatic coupling matrix elements which appear in equation 6 were calculated with with the use of the wavefunction overlap program of Plasser and coworkers. ${ }^{64,65} 50$ trajectories each were propagated for DMABN in the gas phase and in the water nanodroplet. For more details on the implementation, the reader is referred to Section S1 of the Supporting Information.

The sequence of events during the simulated dynamics was followed by monitoring several variables whose definitions we will now provide. As per the usual convention, the classical population of the $j$-th adiabatic state from among the states included in equation 5 is defined as the fraction of trajectories evolving in that state:

$$
P_{j}(t)=\frac{N_{j}(t)}{N_{\text {trajs }}}
$$

As illustrated in Figure 5 (a), the twisting (i.e., intramolecular rotation) of the dimethylamino group was tracked by following the value of a parameter $\tau$, defined as the average of the absolute values of the dihedral angles formed by atoms $\mathrm{C}^{\prime}-\mathrm{N} 1-\mathrm{C} 4-\mathrm{C} 3$, and $\mathrm{C}^{\prime \prime}-\mathrm{N} 1-\mathrm{C} 4-\mathrm{C} 5$ :

$$
\tau=\frac{1}{2}\left|\tau\left(\mathrm{C}^{\prime}-\mathrm{N} 1-\mathrm{C} 4-\mathrm{C} 3\right)\right|+\frac{1}{2}\left|\tau\left(\mathrm{C}^{\prime \prime}-\mathrm{N} 1-\mathrm{C} 4-\mathrm{C} 5\right)\right|
$$

Moreover, the bending of the nitrile group was followed by calculating the angle $\theta$ formed by atoms C1, C7, and N2 (see Figure $5(\mathrm{~b})$ ).

Lastly, the FSSH simulations were used as the basis for the calculation of the time-resolved fluorescence spectra of DMABN in the gas phase and in the water droplet. For either set of 
simulated trajectories, the emission intensity $\Gamma_{\mathrm{rad}}(E, t)$ at photon energy $E$ was calculated with the use of the semiclassical approximation of Crespo-Otero and Barbatti, ${ }^{66}$ adapted to the case of nuclear trajectories evolving in time:

$$
\Gamma_{\text {rad }}(E, t) \propto \sum_{i=1}^{N_{\text {trajs }}}\left[\Delta E_{n 0}\left(\mathbf{R}_{i}(t)\right)\right]^{2} f_{n 0}\left(\mathbf{R}_{i}(t)\right) g\left(E-\Delta E_{0 n}\left(\mathbf{R}_{i}(t)\right)\right)
$$

Here, $N_{\text {trajs }}$ is the number of simulated trajectories, $\mathbf{R}_{i}(t)$ is the geometry of the system at time $t$ along the $i$-th trajectory, $\Delta E_{n 0}\left(\mathbf{R}_{i}(t)\right)$ is the vertical energy difference between the currently occupied state $(n)$ and the ground state, $f_{n 0}\left(\mathbf{R}_{i}(t)\right)$ is the corresponding oscillator strength, and $g\left(E-\Delta E_{0 n}\left(\mathbf{R}_{i}(t)\right)\right)$ is a line shape function centered at $\Delta E_{n 0}\left(\mathbf{R}_{i}(t)\right)$. In order to obtain a smooth spectrum, and also to compensate for the relatively low number of simulated trajectories, we elected to apply a Gaussian line shape function with a high standard deviation value of $0.25 \mathrm{eV}$. Moreover, at the post-processing stage, the raw simulated spectrum obtained from equation 8 was subjected to a Gaussian blur in the time domain with a standard deviation of 25 fs.

Figure 5: Schematic illustration of (a) the intramolecular rotation parameter $\tau$ and (b) the nitrile group bending angle $\theta$.

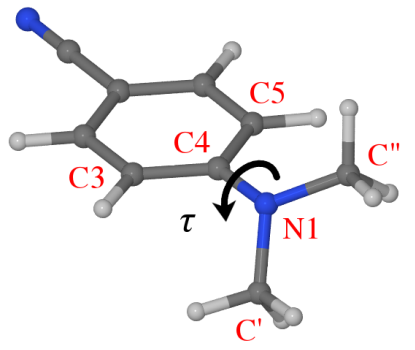

(a) dimethylamino group twisting angle, $\tau$

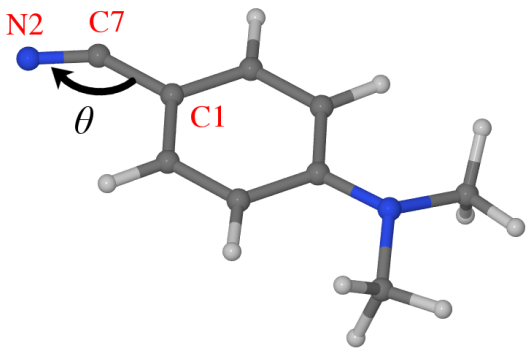

(b) nitrile group bending angle, $\theta$ 


\section{Results and Discussion}

\subsection{Equilibrium Geometries}

Our first order of business will be to examine the ground- and excited-state structures of the isolated DMABN molecule. The equilibrium geometries obtained at the SOS-MP2 and SOS-ADC(2) level of theory are illustrated in Figure 6, while Table 1 lists their relative energies and key properties. Following the convention introduced by Gómez and coworkers, ${ }^{12}$ we prefix the acronym of each molecular structure with the label of the adiabatic state on which it was optimized. For example, $\mathrm{S}_{1}$-LE denotes the minimum on the $\mathrm{S}_{1}$ state that corresponds to the locally excited structure.

We find that the ground-state equilibrium geometry of DMABN ( $\mathrm{S}_{0}-\mathrm{GS}$, Figure 6 (a)) is near-planar except for the slightly pyramidalized dimethylamino group nitrogen. This is in agreement with previous studies ${ }^{11,43}$ which reported geometry optimizations at the MP2 and second-order approximate coupled-cluster ${ }^{67}(\mathrm{CC} 2)$ levels of electronic structure theory. In the electronic ground state, DMABN has only a moderately large electric dipole moment of $6.62 \mathrm{D}$.

Two minima are found on the PES of the $\mathrm{S}_{1}$ state. The first corresponds to the LE structure $\left(\mathrm{S}_{1}\right.$-LE, Figure $\left.6(\mathrm{~b})\right)$, and the second is the TICT structure $\left(\mathrm{S}_{1}\right.$-TICT, 6 (c)). The $\mathrm{S}_{1}$-LE structure is the lower in energy of the two. At this structure, the $\mathrm{S}_{1}$ state has $\mathrm{L}_{\mathrm{b}}$ diabatic character. The dimethylamino group nitrogen is planarized, and the dimethylamino group as a whole adopts a slightly twisted orientation with respect to the six-membered ring. The overall nuclear geometry has ideal $C_{2}$ symmetry. The electric dipole moment of the $\mathrm{S}_{1}$-LE structure is $8.57 \mathrm{D}$, somewhat larger in magnitude than that of the $\mathrm{S}_{0^{-}}$-GS structure.

The $\mathrm{S}_{1}$-TICT structure lies adiabatically $0.119 \mathrm{eV}$ higher in energy than the $\mathrm{S}_{1}$-LE structure. (When zero-point vibrational energy corrections are included, the energy difference is $0.148 \mathrm{eV}$.) On the subject of the energy ordering of the $\mathrm{S}_{1}-\mathrm{LE}$ and $\mathrm{S}_{1}$-TICT structures, it should be noted that the relative energies of the two structures are sensitive to 
Figure 6: Ground- and excited-state equilibrium geometries of the isolated DMABN molecule as optimized at the SOS-MP2/cc-pVDZ and SOS-ADC(2)/cc-pVDZ levels of theory. Selected bond distances are marked in units of $\AA$.

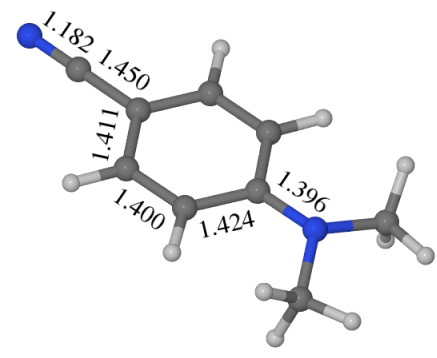

(a) $\mathrm{S}_{0}-\mathrm{GS}$

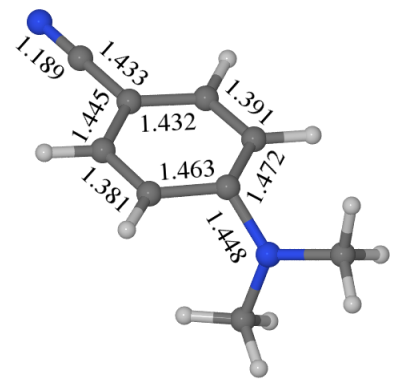

(c) $\mathrm{S}_{1}$-TICT

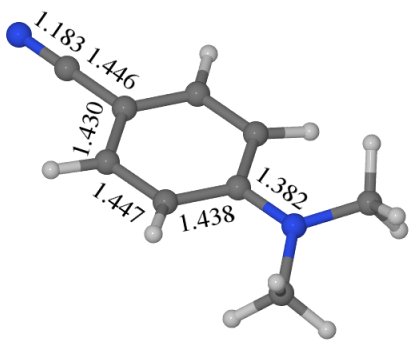

(b) $\mathrm{S}_{1}$-LE

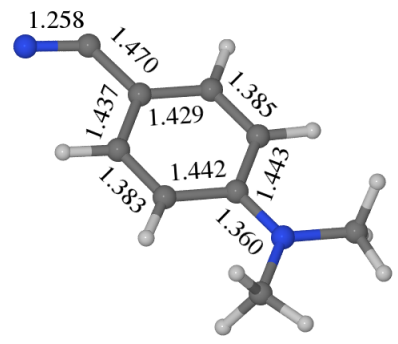

(d) $\mathrm{S}_{1}$-RICT

the level of electronic structure theory. In particular, the conventional variants of CC2 and $\mathrm{ADC}(2)$ methods (without spin-component scaling) erroneously place the $\mathrm{S}_{1}$-TICT structure lower in energy than the $\mathrm{S}_{1}$-LE structure. ${ }^{11,43}$ The application of the SOS procedure corrects this error. In this regard, $\operatorname{SOS}-\operatorname{ADC}(2)$ is clearly superior to conventional $\operatorname{ADC}(2)$.

Table 1: Adiabatic energies $(E)$, magnitudes of (orbital-relaxed) dipole moment $(\mu)$, and geometric parameters of the ground- and excited-state equilibrium geometries of DMABN, as optimized with the use of the SOS-MP2 and SOS-ADC(2) methods. The adiabatic energy values are quoted relative to the energy of the $\mathrm{S}_{0^{-}} \mathrm{GS}$ structure.

\begin{tabular}{lllll}
\hline Structure & $E, \mathrm{eV}$ & $\mu, \mathrm{D}$ & $\tau,{ }^{\circ}$ & $\theta,^{\circ}$ \\
\hline $\mathrm{S}_{0}-\mathrm{GS}$ & 0 & 6.62 & 17.0 & 180.0 \\
$\mathrm{~S}_{1}-\mathrm{LE}$ & 4.238 & 8.57 & 16.4 & 180.0 \\
$\mathrm{~S}_{1}-\mathrm{TICT}$ & 4.357 & 11.91 & 57.0 & 179.8 \\
$\mathrm{~S}_{1}-\mathrm{RICT}^{a}$ & 5.547 & 13.55 & 0.0 & 125.6 \\
\hline
\end{tabular}

${ }^{a}$ First-order saddle point on the PES of the $\mathrm{S}_{1}$ state. 
Figure 7: Schematic energy level diagram for the isolated DMABN molecule. The horizontal bars represent the energies of the relevant adiabatic electronic states at the ground- and excited-state equilibrium geometries. The diabatic character of each state is indicated with the use of color. The black bullseye symbol marks the electronic state in which a given structure was optimized. For the sake of clarity, from among the high-energy excited states of DMABN, only the ${ }^{1} \pi \sigma^{*}$ state is included in this diagram.

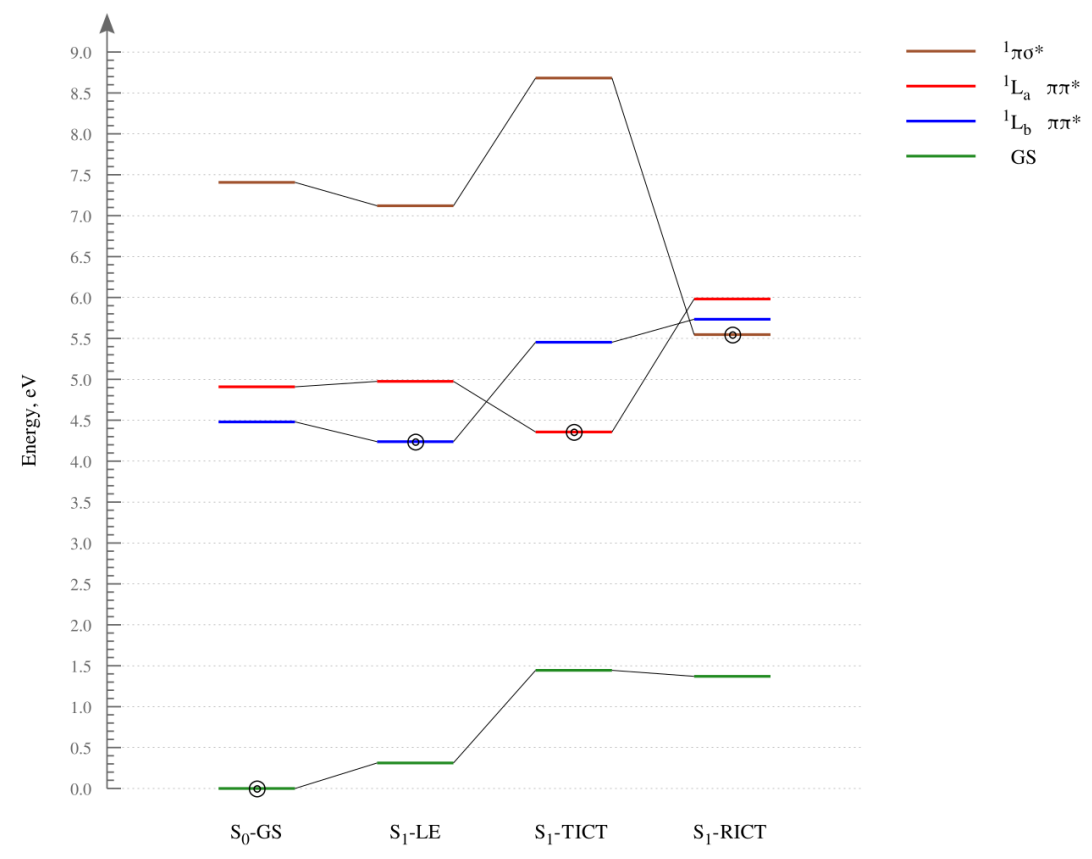

At the $\mathrm{S}_{1}$-TICT structure, the $\mathrm{S}_{1}$ state is characterized by $\mathrm{L}_{\mathrm{a}}$ diabatic character, and exhibits a fairly large electric dipole moment of $11.91 \mathrm{D}$. As pointed out in Ref., ${ }^{43}$ the $\mathrm{S}_{1}$-TICT structure features a shift of electron density from the dimethylamino group nitrogen onto carbon atom C4. Consequently, atom C4 has partial carbanionic character, and adopts a pyramidalized geometry. The C4-N1 bond is pointing away from the plane of the six-membered ring. The dimethylamino group adopts a skewed orientation in relation to the ring, such that the intramolecular rotation parameter $\tau$ takes a value of $57.0^{\circ}$. Due to the fact that the orientation of the methyl group is quite far from perpendicular, the equilibrium geometry obtained with the $\operatorname{SOS}-\mathrm{ADC}(2)$ method is best described as partially twisted (pTICT-like). This is in contrast to what is seen with the conventional CC2 and ADC(2) methods, both of which predict a near-perpendicular orientation of the dimethylamino 
group. ${ }^{11,43}$ In other respects, however, the equilibrium geometry found with SOS-ADC(2) closely resembles those obtained with the conventional $\mathrm{CC} 2$ and $\mathrm{ADC}(2)$ methods. Thus, the fact that $\mathrm{SOS}-\mathrm{ADC}(2)$ predicts a partially, rather than fully, twisted equilibrium geometry does not represent a serious failing on the part of that method. In any case, as we shall see when discussing the results of the nonadiabatic MD simulations, the TICT structure is quite flexible. In the water droplet, the simulated trajectories explore a range of TICT-type conformations, from partially to fully twisted (with $\tau \approx 90^{\circ}$ ). Thus, the exact orientation of the dimethylamino group at the $\mathrm{S}_{1}$-TICT equilibrium geometry is only of relatively minor importance.

We were unable to locate any further minima on the PES of the $\mathrm{S}_{1}$ adiabatic state beyond the $\mathrm{S}_{1}$-LE and $\mathrm{S}_{1}$-TICT structures. In particular, the PICT structure does not correspond to a minimum on the $\mathrm{S}_{1} \mathrm{PES}$. As regards the RICT structure, it, too, does not correspond to a minimum, but there does exist a first-order saddle point on the $\mathrm{S}_{1}$ PES where the $\mathrm{S}_{1}$ state has ${ }^{1} \pi \sigma^{*}$ diabatic character. This structure is illustrated in Figure $6(\mathrm{~d})$. In terms of overall geometry, it resembles the RICT structure as reported in previous theoretical studies, ${ }^{12,68,69}$ and for this reason, we regard it as the best approximation to the RICT structure achievable with $\operatorname{SOS}-\operatorname{ADC}(2)$.

The nuclear geometry at the $\mathrm{S}_{1}$-RICT structure has ideal $C_{s}$ symmetry. The normal mode with the imaginary frequency is antisymmetric with respect to reflection in the molecular plane of symmetry, and corresponds to an out-of-plane deformation of the heavy-atom skeleton. As can be seen from Figure 7, under gas-phase conditions, the $\mathrm{S}_{1}$-RICT structure lies as much as $0.638 \mathrm{eV}$ above the energy of the bright $\mathrm{S}_{2}\left({ }^{1} \mathrm{~L}_{\mathrm{a}}\right)$ state at the ground-state equilibrium geometry. This makes it essentially inaccessible under gas-phase conditions, assuming the molecule is excited initially into the $\mathrm{S}_{2}\left({ }^{1} \mathrm{~L}_{\mathrm{a}}\right)$ state.

While the $\mathrm{S}_{1}$-RICT structure is not expected to play a role in the relaxation dynamics of DMABN in the gas phase, the situation is less clear-cut in polar solution. The $\mathrm{S}_{1}$-RICT structure is characterized by a relatively large electric dipole moment of $13.55 \mathrm{D}$, and may 
benefit from stabilizing interactions with the polar solvent. It is conceivable that during the relaxation process in the polar solution phase, the molecule can temporarily assume a $\mathrm{S}_{1}$-RICT-type structure before relaxing further to another excited-state structure, and this possibility provides part of the motivation for the nonadiabatic MD simulations performed in the present study.

In closing this section, we note that due to the fact that at the SOS-ADC(2) level, the $\mathrm{S}_{1}$-RICT structure exists not as a minimum, but as a first-order saddle point on the PES of the $\mathrm{S}_{1}$ state, it is not expected to be able to trap the excited-state population for an extended period of time. For this reason, if the molecule was to at least briefly adopt a $\mathrm{S}_{1}$-RICT-type geometry during the simulated dynamics, we would consider that sufficient evidence for the involvement of the $\mathrm{S}_{1}$-RICT structure in the relaxation mechanism.

\subsection{Relaxation Dynamics in the Gas Phase}

We now move on to discuss the relaxation process of DMABN in the gas phase. Figure 8 (a) shows the classical populations of the $S_{1}$ and $S_{2}$ adiabatic states. The inset to the right is an enlarged view of the initial $100 \mathrm{fs}$ period after photoexcitation. Panel (b), in turn, presents a histogram of the intramolecular torsion angle $\tau$ as a function of time. Lastly, panel (c) is a histogram of the nitrile group bending angle $\theta$, again as a function of time.

At the time of the initial photoexcitation $(t=0)$, the ensemble of trajectories which represents the nuclear wavepacket was localized in a narrow region of configuration space around the ground-state equilibrium geometry. The twisting angle $\tau$ was distributed in the range of $0^{\circ}$ to $30^{\circ}$, while the nitrile group bending angle was distributed in the range of $160^{\circ}$ to $180^{\circ}$. (The spread in the values of $\tau$ and $\theta$ reflects the spatial distribution of the nuclear wavefunction of the molecule in its electronic ground state.) All 50 trajectories comprising the ensemble began in the $\mathrm{S}_{2}$ state.

The 30 fs-long period of time immediately following photoexcitation was marked by rapid population transfer from the $\mathrm{S}_{2}$ state into the $\mathrm{S}_{1}$ state. By $t=30 \mathrm{fs}$, the classical population 
of the $\mathrm{S}_{2}$ state had decayed to 6 trajectories (12\% of the total number). The occurrence of internal conversion from $\mathrm{S}_{2}$ to $\mathrm{S}_{1}$ during the first few tens of femtoseconds after the initial photoexcitation is in line with the results of previous FSSH simulations of the relaxation dynamics of DMABN in the gas phase, employing the $\mathrm{ADC}(2)^{43}$ and $\mathrm{TDDFT}^{41}$ methods, as well as ab initio multiple spawning (AIMS) simulations based on TDDFT. ${ }^{44}$

Following the initial 30 fs-long phase, and for the remainder of the simulation period of $1.2 \mathrm{ps}$, the ensemble of trajectories predominantly occupied the $\mathrm{S}_{1}$ state, though individual trajectories occasionally returned to the $\mathrm{S}_{2}$ state for up to a few tens of femtoseconds at a time. This tended to happen when the molecule approached the $\mathrm{S}_{2} / \mathrm{S}_{1}$ conical intersection seam while in the $\mathrm{S}_{1}$ state, which led to strong nonadiabatic coupling to the $\mathrm{S}_{2}$ state. Because of these brief excursions into the $\mathrm{S}_{2}$ state, its classical population did not decay to zero, but instead fluctuated continuously around an average value of 0.06 .

After the internal conversion from $\mathrm{S}_{2}$ to $\mathrm{S}_{1}$, most of the simulated trajectories became trapped in the potential energy well around the $\mathrm{S}_{1}$-LE minimum on the $\mathrm{S}_{1}$ state, and stayed there for the remainder of the simulation period. This is readily apparent from an inspection of Figure 8 (b), which shows the distribution of the twisting angle $\tau$. Until around $t=600 \mathrm{fs}$, the ensemble of trajectories was almost entirely contained in the $0-30^{\circ}$ bin of $\tau$, which is consistent with the $\mathrm{S}_{1}$-LE structure. After $t=600 \mathrm{fs}$, a few trajectories left the $0-30^{\circ}$ bin, and entered the $30-60^{\circ}$ bin; this may be in part due to a leakage of zero-point energy from the fast vibrational modes of the molecule into the intramolecular rotation mode. This effect is a simulation artifact resulting from the nuclei being treated as classical particles. ${ }^{70-72}$ In any case, the occurrence of intramolecular rotation is relatively rare, and sets in only late in the simulation period.

Only one of the simulated trajectories can be considered to have escaped the potential energy well around the $\mathrm{S}_{1}$-LE minimum, and crossed over to the well of the $\mathrm{S}_{1}$-TICT minimum. In Figure 8 (b), this event is marked with small black arrows. The single trajectory that escaped the $\mathrm{S}_{1}$ - LE minimum can be seen traversing the $60-90^{\circ}, 90-120^{\circ}$, 
and $120-150^{\circ}$ bins of $\tau$ as the dimethylamino group continues its rotation.

The low incidence of intramolecular rotation requires some comment. Several previous studies $^{41,43-45}$ have reported dynamical (time-resolved) simulations of the relaxation process of DMABN under gas-phase conditions. In those studies where the relaxation dynamics was propagated for at least a few hundred femtoseconds (Refs. ${ }^{41,43,44}$ ), a substantial fraction of the excited-state population did undergo intramolecular rotation. However, all of the works just cited employed electronic structure methods which are known to artificially stabilize the $\mathrm{S}_{1}$-TICT structure relative to the $\mathrm{S}_{1}$-LE structure. This problem was already recognized in Refs. ${ }^{43,44}$ In view of the above, the tendency towards intramolecular rotation observed in these studies must be viewed with caution. Most likely, this result was an artifact resulting from inaccuracies in the PESs on which the dynamics was propagated.

In summary, trapping in the $\mathrm{S}_{1}$-LE structure was by far the predominant outcome of the simulations. Only one of the 50 simulated trajectories adopted the twisted $\mathrm{S}_{1}$-TICT structure. No other excited-state structures were formed in the course of the gas-phase simulations. In particular, the involvement of the $\mathrm{S}_{1}$-RICT structure in the gas-phase relaxation process can be ruled out on the grounds that there were no instances of the nitrile group adopting a substantially bent geometry (see Figure 8 (c)).

Accompanying this narrative, as part of the Supporting Information we provide animations of four representative gas-phase trajectories. Each animation covers the initial 1 ps of the given trajectory. The panel on the bottom shows the energy gaps between the $\mathrm{S}_{1}$ and $\mathrm{S}_{2}$ states, and the $\mathrm{S}_{0}$ state. The passage of time is indicated with a vertical black line moving along the time axis. The currently occupied state is marked with a black circle. 
Figure 8: Time-evolution of electronic structure and molecular geometry during the excited-state relaxation dynamics of DMABN in the gas phase. (a) Classical populations of the $S_{1}$ and $S_{2}$ states. The inset on the right-hand side shows the earliest 100 fs-long period of the simulations. (b) Distribution of parameter $\tau$, which describes the torsion of the dimethylamino group. The arrows indicate the single trajectory which escapes the potential energy basin of the $\mathrm{S}_{1}$-LE structure. (c) Distribution of parameter $\theta$, which describes the bending of the nitrile group.

(a) Classical populations

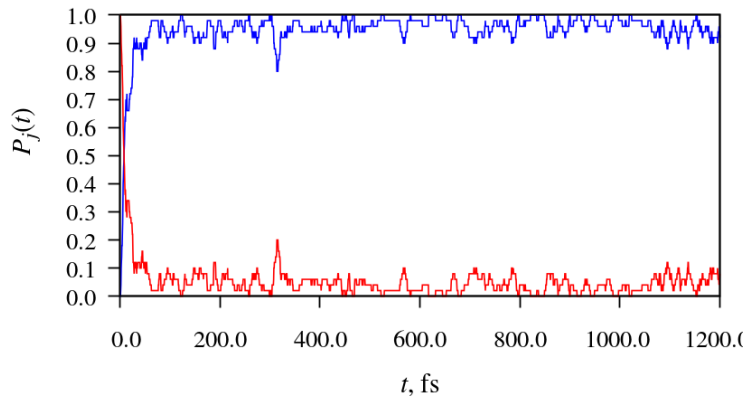

(b) Distribution of $\tau$

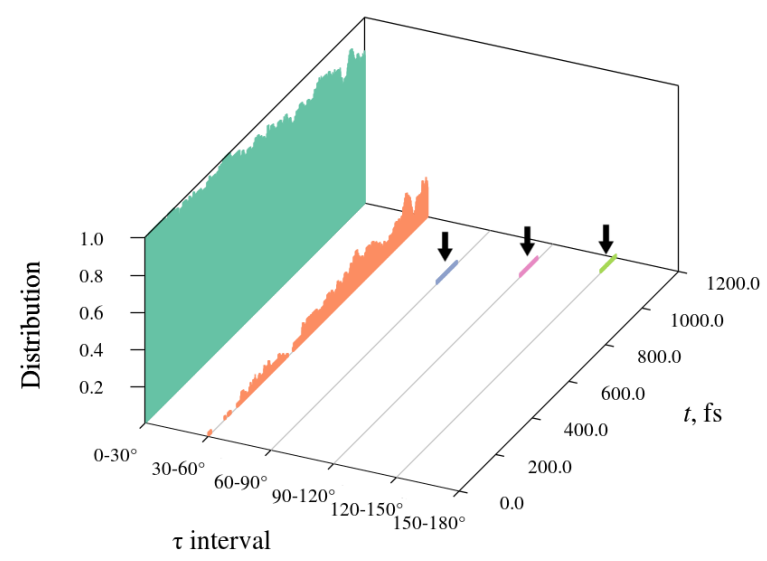

(initial $100 \mathrm{fs}$ )

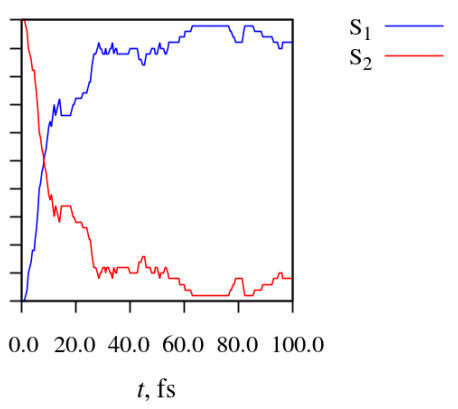

(c) Distribution of $\theta$

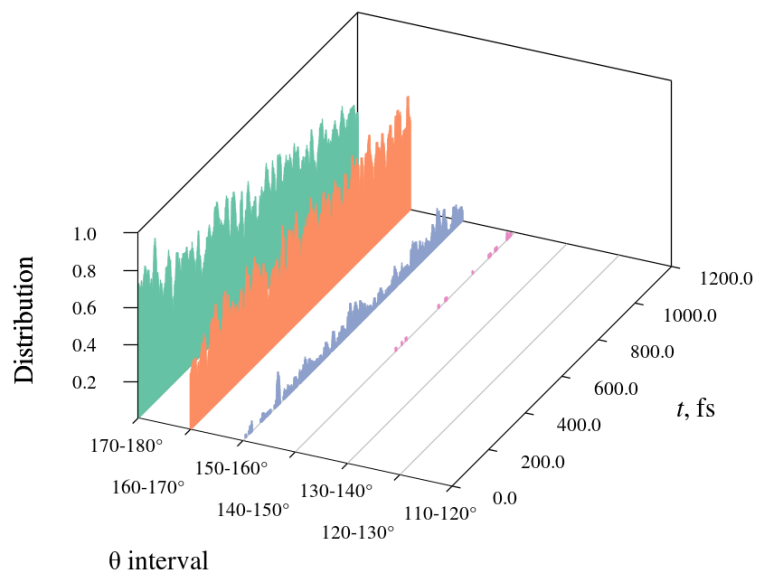

\subsection{Relaxation Dynamics in the Water Droplet}

Having examined the relaxation process of DMABN in the gas phase, we now move on to the more complex case of DMABN in the 500-molecule water droplet, which serves as a model of the polar solution phase. The results of these simulations are summarized in Figure 9, which is organized analogously to Figure 8.

At the outset of the simulated dynamics, the geometries of the DMABN molecule in the ensemble of simulated trajectories were clustered around the $\mathrm{S}_{0^{-}}$GS geometry. 44 of the 50 
simulated trajectories were initially occupying the $\mathrm{S}_{2}$ state, and the remaining 6 were in the $\mathrm{S}_{1}$ state.

As can be seen from Figure 9 (a), during the initial 20 fs-long period of time following photoexcitation, the ensemble of trajectories underwent internal conversion to the $\mathrm{S}_{1}$ state in much the same way as in the gas-phase simulations. By $t=20 \mathrm{fs}$, all 44 trajectories that began in the $\mathrm{S}_{2}$ state had hopped to the $\mathrm{S}_{1}$ state. From then on, the system mainly evolved in the $\mathrm{S}_{1}$ state. Due to intermittent hopping of trajectories from the $\mathrm{S}_{1}$ state into the $\mathrm{S}_{2}$ state, and then back to the lower state, the classical population of the $\mathrm{S}_{2}$ state fluctuated just above zero. As a side note, careful inspection of Figure 9 (a) reveals that these short-lived excursions into the $\mathrm{S}_{2}$ state gradually became rarer with the passage of time. We will return to this point later on in this section.

The internal conversion event took place while the DMABN molecule was still close to the near-planar Franck-Condon geometry. Shortly afterwards, however, and in sharp contrast to what is seen in the gas phase, the ensemble of simulated trajectories began to spread out along the intramolecular twisting coordinate (see Figure 9 (b)). By $t=300 \mathrm{fs}$, the first trajectories had entered the $60-90^{\circ}$ bin of $\tau$, which we interpret as the formation of the twisted $\mathrm{S}_{1}$-TICT structure. The population of the $60-90^{\circ}$ bin, and later also the $90-120^{\circ}$ bin, subsequently continued to grow. The build-up of excited-state population in these two bins is especially apparent from around $t=600$ fs. Although the simulation period of $1.2 \mathrm{ps}$ is too short for the ensemble of trajectories to approach thermodynamic equilibrium, it is clear that a significant proportion of the excited-state population settles in the $60-90^{\circ}$ and $90-120^{\circ}$ bins of $\tau$. At the time that the simulations were concluded, these two bins taken together held 33 of the 50 simulated trajectories ( $66 \%$ of the total number). Visual inspection suggests that the simulated trajectories generally did not remain in the $30-60^{\circ}$ bin for an extended period of time. It seems that the population of the $30-60^{\circ}$ bin consisted mainly of trajectories which were in transit from the $0-30^{\circ}$ bin into the $60-90^{\circ}$ and $90-120^{\circ}$ bins.

The formation of the $\mathrm{S}_{1}$-TICT structure was the reason for the gradual decline in the 
Figure 9: Time-evolution of electronic structure and molecular geometry during the excited-state relaxation dynamics of DMABN in the 500-molecule water droplet. (a) Classical populations of the $\mathrm{S}_{1}$ and $\mathrm{S}_{2}$ states. The inset on the right-hand side shows the earliest 100 fs-long period of the simulations. (b) Distribution of parameter $\tau$, which describes the torsion of the dimethylamino group. (c) Distribution of parameter $\theta$, which describes the bending of the nitrile group.

(a) Classical populations

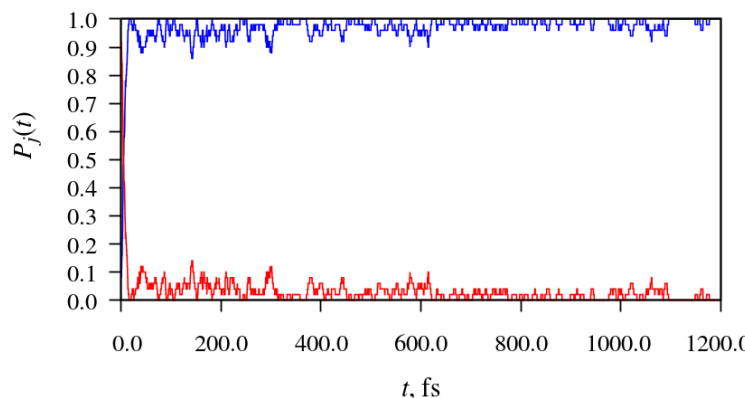

(b) Distribution of $\tau$

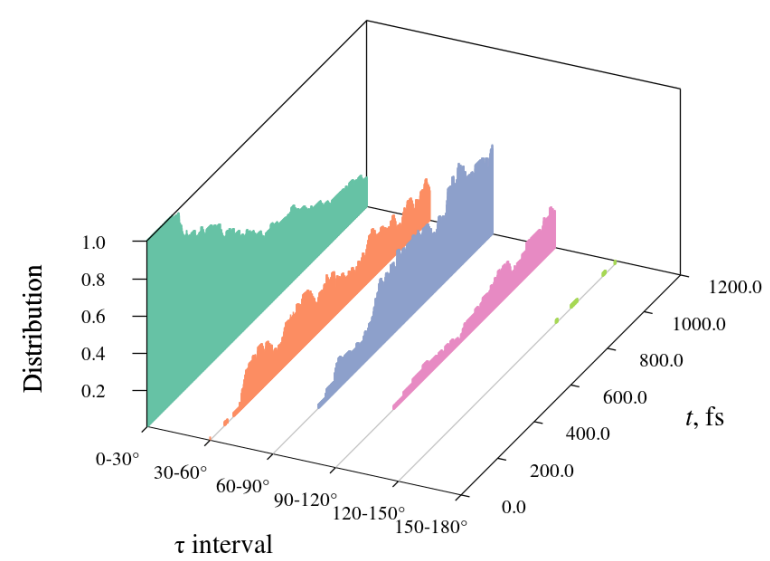

(initial $100 \mathrm{fs}$ )

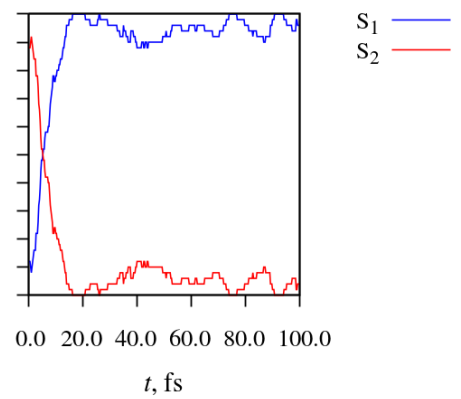

(c) Distribution of $\theta$

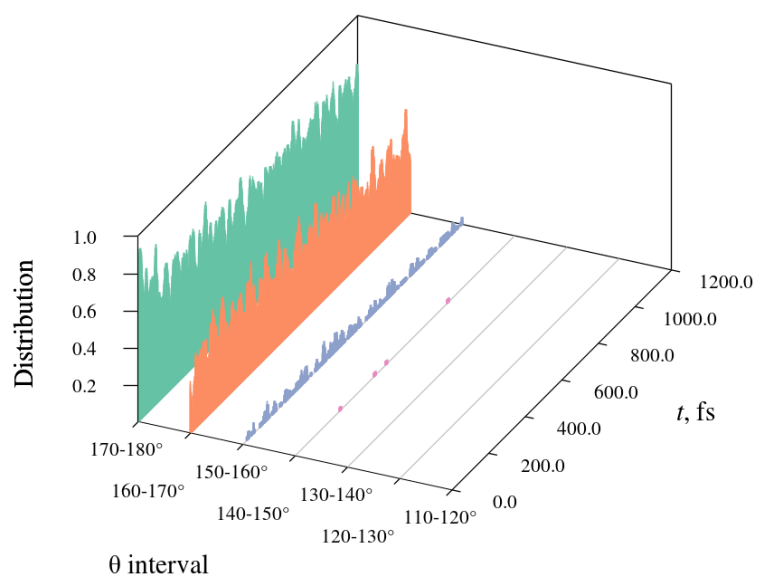

brief excursions of individual trajectories into the $\mathrm{S}_{2}$ state, which was mentioned earlier on in this section. At the $\mathrm{S}_{1}$-TICT structure, there is a large energy gap between the $\mathrm{S}_{2}$ and $\mathrm{S}_{1}$ states. As a consequence, nonadiabatic coupling between the $\mathrm{S}_{2}$ and $\mathrm{S}_{1}$ states is vanishingly weak, making an upward hop from the $\mathrm{S}_{1}$ state into the $\mathrm{S}_{2}$ state very unlikely.

Once in the $\mathrm{S}_{1}$-TICT structure, the DMABN molecule does not undergo any further transformations within the time scale of the simulation. This finding suggests that the $\mathrm{S}_{1}$-TICT structure is the final product of the simulated relaxation process. We did not observe any instances in which the DMABN molecule adopted a geometry similar to the 
$\mathrm{S}_{1}$-RICT structure, with a bent nitrile group. Indeed, as can be deduced from Figure 9 (a), throughout the simulated dynamics the nitrile group underwent only small-amplitude bending vibrations.

Animations of four representative simulated trajectories of DMABN in the water droplet are included in the Supporting Information. Each animation shows the solute molecule and the nearest water molecules around it. (More specifically, the animation shows all water molecules whose oxygen atoms approach the DMABN molecule to within $4.5 \AA$ at least once during the simulation. The remaining water molecules are hidden from view, so as not to obscure the solute molecule.)

By cross-referencing with the results of the gas-phase simulations, we find that polar solvation has a decisive influence on the rate and incidence of the formation of the $\mathrm{S}_{1}$-TICT structure. In the absence of the stabilizing effect of the polar solvent, the $\mathrm{S}_{1}$-TICT structure is not populated to a significant extent, at least not on the time scale of our simulations. On the other hand, polar solvation causes a rapid shift of the excited-state population into the $\mathrm{S}_{1}$-TICT structure. The $\mathrm{S}_{1}$-RICT structure is conspicuous by its absence - the molecule never adopts a geometry with a bent nitrile group, not even for a short period of time. Hence, the simulation results are clearly incompatible with the kinetic models II and III discussed previously in the Background section. On the other hand, model I gives a good match with the sequence of events that is observed in the simulations, provided that the ICT structure featuring in that model can be identified with the TICT structure. Accordingly, in what follows we interpret the simulation results in terms of kinetic model I.

To the best of our knowledge, no data is available in the literature on the kinetics of the dual fluorescence of DMABN in aqueous solution, which would be the most direct comparison to our simulation model. For this reason, we refer instead to the case of DMABN in acetonitrile, a polar organic solvent. According to Druzhinin and coworkers, ${ }^{28}$ the rate constant for the conversion of the LE structure into the ICT structure in acetonitrile at room temperature is $k_{a}=2.49 \times 10^{11} \mathrm{~s}^{-1}$, which corresponds to a LE lifetime of $1 / k_{a}=4.02 \mathrm{ps}$. 
Meanwhile, in the simulations of DMABN in the water droplet, an exponential fit to the population of the TICT structure (i.e., the combined population of the $60-90^{\circ}$ and 90-120 bins of $\tau$ ) yields a value of $k_{a}=7.5 \times 10^{11} \mathrm{~s}^{-1}$ (or, equivalently, a LE lifetime of $\left.1 / k_{a}=1.3 \mathrm{ps}\right)$. Thus, the rate constant obtained from the simulations is higher by a factor of roughly 3 than the experimental value. This discrepancy may be partially attributable to the fact that the experimental and simulated values refer to different solvents. There is also another possibility, however: in the molecular dynamics simulations, there may occur a leakage of zero-point energy from the fast vibrational modes of the DMABN molecule into the slow modes, including the intramolecular rotation mode. This effect may potentially increase the rate and incidence of intramolecular rotation.

\subsection{Time-Resolved Fluorescence Spectra}

We are now prepared to examine the TRF spectra resulting from the simulated relaxation dynamics of DMABN in the gas phase and in the water droplet, which are plotted in Figure 10 (a) and (b), respectively. We begin with the case of DMABN in the gas phase. At $t=0$, immediately after the initial photoexcitation, the simulated spectrum shows a sharp, intense feature in the energy range from around 4.5 to $5.0 \mathrm{eV}$, which is marked with an asterisk in Figure 10 (a). This feature arises from emission from the $\mathrm{S}_{2}$ state, and is rapidly decaying in intensity as the excited-state population undergoes internal conversion from the $S_{2}$ state to the $S_{1}$ state. Simultaneously, a weaker, broad emission band appears in the energy range from around 3.5 to $4.3 \mathrm{eV}$. This latter band can be identified as the normal fluorescence band of DMABN under gas-phase conditions, and originates from the $\mathrm{S}_{1}$ state of trajectories trapped in the potential energy well around the $\mathrm{S}_{1}$-LE minimum.

As mentioned previously in Section 3.3, the simulated trajectories continuously underwent brief excursions from the $S_{1}$ state into the $S_{2}$ state. As a consequence, the fluorescence signal of the $\mathrm{S}_{2}$ state does not disappear completely, but remains as a weak second band in the energy range from around 4.3 to $4.8 \mathrm{eV}$. (NB this effect should not be confused with dual 
fluorescence.) This second band is not present in the experimentally-observed fluorescence spectrum of DMABN vapor, ${ }^{73}$ which means that it is a simulation artifact. Presumably, the FSSH simulation overestimates the residual population of the $\mathrm{S}_{2}$ state, which leads to the emergence of the spurious high-energy fluorescence band in the simulated spectrum.

The effect of polar solvation on the development of the TRF spectrum can be seen in Figure 10 (b). Immediately after the initial photoexcitation, the spectrum consists of a sharp feature in the energy range around 4.4 to $4.9 \mathrm{eV}$, which is marked with an asterisk in Figure 10 (b). This short-lived signal is analogous to the one seen in the gas phase, and likewise originates from the $\mathrm{S}_{2}$ state. Within a few tens of femtoseconds, it gives way to a broad, moderately intense band in the range from around 3.2 to $4.2 \mathrm{eV}$. This latter band corresponds to the normal fluorescence band of DMABN in solution.

From around $t=400 \mathrm{fs}$, the intensity of the normal band gradually diminishes. At the same time, a new emission band emerges as a shoulder of the former band, in the energy range from around 1.5 to $3.0 \mathrm{eV}$. Clearly, this low-energy fluorescence band is the anomalous band, and it arises from those simulated trajectories that have reached the $\mathrm{S}_{1}$-TICT structure.

By way of comparison with experimental data, the steady-state fluorescence spectrum of DMABN in aqueous solution was recorded by Saigusa and coworkers. ${ }^{74}$ It consists of two slightly overlapping bands with well-separated maxima: the normal band shows a maximum at an energy of around $3.4 \mathrm{eV}$, while the anomalous fluorescence band peaks at around $2.4 \mathrm{eV}$. In contrast, in the simulated spectrum the normal and anomalous bands are somewhat indistinct. This may be partly due to the choppy appearance of the spectrum, which is a finite sampling artifact, resulting from the spectrum having been calculated on the basis of only 50 simulated trajectories. This point aside, the positions of the normal and anomalous fluorescence bands in the simulated spectrum are in reasonably good agreement with experiment. 
Figure 10: Simulated time-resolved fluorescence spectra of DMABN (a) in the gas phase and (b) in the 500-molecule water droplet. Emission intensity, in arbitrary units, is indicated with the use of color, and both spectra are normalized to the same intensity scale. The sharp, short-lived features marked with an asterisk originate from the $\mathrm{S}_{2}$ state.

(a) gas phase

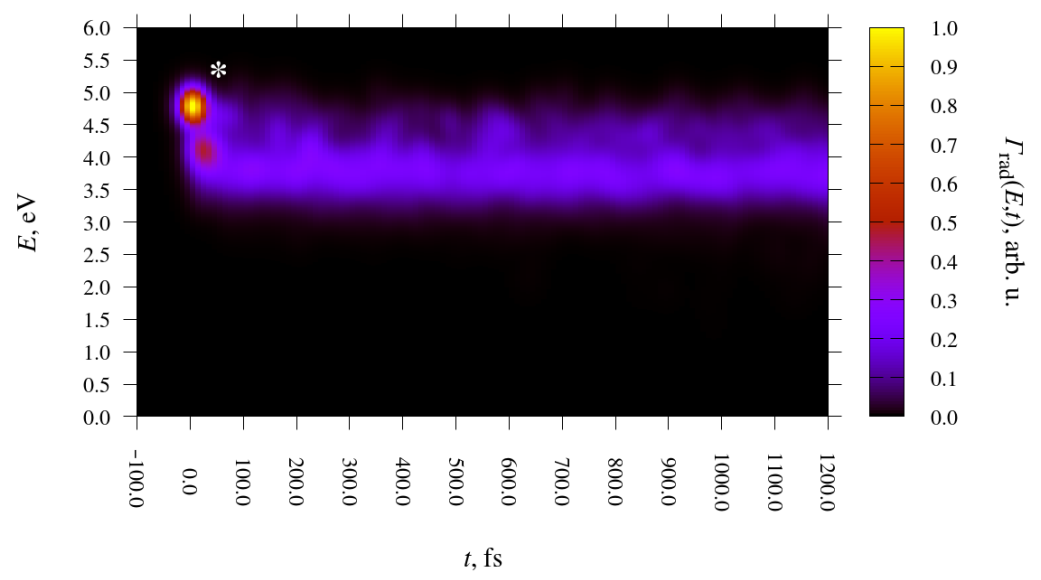

(b) water droplet

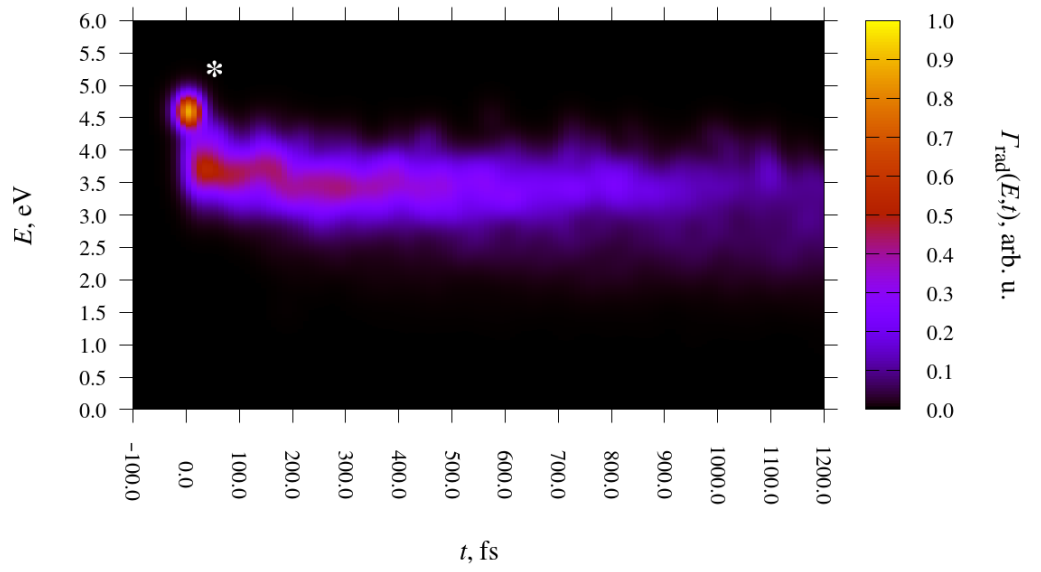

\subsection{Mechanism of Dual Fluorescence}

The mechanism of dual fluorescence of DMABN that emerges from the present simulations is shown schematically in Figure 11. The initial photoexcitation predominantly populates the $\mathrm{S}_{2}\left({ }^{1} \mathrm{~L}_{\mathrm{a}}\right)$ state. Within a few tens of femtoseconds, the system undergoes internal conversion into the $\mathrm{S}_{1}\left({ }^{1} \mathrm{~L}_{\mathrm{b}}\right)$ state. In the nonpolar environment of the gas phase, the vast majority of the excited-state population subsequently becomes trapped in the potential energy well of the $\mathrm{S}_{1}$-LE structure. This latter structure is responsible for the normal fluorescence band of 
Figure 11: Schematic illustration of the mechanism of dual fluorescence of DMABN in polar solution, as predicted by the present simulation model.

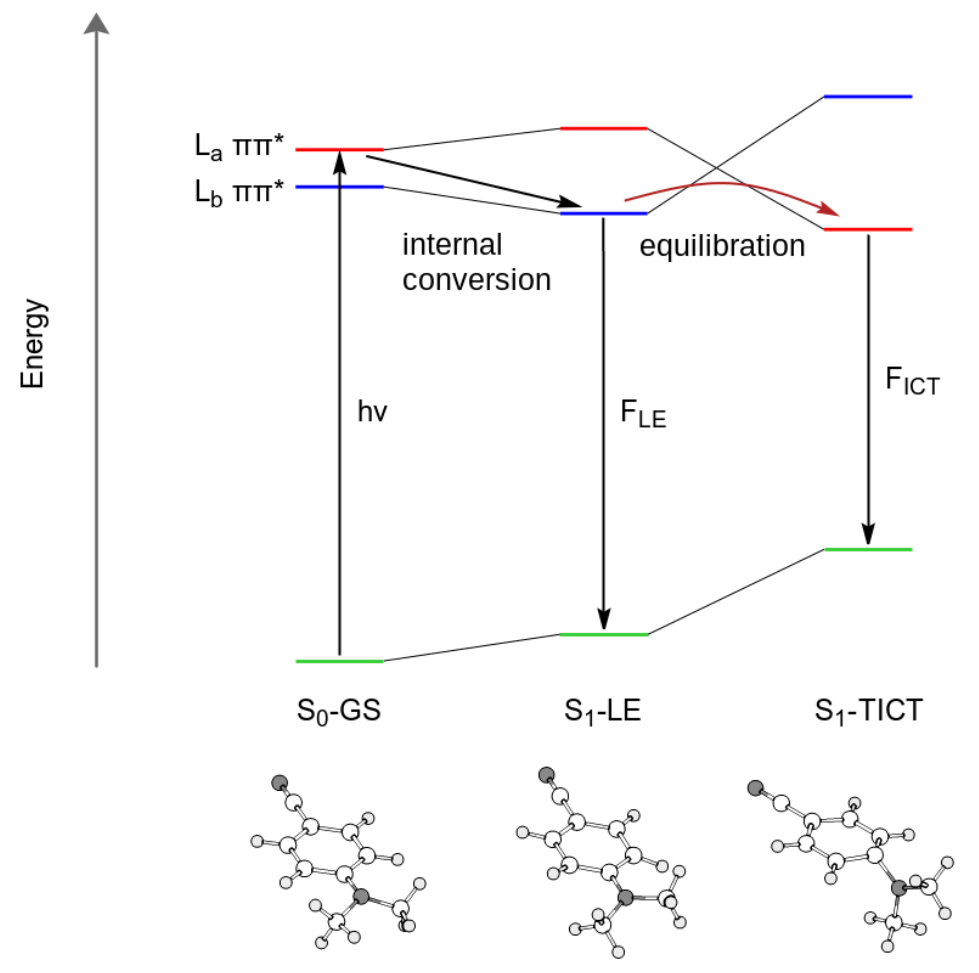

\section{DMABN.}

In the water nanodroplet, the situation is dramatically different. The excited-state population gradually escapes from the potential energy well around the $\mathrm{S}_{1}$-LE minimum, and eventually reaches the $\mathrm{S}_{1}$-TICT minimum. Subsequently, emission from the $\mathrm{S}_{1}$-TICT structure gives rise to the anomalous fluorescence band. The overall mechanism is compatible with kinetic model I proposed by Zachariasse et al. ${ }^{28,29}$ provided that the ICT structure appearing in that model can be identified with the $\mathrm{S}_{1}$-TICT structure. 


\section{Conclusions}

In this study, we applied nonadiabatic MD simulations in order to test several models of the excited-state relaxation process of DMABN, an archetypal system for dual fluorescence. The simulation results unambiguously point towards the TICT structure as responsible for the anomalous fluorescence band. There is no evidence for the involvement of other ICT structures, either as fluorescent species, or short-lived reaction intermediates. Moreover, the simulations show that the LE structure is converted directly into the TICT structure through a rotation of the dimethylamino group. The bending of the nitrile group is not required to facilitate the excited-state isomerization process.

On the methodological side, our findings showcase the ability of spin-component scaling (SCS) techniques (of which the SOS variant is a special case) to control the performance of $\mathrm{ADC}(2)$ for excited-state PESs. In the case of DMABN, the imposition of the SOS procedure affords the correct energy ordering of the $\mathrm{S}_{1}$-LE and $\mathrm{S}_{1}$-TICT structures, which is a prerequisite for a realistic picture of the dual fluorescence process. Encouragingly,

some earlier studies ${ }^{75-77}$ have likewise reported that SCS improves the accuracy of methods such as $\mathrm{CC} 2$ and $\mathrm{ADC}(2)$ for some systems where the unscaled methods perform poorly. Thus, the SCS approach may represent a general strategy to improve the reliability of these methods for applications in photochemistry.

\section{- Associated Content}

\section{Supporting Information}

Details of computational methods, structure of the $\mathrm{S}_{2} / \mathrm{S}_{1}$ conical intersection of DMABN, transition state for isomerization from $\mathrm{S}_{1}$-LE to $\mathrm{S}_{1}$-TICT, additional plots of time-resolved fluorescence spectra, and molecular geometries (PDF). Animations of simulated trajectories (MP4). 


\section{a Author Information}

\section{Corresponding Author}

*E-mail: m.kochman@ucl.ac.uk

Phone: +49 (0)16093180173

*E-mail: bodur@ifm.liu.se

Phone: +46 (0)13-28-24-97

\section{ORCID}

Michał A. Kochman: 0000-0003-2552-9464

Bo Durbeej: 0000-0001-5847-1196

\section{Notes}

The authors declare no competing financial interest.

\section{- Acknowledgements}

The authors are indebted to Dr Johan Raber and Mr Mats Kronberg for invaluable technical assistance. We gratefully acknowledge financial support from Stiftelsen Olle Engkvist Byggmästare (grant 184-568), from Wenner-Gren Stiftelserna (grant UPD2018-0102), and from Linköping University. We would also like to thank the National Supercomputer Center (NSC) in Linköping, Sweden, for their generous allotment of computer time. 


\section{口 References}

(1) Lippert, E.; Lüder, W.; Moll, F.; Nägele, W.; Boos, H.; Prigge, H.; Seibold-Blankenstein, I. Umwandlung von Elektronenanregungsenergie. Angew. Chem. 1961, 73, 695-706. DOI: 10.1002/ange.19610732103

(2) Lippert, E.; Lüder, W.; Boos, H. Fluoreszcenzspektrum und Franck-Condon-Prinzip in Lösungen Aromatischer Verbindungen. In Advances in Molecular Spectroscopy; Mangini, A., Ed.; MacMillan: New York, 1962; Vol. 1; pp 443-457. DOI: 10.1016/B978-1-4832-1332-3.50070-6

(3) Platt, J. R. Classification of Spectra of Cata-Condensed Hydrocarbons. J. Chem. Phys. 1949, 17, 484-495. DOI: 10.1063/1.1747293

(4) Kasha, M. Characterization of Electronic Transitions in Complex Molecules. Discuss. Faraday Soc. 1950, 9, 14-19. DOI: 10.1039/DF9500900014

(5) Rettig, W. Charge Separation in Excited States of Decoupled Systems-TICT Compounds and Implications Regarding the Development of New Laser Dyes and the Primary Process of Vision and Photosynthesis. Angew. Chem. Int. Ed. Engl. 1986, 25, 971-988. DOI: 10.1002/anie.198609711

(6) Rettig, W. Photoinduced Charge Separation via Twisted Intramolecular Charge Transfer States. In Electron Transfer I. Topics in Current Chemistry; Mattay, J., Ed.; Springer: Berlin, Heidelberg, 1994; Vol. 169; pp 253-299. DOI: $10.1007 / 3-540-57565-0 \_78$

(7) Grabowski, Z. R.; Rotkiewicz, K.; Rettig, W. Structural Changes Accompanying Intramolecular Electron Transfer: Focus on Twisted Intramolecular Charge-Transfer States and Structures. Chem. Rev. 2003, 103, 3899-4031. DOI: 10.1021/cr9407451 
(8) Misra, R.; Bhattacharyya, S. P. Intramolecular Charge Transfer. Theory and Applications. Wiley-VCH Verlag: Weinheim, Germany, 2018.

(9) Rotkiewicz, K.; Grellmann, K. H.; Grabowski, Z. R. Reinterpretation of the Anomalous Fluorescence of $p$-N,N-Dimethylamino-benzonitrile. Chem. Phys. Lett. 1973, 19, 315-318. DOI: 10.1016/0009-2614(73)80367-7

(10) Serrano-Andrés, L.; Merchán, M.; Roos, B. O.; Lindh, R. Theoretical Study of the Internal Charge Transfer in Aminobenzonitriles. J. Am. Chem. Soc. 1995, 117, 3189-3204. DOI: 10.1021/ja00116a024

(11) Köhn, A.; Hättig, C. On the Nature of the Low-Lying Singlet States of 4-(Dimethyl-amino)benzonitrile. J. Am. Chem. Soc. 2004, 126, 7399-7410. DOI: $10.1021 / \mathrm{ja} 0490572$

(12) Gómez, I.; Reguero, M.; Boggio-Pasqua, M.; Robb, M. A. Intramolecular Charge Transfer in 4-Aminobenzonitriles Does Not Necessarily Need the Twist. J. Am. Chem. Soc. 2005, 127, 7119-7129. DOI: 10.1021/ja042413w

(13) Grabowski, Z. R.; Rotkiewicz, K.; Siemiarczuk, A. Dual Fluorescence of Donor-Acceptor Molecules and the Twisted Intramolecular Charge Transfer (TICT) States. J. Lumin. 1979, 18/19, 420-424. DOI: 10.1016/0022-2313(79)90153-4

(14) Rotkiewicz, K.; Rubaszewska, W. Intramolecular Electron-Transfer Excited State in 6-Cyanobenzquinuclidine. Chem. Phys. Lett. 1980, 70, 444-448. DOI: $10.1016 / 0009-2614(80) 80101-1$

(15) Rotkiewicz, K.; Rubaszewska, W. Intramolecular Charge Transfer State and Unusual Fluorescence from an Upper Excited Singlet of a Nonplanar Derivative of $p$-Cyano- $N, N$-dimethylaniline. J. Lumin. 1982, 27, 221-230. DOI: $10.1016 / 0022-2313(82) 90023-0$ 
(16) Okada, T.; Uesugi, M.; Köhler, G.; Rechthaler, K.; Rotkiewicz, K.; Rettig, W.; Grabner, G. Time-Resolved Spectroscopy of DMABN and its Cage Derivatives 6-Cyanobenzquinuclidine (CBQ) and Benzquinuclidine (BQ). Chem. Phys. 1999, 241, 327-337. DOI: 10.1016/S0301-0104(98)00411-X

(17) Zachariasse, K. A.; Grobys, M.; von den Haar, Th.; Hebecker, A.; Il'ichev, Yu. V.; Jiang, Y.-B.; Morawski, O.; Kühnle, W. Intramolecular Charge Transfer in the Excited State. Kinetics and Configurational Change. J. Photochem. Photobiol. A 1996, 102, 59-70. DOI: 10.1016/S1010-6030(96)04368-7

(18) Zachariasse, K. A.; Grobys, M.; von den Haar, Th.; Hebecker, A.; Il'ichev, Yu. V.; Jiang, Y.-B.; Morawski, O.; Rückert, I.; Kühnle, W. Photoinduced Intramolecular Charge Transfer and Internal Conversion in Molecules with a Small Energy Gap between $\mathrm{S}_{1}$ and $\mathrm{S}_{2}$. Dynamics and Structure. J. Photochem. Photobiol. A 1997, 105, 373-383. DOI: 10.1016/S1010-6030(96)04601-1

(19) Zachariasse, K. A.; Druzhinin, S. I.; Bosch, W.; Machinek, R. Intramolecular Charge Transfer with the Planarized 4-Aminobenzonitrile 1-tert-Butyl-6-cyano-1,2,3,4-tetrahydroquinoline (NTC6). J. Am. Chem. Soc. 2004, 126, 1705-1715. DOI: 10.1021/ja037544w

(20) Hättig, C.; Hellweg, A.; Köhn, A. Intramolecular Charge-Transfer Mechanism in Quinolidines: The Role of the Amino Twist Angle. J. Am. Chem. Soc. 2006, 128, 15672-15682. DOI: $10.1021 /$ ja0642010

(21) Sobolewski, A. L.; Domcke, W. Charge Transfer in Aminobenzonitriles: Do They Twist? Chem. Phys. Lett. 1996, 250, 428-436. DOI: 10.1016/0009-2614(96)00014-0

(22) Sobolewski, A. L.; Domcke, W. Promotion of Intramolecular Charge Transfer in Dimethylamino Derivatives: Twisting Versus Acceptor-Group Rehybridization. Chem. Phys. Lett. 1996, 259, 119-127. DOI: 10.1016/0009-2614(96)00724-5 
(23) Foresman, J. B.; Head-Gordon, M.; Pople, J. A.; Frisch, M. J. Toward a Systematic Molecular Orbital Theory for Excited States. J. Phys. Chem. 1992, 96, 135-149. DOI: $10.1021 / \mathrm{j} 100180 \mathrm{a} 030$

(24) Andersson, K.; Malmqvist, P.-A.; Roos, B. O.; Sadlej, A.; Wolinski, K. Second-Order Perturbation Theory with a CASSCF Reference Function. J. Phys. Chem. 1990, 94, 5483-5488. DOI: $10.1021 / \mathrm{j} 100377 \mathrm{a} 012$

(25) Andersson, K.; Malmqvist, P.-A.; Roos, B. O. Second-Order Perturbation Theory with a Complete Active Space Self-Consistent Field Reference Function. J. Chem. Phys. 1992, 96, 1218-1226. DOI: 10.1063/1.462209

(26) Sobolewski, A. L.; Sudholt, W.; Domcke, W. Ab Initio Investigation of Reaction Pathways for Intramolecular Charge Transfer in Dimethylanilino Derivatives. J. Phys. Chem. A 1998, 102, 2716-2722. DOI: 10.1021/jp980435x

(27) Sudholt, W.; Sobolewski, A. L.; Domcke, W. Ab Initio Study of the Amino Group Twisting and Wagging Reaction Paths in the Intramolecular Charge Transfer of 4-(N,N-Dimethylamino)benzonitrile. Chem. Phys. 1999, 240, 9-18. DOI: 10.1016/S0301-0104(98)00368-1

(28) Druzhinin, S. I.; Ernsting, N. P.; Kovalenko, S. A.; Lustres, L. P.; Senyushkina, T. A.; Zachariasse, K. A. Dynamics of Ultrafast Intramolecular Charge Transfer with 4-(Dimethylamino)benzonitrile in Acetonitrile. J. Phys. Chem. A 2006, 110, 2955-2969. DOI: 10.1021/jp054496o

(29) Zachariasse, K. A.; Druzhinin, S. I.; Kovalenko, S. A.; Senyushkina, T. Intramolecular Charge Transfer of 4-(Dimethylamino)benzonitrile Probed by Time-Resolved Fluorescence and Transient Absorption: No Evidence for two ICT States and a $\pi \sigma^{*}$ Reaction Intermediate. J. Chem. Phys. 2009, 131, 224313. DOI: 10.1063/1.3270165 
(30) Fujiwara, T.; Zgierski, M. Z.; Lim, E. C. The Role of the $\pi \sigma^{*}$ State in the Intramolecular Charge Transfer of 4-(Dimethylamino)benzonitrile. Phys. Chem. Chem. Phys. 2011, 13, 6779-6783.

(31) Zgierski, M. Z.; Lim, E. C. The Role of $\pi \sigma^{*}$ State in Intramolecular Electron-Transfer Dynamics of 4-Dimethylaminobenzonitrile and Related Molecules. J. Chem. Phys. 2004, 121, 2462-2465. DOI: 10.1063/1.1780154

(32) Zgierski, M. Z.; Lim, E. C. Electronic and Vibrational Spectra of the Low-Lying $\pi \sigma^{*}$ State of 4-dimethylaminobenzonitrile: Comparison of Theoretical Predictions with Experiment. J. Chem. Phys. 2005, 122, 111103. DOI: 10.1063/1.1889431

(33) Galván, I. F.; Martín, M. E.; Aguilar, M. A. Theoretical Study of the Dual Fluorescence of 4-(N,N-Dimethylamino)benzonitrile in Solution. J. Chem. Theory Comput. 2010, 6, 2445-2454. DOI: 10.1021/ct9006713

(34) Galván, I. F.; Martín, M. E.; Aguilar, M. A. On the Absorption Properties of the Excited States of DMABN. Chem. Phys. Lett. 2010, 499, 100-102. DOI: $10.1016 /$ j.cplett.2010.09.029

(35) Coto, P. B.; Serrano-Andrés, L.; Gustavsson, T.; Fujiwara, T.; Lim, E. C. Intramolecular Charge Transfer and Dual Fluorescence of 4-(Dimethylamino)benzonitrile: Ultrafast Branching Followed by a Two-Fold Decay Mechanism. Phys. Chem. Chem. Phys. 2011, 13, 15182-15188. DOI: $10.1039 / \mathrm{c} 1 \mathrm{cp} 21089 \mathrm{k}$

(36) Fonseca, T.; Kim, H. J.; Hynes, J. T. Twisted Intramolecular Charge Transfer Dynamics in Polar Solvents. J. Photochem. Photobiol. A: Chem. 1994, 82, 67-79. DOI: 10.1016/1010-6030(94)02018-3

(37) Fonseca, T.; Kim, H. J.; Hynes, J. T. Dynamics of Twisted Intramolecular 
Charge Transfer Complexes in Polar Solvents. J. Mol. Liq. 1994, 60, 161-200. DOI: $10.1016 / 0167-7322(94) 00744-6$

(38) Kim, H.; Hynes, J. T. Excited State Intramolecular Charge Transfer Rates of $p$-Dimethylaminobenzonitrile (DMABN) in Solution: a Two-Dimensional Dynamics Perspective. J. Photochem. Photobiol. A: Chem. 1997, 105, 337-343. DOI: 10.1016/S1010-6030(96)04543-1

(39) Dorairaj, S.; Kim, H. J. Excited-State Charge Transfer Dynamics of p-Dimethylaminobenzonitrile in Quadrupolar Solvents. J. Phys. Chem. A 2002, 106, 2322-2327. DOI: 10.1021/jp012991o

(40) Perveaux, A.; Castro, P. J.; Lauvergnat, D.; Reguero, M.; Lasorne, B. Intramolecular Charge Transfer in 4-Aminobenzonitrile Does Not Need the Twist and May Not Need the Bend. J. Phys. Chem. Lett. 2015, 6, 1316-1320. DOI: 10.1021/acs.jpclett.5b00162

(41) Du, L.; Lan, Z. An On-the-Fly Surface-Hopping Program JADE for Nonadiabatic Molecular Dynamics of Polyatomic Systems: Implementation and Applications. J. Chem. Theory Comput. 2015, 11, 1360-1374. DOI: 10.1021/ct501106d

(42) Castro, P. J.; Perveaux, A.; Lauvergnat, D.; Reguero, M.; Lasorne, B. Ultrafast Internal Conversion in 4-Aminobenzonitrile Occurs Sequentially Along the Seam. Chem. Phys. 2018, 509, 30-36. DOI: 10.1016/j.chemphys.2018.03.013

(43) Kochman, M. A.; Tajti, A.; Morrison, C. A.; Miller, R. J. D. Early Events in the Nonadiabatic Relaxation Dynamics of 4-( $N, N$-Dimethylamino)benzonitrile. J. Chem. Theory Comput. 2015, 11, 1118-1128. DOI: 10.1021/ct5010609

(44) Curchod, B. F. E.; Sisto, A.; Martínez, T. J. Ab Initio Multiple Spawning Photochemical Dynamics of DMABN Using GPUs. J. Phys. Chem. A 2017, 121, 265-276. DOI: 10.1021/acs.jpca.6b09962 
(45) Medders, G. R.; Alguire, E. C.; Jain, A.; Subotnik, J. E. Ultrafast Electronic Relaxation through a Conical Intersection: Nonadiabatic Dynamics Disentangled through an Oscillator Strength-Based Diabatization Framework. J. Phys. Chem. A 2017, 121, 1425-1434. DOI: 10.1021/acs.jpca.6b12120

(46) Trofimov, A. B.; Schirmer, J. An Efficient Polarization Propagator Approach to Valence Electron Excitation Spectra. J. Phys. B: At., Mol. Opt. Phys. 1995, 28, 2299-2324. DOI: 10.1088/0953-4075/28/12/003.

(47) Hättig, C. Structure Optimizations for Excited States with Correlated Second-Order Methods: $\mathrm{CC} 2$ and $\mathrm{ADC}(2)$. In Advances in Quantum Chemistry; Jensen, H. J. A.., Ed.; Academic Press: New York, 2005; Vol. 50; pp 37-60. DOI: $10.1016 / \mathrm{S} 0065-3276(05) 50003-0$.

(48) Jung, Y.; Lochan, R. C.; Dutoi, A. D.; Head-Gordon, M. Scaled Opposite-Spin Second Order Møller-Plesset Correlation Energy: An Economical Electronic Structure Method. J. Chem. Phys. 2004, 121, 9793-9802. DOI: 10.1063/1.1809602

(49) Winter, N. O. C.; Hättig, C. Scaled Opposite-Spin CC2 for Ground and Excited States with Fourth Order Scaling Computational Costs. J. Chem. Phys. 2011, 134, 184101. DOI: $10.1063 / 1.3584177$

(50) TURBOMOLE V6.3.1 2011, a development of University of Karlsruhe and Forschungszentrum Karlsruhe GmbH, 1989-2007, TURBOMOLE GmbH, since 2007; available from http://www.turbomole.com

(51) Haase, F.; Ahlrichs, R. Semi-direct MP2 Gradient Evaluation on Workstation Computers: The MPGRAD Program. J. Comp. Chem., 1993, 14, 907-912, DOI: $10.1002 /$ jcc.540140805

(52) Weigend, F.; Häser, M. RI-MP2: First Derivatives and Global Consistency. Theor. Chem. Acc., 1997, 97, 331-340, DOI: 10.1007/s002140050269 
(53) Hättig, C.; Weigend, F. CC2 Excitation Energy Calculations on Large Molecules Using the Resolution of the Identity Approximation. J. Chem. Phys., 2000, 113, 5154-5161, DOI: $10.1063 / 1.1290013$

(54) Köhn, A.; Hättig, C. Analytic Gradients for Excited States in the Coupled-Cluster Model CC2 Employing the Resolution-of-the-Identity Approximation. J. Chem. Phys., 2003, 119, 5021-5036, DOI: 10.1063/1.1597635

(55) Dunning Jr, T. H. Gaussian Basis Sets for Use in Correlated Molecular Calculations. I. The Atoms Boron Through Neon and Hydrogen. J. Chem. Phys. 1989, 90, 1007-1023. DOI: 10.1063/1.456153

(56) Weigend, F.; Köhn, A.; Hättig, C. Efficient Use of the Correlation Consistent Basis Sets in Resolution of the Identity MP2 Calculations. J. Chem. Phys., 2002, 116, 3175-3183, DOI: 10.1063/1.1445115

(57) Lin, H.; Truhlar, D. G. QM/MM: What Have We Learned, Where Are We, and Where Do We Go From Here? Theor. Chem. Acc. 2007, 117, 185-199. DOI: $10.1007 / \mathrm{s} 00214-006-0143-\mathrm{z}$

(58) Senn, H. M.; Thiel, W. QM/MM Methods for Biological Systems. Top. Curr. Chem. 2007, 268, 173-290. DOI: 10.1007/128_2006_084

(59) Schmitt, U. W.; Voth, G. A. The Computer Simulation of Proton Transport in Water. J. Chem. Phys. 1999, 111, 9361-9381. DOI: 10.1063/1.480032

(60) Tully, J. C.; Preston, R. K. Trajectory Surface Hopping Approach to Nonadiabatic Molecular Collisions: The Reaction of $\mathrm{H}^{+}$with $\mathrm{D}_{2}$. J. Chem. Phys. 1971, 55, 562-572. DOI: $10.1063 / 1.1675788$

(61) Tully, J. C. Molecular Dynamics with Electronic Transitions. J. Chem. Phys. 1990, 93, 1061-1071. DOI: 10.1063/1.459170 
(62) Hammes-Schiffer, S.; Tully, J. C. Proton Transfer in Solution: Molecular Dynamics With Quantum Transitions. J. Chem. Phys. 1994, 101, 4657-4667. DOI: $10.1063 / 1.467455$

(63) Doltsinis, N. L.; Marx, D. First Principles Molecular Dynamics Involving Excited States and Nonadiabatic Transitions. J. Theor. Comput. Chem. 2002, 1, 319-349. DOI: $10.1142 / \mathrm{S} 0219633602000257$

(64) Plasser, F.; Ruckenbauer, M.; Mai, S.; Oppel, M.; Marquetand, P.; González, L. Efficient and Flexible Computation of Many-Electron Wavefunction Overlaps. J. Chem. Theory Comput. 2016, 12, 1207-1219. DOI: 10.1021/acs.jctc.5b01148

(65) Plasser, F.; González, L. Communication: Unambiguous Comparison of Many-Electron Wavefunctions Through Their Overlaps. J. Chem. Phys. 2003, 145, 021103. DOI: $10.1063 / 1.4958462$

(66) Crespo-Otero, R.; Barbatti, M. Spectrum Simulation and Decomposition with Nuclear Ensemble: Formal Derivation and Application to Benzene, Furan and 2-Phenylfuran. Theor. Chem. Acc. 2012, 131, 1237. DOI: 10.1007/s00214-012-1237-4

(67) Christiansen, O.; Koch, H.; Jørgensen, P. Perturbative Triple Excitation Corrections to Coupled Cluster Singles and Doubles Excitation Energies. J. Chem. Phys. 1996, 105, 1451-1459. DOI: $10.1063 / 1.472007$

(68) Gómez, I.; Castro, P. J.; Reguero, M. Insight into the Mechanisms of Luminescence of Aminobenzonitrile and Dimethylaminobenzonitrile in Polar Solvents. An ab Initio Study. J. Phys. Chem. A 2015, 119, 1983-1995. DOI: 10.1021/acs.jpca.5b01421

(69) Georgieva, I.; Aquino, A. J. A.; Plasser, F.; Trendafilova, N.; Köhn, A.; Lischka, H. Intramolecular Charge-Transfer Excited-State Processes in 4-( $N, N$-Dimethylamino)benzonitrile: The Role of Twisting and the $\pi \sigma^{*}$ State. J. Phys. Chem. A 2015, 119, 6232-6243. DOI: 10.1021/acs.jpca.5b03282 
(70) Bowman, J. M.; Gazdy, B.; Sun, Q. A Method to Constrain Vibrational Energy in Quasiclassical Trajectory Calculations. J. Chem. Phys. 1989, 91, 2859-2862. DOI: $10.1063 / 1.456955$

(71) Guo, Y.; Thompson, D. L.; Sewell, T. D. Analysis of the Zero-Point Energy Problem in Classical Trajectory Simulations. J. Chem. Phys. 1996, 104, 576-582. DOI: $10.1063 / 1.470853$

(72) Martínez-Fernández, L.; Corral, I.; Granucci, G.; Persico, M. Competing Ultrafast Intersystem Crossing and Internal Conversion: A Time Resolved Picture for the Deactivation of 6-Thioguanine. Chem. Sci. 2014, 5, 1336-1347. DOI: $10.1039 / \mathrm{c} 3 \mathrm{sc} 52856 \mathrm{a}$

(73) Druzhinin, S. I.; Mayer, P.; Stalke, D.; von Bülow, R.; Noltemeyer, M.; Zachariasse, K. A. Intramolecular Charge Transfer with 1-tert-Butyl-6-cyano-1,2,3,4-tetrahydroquinoline (NTC6) and Other Aminobenzonitriles. A Comparison of Experimental Vapor Phase Spectra and Crystal Structures with Calculations. J. Am. Chem. Soc. 2010, 132, 7730-7744. DOI: $10.1021 / \mathrm{ja} 101336 \mathrm{n}$

(74) Saigusa, H.; Iwase, E.; Nishimura, M. Structure and Intramolecular Charge-Transfer Dynamics of $p$-(Dimethylamino)benzonitrile in Solvent Clusters. J. Phys. Chem. A 2003, 107, 4989-4998. DOI: 10.1021/jp030037g

(75) Aquino, A. J. A.; Borges Jr, I.; Nieman, R.; Köhn, A.; Lischka, H. Intermolecular Interactions and Charge Transfer Transitions in Aromatic Hydrocarbon-Tetracyanoethylene Complexes. Phys. Chem. Chem. Phys. 2014, 16, 20586-20597. DOI: $10.1039 / \mathrm{c} 4 \mathrm{cp} 02900 \mathrm{c}$

(76) Tajti, A.; Szalay, P. G. Accuracy of Spin-Component-Scaled CC2 Excitation Energies 
and Potential Energy Surfaces. J. Chem. Theory Comput. 2019, 15, 5523-5531. DOI: $10.1021 /$ acs.jctc.9b00676

(77) Tajti, A.; Tulipán, L.; Szalay, P. G. Accuracy of Spin-Component Scaled ADC(2) Excitation Energies and Potential Energy Surfaces. J. Chem. Theory Comput. 2020, 16, 468-474. DOI: 10.1021/acs.jctc.9b01065 


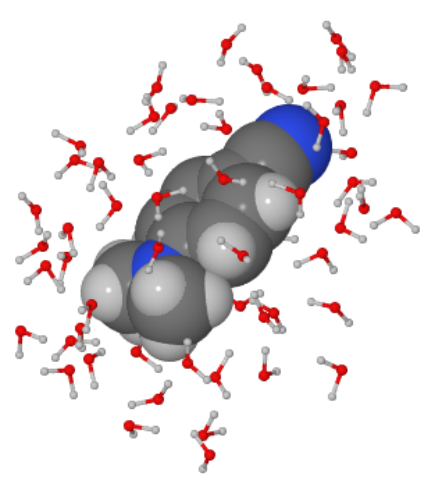

$t=375 \mathrm{fs}$

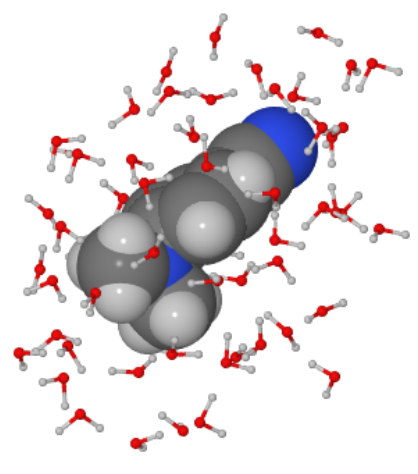

$t=625 \mathrm{fs}$

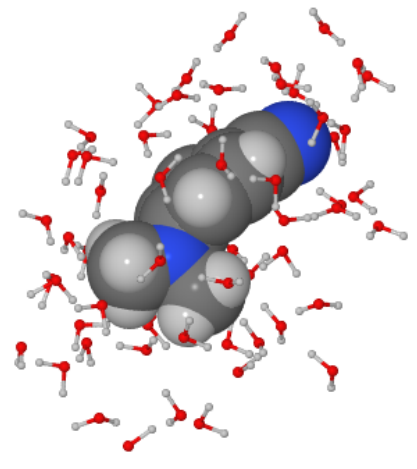

$t=750 \mathrm{fs}$ 


\title{
Supporting Information for Simulating the Nonadiabatic Relaxation Dynamics of 4-( $N, N$-Dimethylamino)benzonitrile (DMABN) in Polar Solution
}

\author{
Michał Andrzej Kochman, ${ }^{\dagger}$ Bo Durbeej ${ }^{\dagger}$
}

\begin{abstract}
$\dagger$ Division of Theoretical Chemistry, Department of Physics, Chemistry and Biology (IFM), Linköping University, 58183 Linköping, Sweden.

$\ddagger$ Department of Chemistry, University College London (UCL), 20 Gordon Street, London WC1H 0A, United Kingdom

e-mail: m.kochman@ucl.ac.uk, bodur@ifm.liu.se
\end{abstract}

\section{Contents}

S1 Details of Computational Methods $\quad$ S2

S1.1 QM/MM Calculations . . . . . . . . . . . . . . S2

S1.2 Initial Conditions . . . . . . . . . . . . . . . . . . . S4

S1.3 Nonadiabatic Molecular Dynamics . . . . . . . . . . . . . . . . S7

S1.4 Choice of Electronic Structure Method . . . . . . . . . . . . . . S8

S1.5 Spin-Opposite Scaling and Electronic Structure . . . . . . . . . . . . S11

S1.6 ADC(3) Calculation of Relative Energies . . . . . . . . . . . . . . . . . S16

$\begin{array}{lr}\text { S2 Electronic Excitation Spectrum of DMABN } & \text { S18 }\end{array}$

S3 Structure of the $\mathrm{S}_{2} / \mathrm{S}_{1}$ Conical Intersection of DMABN $\quad$ S20

S4 Transition State for Isomerization from $\mathrm{S}_{1}$-LE to $\mathrm{S}_{1}$-TICT $\quad$ S23

S5 Additional Plots of Time-Resolved Fluorescence Spectra $\quad$ S24

$\begin{array}{lr}\text { S6 Molecular Geometries } & \text { S26 }\end{array}$

$\begin{array}{lr}\text { References } & \text { S32 }\end{array}$ 


\section{S1 Details of Computational Methods}

\section{S1.1 QM/MM Calculations}

The potential energy surfaces (PESs) of the system were constructed with the use of the hybrid quantum mechanics/molecular mechanics ${ }^{1-3}(\mathrm{QM} / \mathrm{MM})$ method. Within the framework of that technique, the system under study (denoted $\mathbb{S}$ ) is partitioned into two subsystems which are treated at different levels of approximation. The electronic structure of the so-called inner subsystem (II) is explicitly included in the simulation, and is calculated with the use of a quantum-mechanical (QM) method. The outer subsystem $(\mathbb{O})$, in turn, is described with a molecular mechanics (MM) force field.

In the present case, the natural choice of $\mathrm{QM} / \mathrm{MM}$ partitioning is that the DMABN molecule is included in the inner subsystem, and the surrounding water molecules comprise the outer subsystem (see Figure 4 in the main body of this paper). Because periodic boundary conditions are not available in Turbomole, in our simulations the solution phase was represented by placing the solute molecule at the center of a water droplet containing 500 water molecules.

We elected to employ the additive variant ${ }^{3}$ of the $\mathrm{QM} / \mathrm{MM}$ scheme. In this approach, the potential energy function $\left(E_{\mathrm{QM} / \mathrm{MM}}(\mathbb{S})\right)$ of the system is broken down as follows:

$$
E_{\mathrm{QM} / \mathrm{MM}}(\mathbb{S})=E_{\mathrm{QM}}(\mathbb{I})+E_{\mathrm{MM}}(\mathbb{O})+E_{\mathrm{QM}-\mathrm{MM}}(\mathbb{I}, \mathbb{O})
$$

Here, $E_{\mathrm{QM}}(\mathbb{I})$ represents the energy of the inner subsystem calculated at the QM level of theory. $E_{\mathrm{MM}}(\mathbb{O})$ is the energy of the outer subsystem, evaluated at the MM level of theory. Lastly, $E_{\mathrm{QM}-\mathrm{MM}}(\mathbb{I}, \mathbb{O})$ is the QM-MM coupling term, whose purpose is to account for the interactions between the inner and outer subsystems.

In the present implementation, the inner and outer subsystems interact via electrostatic and Van der Waals interactions. The electrostatic interactions are handled with the use of the electrostatic embedding scheme, ${ }^{3}$ whereby the QM calculation for the inner subsystem is performed in the presence of the charge distribution of the outer subsystem. In other words, the point charges of the MM force field are included in the QM Hamiltonian. This measure allows the electronic structure of the inner subsystem to adapt to the charge distribution of its environment. Because the electrostatic part of the interaction between the inner and outer subsystems is calculated during the QM calculation, it is subsumed into the term $E_{\mathrm{QM}}$, which becomes a function of $\mathbb{O}$ in addition to being a function of $\mathbb{I}$. As a consequence, equation 1 takes on a modified form:

$$
E_{\mathrm{QM} / \mathrm{MM}}(\mathbb{S})=E_{\mathrm{QM}}(\mathbb{I}, \mathbb{O})+E_{\mathrm{MM}}(\mathbb{O})+E_{\mathrm{QM}-\mathrm{MM}}(\mathbb{I}, \mathbb{O})
$$

The electronic structure of DMABN (more specfically, the term $E_{\mathrm{QM}}(\mathbb{I}, \mathbb{O})$ ) was evaluated at the SOS-ADC(2)/cc-pVDZ level of theory (see Section 1.4 below).

The energy of the solvent (namely, the term $E_{\mathrm{MM}}(\mathbb{O})$ ) was described with the use 
of the flexible TIP3P (TIP3P/Fs) water model as parameterized by Schmitt and Voth, ${ }^{4}$ whose functional form is as follows:

$$
\begin{aligned}
E_{\mathrm{MM}}(\mathbb{O})= & \sum_{i, j \in \mathbb{O}}^{\text {bonds }} \frac{1}{2} k_{i j}\left(r_{i j}-r_{i j}^{0}\right)^{2}+\sum_{i, j, k \in \mathbb{O}}^{\text {angles }} \frac{1}{2} k_{i j k}\left(\theta_{i j k}-\theta_{i j k}^{0}\right)^{2}+ \\
& +\sum_{i, j \in \mathbb{O}}^{\text {inter }}\left\{4 \varepsilon_{i j}\left[\left(\frac{\sigma_{i j}}{r_{i j}}\right)^{12}-\left(\frac{\sigma_{i j}}{r_{i j}}\right)^{6}\right]+\frac{q_{i} q_{j}}{r_{i j}}\right\}
\end{aligned}
$$

The first term on the right-hand side of equation 3 accounts for the stretching of $\mathrm{O}-\mathrm{H}$ bonds, the second term describes the bending of the water molecule (the angle between the two $\mathrm{O}-\mathrm{H}$ bonds), and the third term handles all water-water intermolecular interactions. The values of the interaction parameters are listed in Table S1.

The Van der Waals interactions between the inner and outer subsystems are retained in the QM-MM coupling term $\left(E_{\mathrm{QM}-\mathrm{MM}}(\mathbb{I}, \mathbb{O})\right)$, and are modeled with the 6-12 Lennard-Jones potential:

$$
E_{\mathrm{QM}-\mathrm{MM}}(\mathbb{I}, \mathbb{O})=\sum_{i \in \mathbb{I}}^{\text {atoms }} \sum_{j \in \mathbb{O}}^{\text {atoms }} 4 \varepsilon_{i j}\left[\left(\frac{\sigma_{i j}}{r_{i j}}\right)^{12}-\left(\frac{\sigma_{i j}}{r_{i j}}\right)^{6}\right]
$$

The van der Waals parameters for the atoms of DMABN were taken from the all-atom Optimized Potential for Liquid Simulations ${ }^{5}$ (OPLS/AA) force field. The assignment of atom types is shown in Figure S1. The carbon atoms comprising the phenyl ring were considered to have the "aromatic $\underline{\mathrm{C}}$ " atom type. Their hydrogen atoms were assigned the "aromatic $\underline{\mathrm{H}}-\mathrm{C}$ " atom type. The carbon and nitrogen atoms of the nitrile group were assigned the "benzonitrile $\underline{\mathrm{CN}}$ " and "benzonitrile $\mathrm{C} \underline{\mathrm{N}}$ " atom types, respectively. The nitrogen atom of the dimethylamino group was described with the "amine $\mathrm{R}_{3} \mathrm{~N}$ " atom type. Lastly, the "amine $\underline{\mathrm{CH}}_{3}-\mathrm{NR}_{2}$ " and "amine $\underline{\mathrm{H}}-\mathrm{C}-\mathrm{N}$ " atom types were used for the atoms comprising the two methyl groups.

Figure S1: The assignment of MM atom types to atoms of DMABN.
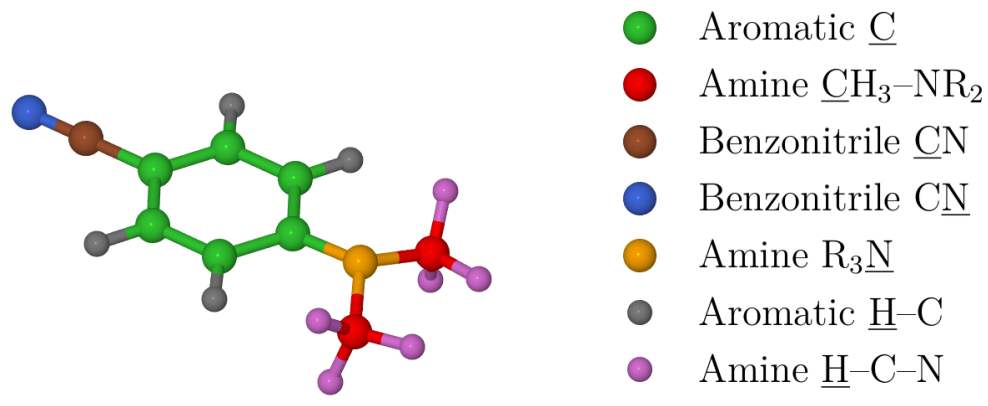
Table S1: Force field parameters used in the present study.

(a) Parameters for intermolecular interactions. ${ }^{4,5}$

\begin{tabular}{llll}
\hline Atom type $(i)$ & $\varepsilon_{i i}, \mathrm{kcal} / \mathrm{mol}$ & $\sigma_{i i}, \AA$ & $q, e$ \\
\hline Aromatic $\underline{\mathrm{C}}$ & 0.0700 & 3.5500 & \\
Amine $\underline{\mathrm{CH}_{3}-\mathrm{NR}_{2}}$ & 0.0660 & 3.5000 & \\
Benzonitrile $\underline{\mathrm{CN}}$ & 0.1500 & 3.6500 & \\
Benzonitrile $\underline{\mathrm{C}} \underline{\mathrm{N}}$ & 0.1700 & 3.2000 & \\
Amine $\mathrm{R}_{3} \underline{\mathrm{N}}$ & 0.1700 & 3.3000 & \\
Aromatic $\underline{\mathrm{H}}-\mathrm{C}$ & 0.0300 & 2.4200 & \\
Amine $\underline{\mathrm{H}}-\mathrm{C}-\mathrm{N}$ & 0.0150 & 2.5000 & \\
$\mathrm{O}$ (water) & 0.1522 & 3.1506 & -0.834 \\
$\mathrm{H}$ (water) & 0 & N/A & +0.417 \\
\hline
\end{tabular}

(b) Parameters for intramolecular interactions of TIP3P/Fs water. ${ }^{4}$

$$
\begin{array}{ll}
k_{\mathrm{OH}}=1059.162 \mathrm{kcal} \mathrm{mol}^{-1} \AA^{-2} & r_{\mathrm{OH}}^{0}=0.96 \AA \\
k_{\mathrm{HOH}}=68.087 \mathrm{kcal} \mathrm{mol}^{-1} \mathrm{rad}^{-2} & \theta_{\mathrm{HOH}}^{0}=104.5^{\circ}
\end{array}
$$

As is conventional in the OPLS/AA force field, the "mixed" interaction parameters $\sigma_{i j}$ and $\varepsilon_{i j}$ which appear in equation 4 were obtained with the use of geometric combining rules: $\sigma_{i j}=\left(\sigma_{i i} \sigma_{j j}\right)^{1 / 2}$ and $\varepsilon_{i j}=\left(\varepsilon_{i i} \varepsilon_{j j}\right)^{1 / 2}$.

In order to ensure that the water droplet remained roughly spherical in shape throughout the simulations, and also to prevent evaporation from the surface, all atoms contained in the system were subjected to a harmonic spherical boundary potential:

$$
V(\mathbf{R})= \begin{cases}\frac{1}{2} k_{\mathrm{B}}\left(|\mathbf{R}|-R_{\mathrm{B}}\right)^{2} & \text { if }|\mathbf{R}|-R_{\mathrm{B}}>0 \\ 0 & \text { otherwise }\end{cases}
$$

The radius $R_{\mathrm{B}}$ was set to $15.38 \AA$, so as to maintain the density of the droplet near $997 \mathrm{~kg} / \mathrm{m}^{3}$. The value of the force constant $k_{\mathrm{B}}$ was set to $0.5 \mathrm{kcal} \mathrm{mol}^{-1} \AA^{-2}$.

\section{S1.2 Initial Conditions}

Following Ruckenbauer and coworkers, ${ }^{6,7}$ when generating the initial conditions for the nonadiabatic molecular dynamics (MD) simulations, we adopted a hybrid procedure 
which treats the solute and solvent on a different footing. The positions and velocities of atoms comprising the solute molecule were selected in such a way as to include the zero-point energies of the intramolecular vibrational modes. The solvent was then thermalized in the presence of the solute. This approach is justified by the fact that the inclusion of zero-point energies in the intramolecular modes of the solute is required for a realistic calculation of spectral properties and relaxation dynamics. 8,9 However, the TIP3P/Fs water model was parameterized for use in purely classical MD simulations, and so the solvent must be treated entirely classically. Therefore, including zero-point energies in the solvent degrees of freedom is not desirable.

The ground-state equilibrium geometry of the isolated DMABN molecule was optimized at the SOS-MP2/cc-pVDZ level of theory. Subsequently, phase space points (i.e. sets of nuclear positions and velocities) were sampled from the Wigner distribution of the ground-state equilibrium geometry. As noted in Refs., ${ }^{10-12}$ semiclassical simulations of hydrogen-rich molecules are prone to an artificial leakage of zero-point energy from the stretching modes of hydrogen-heavy atom bonds to other vibrational modes. In order to mitigate this problem, when generating the Wigner distribution we froze the highest ten vibrational modes, which correspond to $\mathrm{C}-\mathrm{H}$ stretching modes.

Each of the molecular geometries sampled from the Wigner distribution was immersed in a water droplet consisting of 500 water molecules. Afterwards, the aqueous solvent was equilibrated with the solute by propagating a MD trajectory in the canonical $(N V T)$ ensemble. During the equilibration, the geometry of the DMABN molecule was kept frozen, and the potential energy surface of the entire system was calculated at the MM level of theory. The charge distribution of the solute was represented by a set of point charges placed at the nuclear positions. These charges were obtained by fitting them to the electrostatic potential generated by the relaxed SOS-MP2/cc-pVDZ electron density. The fit was performed with the use of the Merz-Singh-Kollman scheme ${ }^{13}$ as implemented in the computational chemistry software package Gaussian 16, Revision B.01. ${ }^{14}$ During the equilibration, the temperature of the solvent was maintained at $298 \mathrm{~K}$ by applying the Langevin thermostat with a friction coefficient of $\gamma=1 \mathrm{ps}$. The Langevin equation of motion was propagated with the use of the Brünger-Brooks-Karplus (BBK) integrator ${ }^{15}$ with a time step of $0.25 \mathrm{fs}$. The equilibration period lasted for $60 \mathrm{ps}$.

The equilibration step yielded a set of solute-solvent structures in which the solvent was in equilibrium with the solute. These structures were subsequently employed as the basis for the calculation of the photoabsorption spectrum using the semiclassical nuclear ensemble method. ${ }^{16,17}$ The resulting spectrum is shown in Figure S2 (b).

Afterwards, phase space points with excitation energies in the range of $4.6 \pm 0.1 \mathrm{eV}$, which covers the peak of the first photoabsorption band of DMABN in the water droplet, were randomly sampled from the simulated spectrum with probabilities proportional to their oscillator strengths. A set of 50 phase space points were selected in this manner, and each was used as the starting point for a single FSSH trajectory. The simulations were propagated for a time period of $1.2 \mathrm{ps}$.

The same general procedure was followed to generate the initial conditions for the 
Figure S2: Simulated photoabsorption spectra of DMABN in (a) the gas phase and (b) in the 500-molecule water droplet. The shaded area represents the energy interval from which initial conditions for the nonadiabatic molecular dynamics simulations were sampled.

(a) gas phase

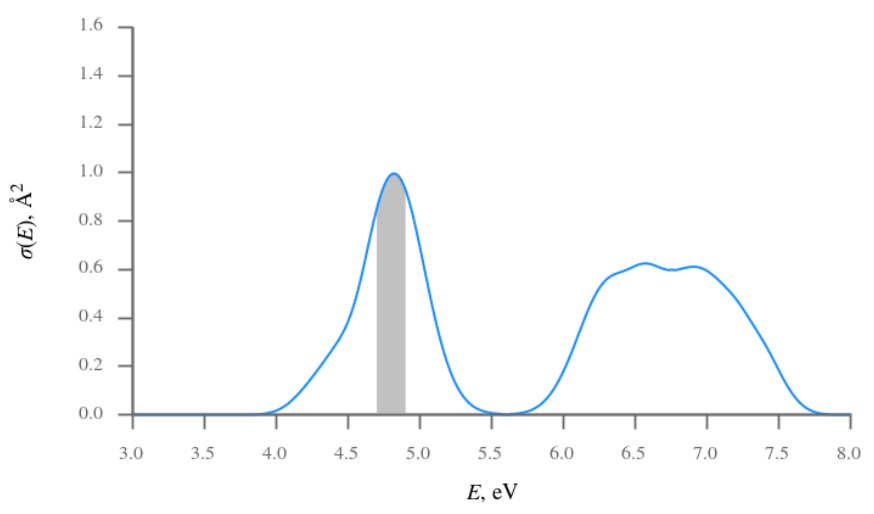

(b) water droplet

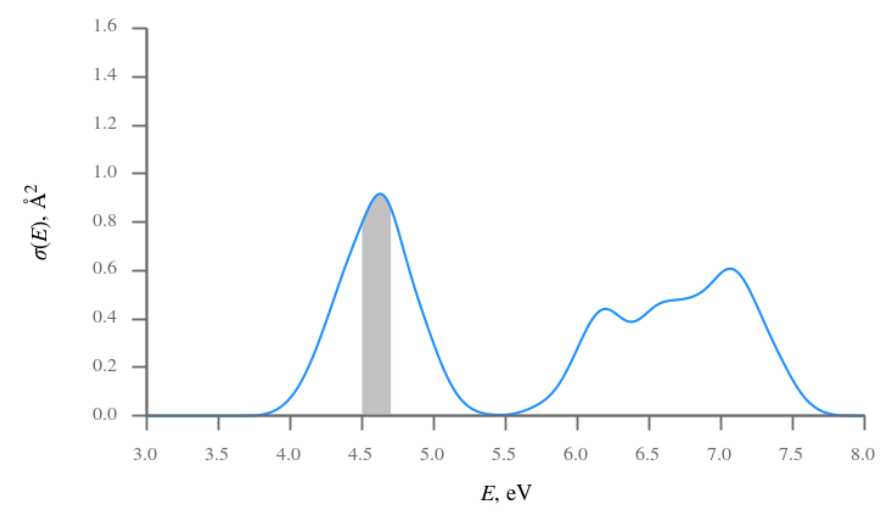

gas-phase simulations, except, of course, that the solvent and the equilibration stage were omitted. The simulated photoabsorption spectrum of DMABN in the gas phase is shown in Figure S2 (a). It can be seen that aqueous solvation causes a red shift of around $0.2 \mathrm{eV}$ in the position of the first photoabsorption band. Therefore, for the sake of consistency with the condensed-phase simulations, in the gas-phase simulations the photon energy interval from which the initial conditions were sampled was shifted upward by $0.2 \mathrm{eV}$. 


\section{S1.3 Nonadiabatic Molecular Dynamics}

The nonadiabatic MD simulations were carried out with the fewest switches surface hopping (FSSH) algorithm, adapted for use with the QM/MM method. The FSSH algorithm was implemented in a "wrapper" program which called Turbomole for the calculation of the electronic structure of the solute, then parsed the output, performed the MM part of the calculation, and propagated the equations of motion.

The FSSH simulations were carried out in the microcanonical ( $N V E$ ) ensemble. The dynamics of the nuclei was propagated according to Newton's equations of motion, and with the use of the velocity Verlet integrator with a timestep of $0.5 \mathrm{fs}$.

As is usual in the FSSH method, the electronic wavefunction of the QM subsystem was expanded in terms of a linear combination of adiabatic electronic states $\left\{\phi_{j}(\mathbf{r} ; \mathbf{R})\right\}$ with time-dependent complex coefficients $\left\{a_{j}(t)\right\}$ :

$$
\Phi(\mathbf{r}, t ; \mathbf{R})=\sum_{j} a_{j}(t) \phi_{j}(\mathbf{r} ; \mathbf{R})
$$

The $\mathrm{S}_{1}$ and $\mathrm{S}_{2}$ states were included in equation 6 . The time-evolution of the wavefunction expansion coefficients (equation 7 below) was integrated with the use of the Runge-Kutta fourth-order method with a time step of $0.0004 \mathrm{fs}$, using quantities interpolated linearly between successive classical steps.

$$
\mathrm{i} \hbar \dot{a}_{k}=\sum_{j} a_{j}\left(\delta_{j k} E_{k}(\mathbf{R})-\mathrm{i} \hbar C_{k j}\right)
$$

For the purpose of calculating the nonadiabatic coupling element (NACME) between the $\mathrm{S}_{1}$ and $\mathrm{S}_{2}$ states, the SOS-ADC(2) excited-state wavefunctions $\phi_{j}$ were approximated as wavefunctions of the configuration interaction singles (CIS) type:

$$
\left|\phi_{j}\right\rangle \approx \sum_{i, a} R_{i}^{a}(j)\left|\Phi_{i}^{a}\right\rangle \text { where }\left|\Phi_{i}^{a}\right\rangle=\hat{a}^{\dagger} \hat{i}\left|\Phi_{0}\right\rangle
$$

Here, $\left|\Phi_{0}\right\rangle$ is the RHF reference determinant, and $R_{i}^{a}(j)$ are elements of the Jacobian eigenvector of state $j$ corresponding to single excitations. The singles excitations part of the Jacobian eigenvector for each state was extracted from Turbomole output files with the use of the program 'bin2matrix' distributed as part of the simulation package Newton-X. ${ }^{18-21}$ Subsequently, the NACME was calculated using a finite differences formula: ${ }^{22}$

$$
C_{i j}(t+\Delta / 2)=\frac{1}{2 \Delta}\left[\left\langle\phi_{i}(t) \mid \phi_{j}(t+\Delta)\right\rangle-\left\langle\phi_{i}(t+\Delta) \mid \phi_{j}(t)\right\rangle\right]
$$

Here, $\Delta$ is the length of the classical time step (the time step used in the integration of Newton's equations of motion). The wavefunction overlap terms which appear in equation 9 were evaluated with the program of Plasser and coworkers. ${ }^{23,24}$

Furthermore, the time-evolution of the expansion coefficients $\left\{a_{j}(t)\right\}$ was corrected 
for decoherence via the scheme proposed by Granucci and Persico. ${ }^{25}$ The correction constant was set to $C=0.1 E_{\mathrm{h}}$.

The phases of electronic wavefunctions calculated by a quantum chemistry program such as Turbomole are set arbitrarily, and they can change from one timestep to another. In order to correct for this effect, the wavefunction phases were tracked by calculating overlap elements between electronic states at successive time steps: $\left\langle\phi_{j}(t+\Delta) \mid \phi_{j}(t)\right\rangle$. When a phase change was detected, the overlap elements involving the given electronic state were multiplied by -1 .

When the system underwent a nonadiabatic transition (or, 'hop') from one state to another, the velocities of the QM atoms (i.e., atoms comprising the inner subsystem) were rescaled in such a way as to conserve the total energy. The velocities of the MM atoms (outer subsystem) were left unchanged. This measure prevents an unphysical transfer of energy between the inner and outer subsystems during nonadiabatic transitions.

\section{S1.4 Choice of Electronic Structure Method}

Part of the challenge in simulating the relaxation process of DMABN lies in the sensitivity of its excited-state PESs to the choice of electronic structure method. ${ }^{26,27}$ Because the relevant excited-state structures of DMABN are close to one another in energy, even fairly small errors in relative energies may decisively alter the dynamics of the excited-state population. Thus, the simulation results will ultimately depend on the choice of methodology, which involves a certain degree of arbitrariness. For this reason, in the present section we explain the rationale behind our decision to use the SOS-ADC(2) method.

In many ways, the second-order algebraic-diagrammatic construction method $(\mathrm{ADC}(2))$ is the method of choice for large organic chromophores: it generally achieves reasonably good accuracy at a low computational cost, and is numerically stable even in the vicinity of state crossings. ${ }^{19}$ Furthermore, the availability of analytic gradients ${ }^{28}$ and approximate numerical nonadiabatic coupling elements ${ }^{19}$ paves the way for the application of this method in nonadiabatic MD simulations.

Nonetheless, the conventional variant of $\operatorname{ADC}(2)$ (which is to say, without a scaling of the same- and opposite-spin contributions to the correlation energy) is insufficiently accurate for the purposes of the present study. Although it correctly accounts for the existence of the $\mathrm{S}_{1}$-LE and the $\mathrm{S}_{1}$-TICT structures of DMABN, both of which appear as minima on the PES of the $\mathrm{S}_{1}$ state of the isolated molecule, ${ }^{26}$ it erroneously places the $\mathrm{S}_{1}$-TICT structure lower in energy than the $\mathrm{S}_{1}-\mathrm{LE}$ structure. In reality, the $\mathrm{S}_{1}$-TICT structure lies higher in energy than the $\mathrm{S}_{1}$-LE structure. ${ }^{29,30}$

The performance of the $\mathrm{ADC}(2)$ method for DMABN was recently analyzed by Mewes and coworkers, ${ }^{31}$ who demonstrated that $\mathrm{ADC}(2)$ also suffers from a more subtle deficiency. Namely, for the low-lying singlet excited states of DMABN, it consistently predicts larger electron-hole separations than do higher-level methods: equations of motion coupled cluster singles and doubles (EOM-CCSD), and the third-order algebraic-diagrammatic construction method $(\operatorname{ADC}(3)) .{ }^{31}$

At first glance, the artificial overstabilization of the $\mathrm{S}_{1}$-TICT structure precludes 
the use of $\mathrm{ADC}(2)$ for the purpose of modeling solvent effects in the relaxation process of DMABN. This is because it will unrealistically shift the dynamics of the excited-state population towards the $\mathrm{S}_{1}$-TICT structure under all circumstances. However, the performance of $\operatorname{ADC}(2)$ and related methods can be controlled to some extent through the application of the spin-component scaling (SCS) technique. ${ }^{32,33}$ SCS was originally introduced by Grimme ${ }^{32}$ for use with the MP2 method. In this approach, the same- and opposite-spin contributions to the MP2 correlation energy are rescaled, separately from one another, with the aim of achieving improved accuracy. On the basis of simple theoretical arguments, Grimme proposed the following values for the scaling parameters: $c_{\mathrm{OS}}=6 / 5$ for the opposite-spin contribution to the correlation energy, and $c_{\mathrm{SS}}=1 / 3$ for the opposite-spin contribution.

A special case of the general SCS technique is the spin-opposite scaling (SOS) variant proposed by Jung and coworkers. ${ }^{34}$ In the SOS variant, the same-spin contribution to the MP2 correlation energy is neglected completely $\left(c_{\mathrm{SS}}=0\right)$, whereas the opposite-spin contribution is retained with a scaling coefficient of $c_{\mathrm{OS}}=1.3$.

The SCS approach has been generalized to a range of single-reference excited-state methods. ${ }^{35-40}$ Notably, Hellweg and coworkers ${ }^{37}$ have introduced an SCS variant of the second-order approximate coupled-cluster (CC2) method, and proposed an extension of this model to the $\mathrm{ADC}(2)$ method, which is closely related to $\mathrm{CC} 2$. Subsequently, Winter and Hättig ${ }^{39}$ explicitly defined the SOS variant of the $\operatorname{ADC}(2)$ method. It should be noted here that Krauter and coworkers ${ }^{40}$ have independently derived another implementation of the $\operatorname{SOS}-\operatorname{ADC}(2)$ method, which is not identical to the one given in Refs. ${ }^{37,39}$ In the present study, when discussing the $\operatorname{SOS}-\operatorname{ADC}(2)$ method, we always refer to the original variant. ${ }^{37,39}$

In their analysis of the accuracy of the SCS-CC2 method, Hellweg et al. ${ }^{37}$ reported that the responsiveness of $0-0$ excitation energies to the imposition of SCS depends on the character of the excited state in question. On average, excitations into $\mathrm{n} \pi^{*}$-type states were substantially blue-shifted, whereas $\pi \pi^{*}$-type states were less sensitive to SCS. While the physical reasons for the different sensitivity of $\mathrm{n} \pi^{*}$ - and $\pi \pi^{*}$-type states have not been explained to date, this observation raises the hope that the SCS procedure could be employed to mitigate the overstabilization of the $\mathrm{S}_{1}$-TICT structure of DMABN by $\mathrm{ADC}(2)$. This is because, in the $\mathrm{S}_{1}$-TICT structure, the $\mathrm{p}_{z}$ atomic orbital of the dimethylamino group nitrogen lies roughly in the plane of the phenyl ring (see Figure S3 below). Consequently, the $\mathrm{S}_{1}\left({ }^{1} \mathrm{~L}_{\mathrm{a}}\right)$ state, which features charge transfer from the dimethylamino group nitrogen onto the phenyl ring, shows some resemblance to an $\mathrm{n} \pi^{*}$-type state.

Accordingly, we undertook to verify whether the description of the excited-state PESs of DMABN by the ADC(2) method is improved by the imposition of SCS. To this end, we optimized the equilibrium geometries on the $\mathrm{S}_{1}$ PES, firstly with the use of the conventional $\mathrm{ADC}(2)$ method, and secondly with its SOS variant. The reason that we opted to use the SOS variant is because it represents the limiting case of the general SCS approach (which is to say, only the opposite-spin contributions to the correlation energy are retained; the same-spin contributions are neglected). 
The other simulation settings were the same as specified in the Section 2.2 of the main body of this paper.

The effects of imposing the SOS procedure are illustrated in Figure S4, which compares the energy level diagrams for DMABN obtained with the conventional MP2 and $\mathrm{ADC}(2)$ methods, and with their SOS variants. (NB for technical reasons, zero-point vibrational energy corrections are not included in these diagrams.) Whereas the conventional $\operatorname{ADC}(2)$ calculation erroneously places the $\mathrm{S}_{1}$-TICT structure lower in energy than the $\mathrm{S}_{1}-\mathrm{LE}$ structure by $0.249 \mathrm{eV}$, the imposition of the SOS procedure inverts the energy ordering of the two excited-state structures, such that $\mathrm{S}_{1}$-TICT becomes higher in energy than $\mathrm{S}_{1}-\mathrm{LE}$ by $0.119 \mathrm{eV}$. In other respects, the results of the conventional and SOS calculations are in close agreement with one another.

Taking into account zero-point vibrational energy (ZPVE) corrections, the energy difference between the $\mathrm{S}_{1}$-TICT and $\mathrm{S}_{1}$-LE structures at the SOS-ADC(2)/cc-pVDZ level of theory is $0.148 \mathrm{eV}$. This coincides closely with the best available theoretical benchmark, which is the value of $0.17 \mathrm{eV}$, obtained by Köhn and Hättig ${ }^{29}$ at the CC2/TZVPP level of theory (including a correction for higher-order electron correlation effects from the $\left.\operatorname{CCSDR}(3) \operatorname{method}^{41}\right)$. The close agreement of the SOS-ADC(2) result with this reference value may be fortuitous to some extent, since the SOS-ADC $(2)$ calculation was performed with the relatively small cc-pVDZ basis set, and is not converged with respect to basis set size. Nevertheless, it is evident that the imposition of the SOS precedure at least partially corrects for the overstabilization of the $\mathrm{S}_{1}$-TICT structure by conventional $\operatorname{ADC}(2)$. This makes $\operatorname{SOS}-\operatorname{ADC}(2)$ a good choice of electronic structure method for the simulation of the relaxation process of DMABN in polar solution.

Figure S3: A schematic illustration of the orientation of the $\mathrm{p}_{z}$ atomic orbital of the dimethylamino group nitrogen in the $\mathrm{S}_{1}$-TICT structure of DMABN.

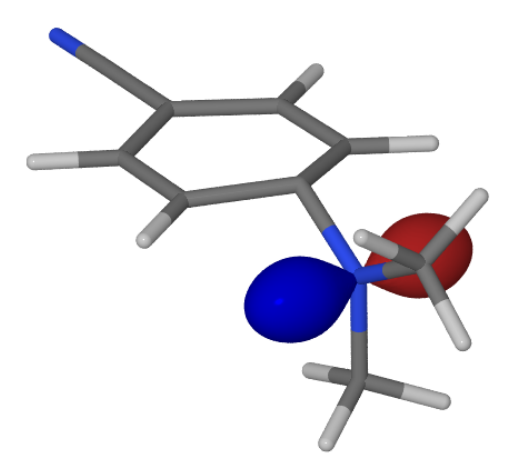


Figure S4: Energy level diagrams for DMABN, obtained with the use of the conventional MP2 and $\operatorname{ADC}(2)$ methods (left), and with the imposition of the SOS procedure (right). The energy levels represent adiabatic energies calculated at the ground- and excited-state equilibrium geometries. The electronic state on which a given structure was optimized is marked with a bullseye symbol. The diabatic character of each state is indicated with the use of color.

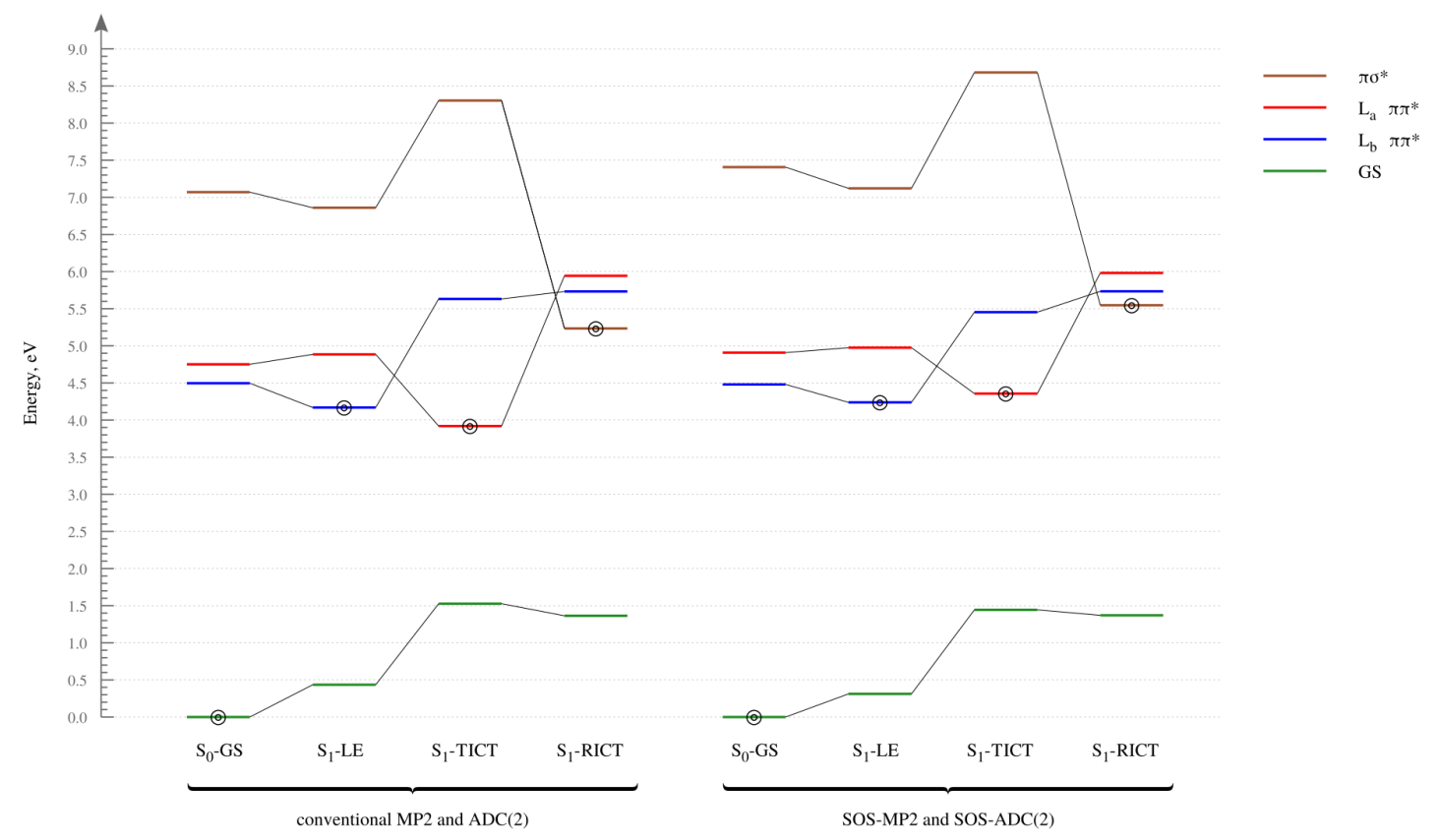

\section{S1.5 Spin-Opposite Scaling and Electronic Structure}

In the previous section, we showed that the SOS procedure improves the accuracy of $\mathrm{ADC}(2)$ for the relative energies of the $\mathrm{S}_{1}-\mathrm{LE}$ and $\mathrm{S}_{1}$-TICT structures of DMABN. It is also of interest to examine how the application of SOS affects the electronic structures of the various excited states, and that will be our aim in this section. For this purpose, we will employ the cosine similarity diagnostic introduced in Ref. ${ }^{42}$ This diagnostic works by comparing the electronic structures of excited states obtained with two different methods in terms of the dominant electronic configurations. Here, we make the approximation that each $\operatorname{ADC}(2)$ excited state $\left|\phi_{m}\right\rangle$, and also each $\operatorname{SOS}-\operatorname{ADC}(2)$ excited state $\left|\phi_{n}\right\rangle$, 
are represented by a wavefunction of the CIS type:

$$
\begin{aligned}
& \left|\phi_{m}\right\rangle \approx \sum_{i, a} R_{i}^{a}(m)\left|\Phi_{i}^{a}\right\rangle \\
& \left|\phi_{n}\right\rangle \approx \sum_{i, a} R_{i}^{a}(n)\left|\Phi_{i}^{a}\right\rangle
\end{aligned}
$$

As per the usual convention, index $i$ runs over occupied orbitals, while index $a$ runs over virtual orbitals. $R_{i}^{a}(m)$ are elements of the singles part of the Jacobian eigenvector for the $m$-th $\mathrm{ADC}(2)$ state. Likewise, $R_{i}^{a}(n)$ are elements of the singles part of the Jacobian eigenvector for the $n$-th $\operatorname{SOS}-\operatorname{ADC}(2)$ state. Lastly, $\left|\Phi_{i}^{a}\right\rangle=\hat{a}^{\dagger} \hat{i}\left|\Phi_{0}\right\rangle$ is the singly excited determinant generated from the reference determinant by exciting an electron from the $i$-th occupied orbital to the $a$-th virtual orbital. This approximation is entirely analogous to the one made when calculating the NACMEs between excited states; see Section S1.3 in this document.

The degree of resemblance $(S(m, n))$ between the $m$-th $\operatorname{ADC}(2)$ state and the $n$-th SOS-ADC $(2)$ state is then calculated as the absolute value of the cosine similarity between the singles parts of the respective Jacobian eigenvectors:

$$
S(m, n)=\frac{\left|\sum_{i, a} R_{i}^{a}(m) R_{i}^{a}(n)\right|}{\sqrt{\sum_{i, a}\left[R_{i}^{a}(m)\right]^{2}} \sqrt{\sum_{i, a}\left[R_{i}^{a}(n)\right]^{2}}}
$$

The diagnostic $S(m, n)$ takes values ranging from 0 , which indicates no resemblance between the Jacobian eigenvectors of a given pair of $\operatorname{ADC}(2)$ and $\operatorname{SOS}-\operatorname{ADC}(2)$ states, to 1 , which means that the two states are characterized by identical Jacobian eigenvectors. Intermediate values indicate partial resemblance between the Jacobian eigenvectors. Thus, $S(m, n)$ provides a quantitative state-by-state comparison between the excited electronic states obtained with the conventional $\operatorname{ADC}(2)$ and the SOS-ADC(2) methods. It is convenient to think of the values of $S(m, n)$ as forming a matrix, in which $m$, the index of the $\operatorname{ADC}(2)$ state, identifies the row, and $n$, the index of the SOS-ADC(2) state, identifies the column.

Throughout the analysis, we employed the aug-cc-pVDZ basis set, ${ }^{43}$ which expands on the cc-pVDZ basis set through the addition of diffuse basis functions on all atoms. It is expected that increasing the flexibility of the basis set will accentuate any differences between the conventional and SOS calculations, thereby making the test more stringent. Moreover, the addition of diffuse basis functions allows us to detect the Rydberg-type excited states of DMABN. The Rydberg-type states are not known to be involved in dual fluorescence mechanism, but from a methodological standpoint, it is nevertheless informative to include them in the analysis.

The ground-state equilibrium geometry of DMABN was optimized at the SOS-MP2 level of theory. Subsequently, the vertical excitation spectrum was calculated with the use of the conventional $\operatorname{ADC}(2)$ method, and with the SOS-ADC(2) method. In either 
case, singlet excited states ranging from $\mathrm{S}_{1}$ to $\mathrm{S}_{8}$ were included in the calculation. The resulting vertical excitation spectra are summarized in Table S2. Accompanying this information, Figure S5 provides a visual representation of the values of the cosine similarity diagnostic $S(m, n)$. Specifically, the value of $S(m, n)$ for a given pair of $\operatorname{ADC}(2)$ and SOS-ADC(2) states is indicated with the use of color.

In the upper left $6 \times 6$ sub-block of the $S(m, n)$ matrix, the diagonal elements take values close to unity, while most of the off-diagonal elements are close to zero. This shpws that for singlet excited states from $\mathrm{S}_{1}$ to $\mathrm{S}_{6}$, there is a one-on-one correspondence between the excited electronic states obtained with the $\operatorname{ADC}(2)$ and the SOS-ADC(2) methods, and, furthermore, that both methods predict the same energy ordering of the excited states. In other words, the $\mathrm{S}_{1}$ state obtained with $\operatorname{ADC}(2)$ is closely similar to the $\mathrm{S}_{1}$ state obtained with $\operatorname{SOS}-\operatorname{ADC}(2)$, and so on, up until the $\mathrm{S}_{6}$ state.

Interestingly, the diagnostic $S(m, n)$ takes moderately large values for the similarity between the $\mathrm{S}_{2} \mathrm{ADC}(2)$ state and the $\mathrm{S}_{3} \operatorname{SOS}-\operatorname{ADC}(2)$ state, and likewise for similarity between the $\mathrm{S}_{3} \operatorname{ADC}(2)$ state and the $\mathrm{S}_{2} \operatorname{SOS}-\operatorname{ADC}(2)$ state. The reason for this is simple. At the conventional $\operatorname{ADC}(2)$ level, the $\mathrm{S}_{2}$ state is nominally of ${ }^{1} \mathrm{~L}_{\mathrm{a}}$ diabatic character, but it mixes with the $\mathrm{S}_{3}$ state, which is a Rydberg state. Because the $\mathrm{S}_{3}$ state has an admixture of ${ }^{1} \mathrm{~L}_{\mathrm{a}}$ character, the $\mathrm{S}_{0} \rightarrow \mathrm{S}_{3}$ transition shows an appreciably large oscillator strength (see Table S2). The application of the SOS procedure apparently reduces mixing between ${ }^{1} \mathrm{~L}_{\mathrm{a}}$ and Rydberg character in the $\mathrm{S}_{2}$ and $\mathrm{S}_{3}$ states. This interpretation is supported by the fact that the SOS procedure increases the oscillator strength for the $\mathrm{S}_{0} \rightarrow \mathrm{S}_{2}$ transition, and decreases the oscillator strength for the $\mathrm{S}_{0} \rightarrow \mathrm{S}_{3}$ transition. In the $S(m, n)$ matrix, this manifests itself in the form of relatively large off-diagonal values for similarity between the $\mathrm{S}_{2}$ and $\mathrm{S}_{3}$ state.

As regards the $S_{7}$ and $S_{8}$ states, we find that the imposition of the SOS procedure causes a reordering of these two states. That is to say, the $\mathrm{S}_{7} \operatorname{ADC}(2)$ state is a counterpart of the $\mathrm{S}_{8} \mathrm{SOS}-\mathrm{ADC}(2)$ state, whereas the $\mathrm{S}_{8} \operatorname{ADC}(2)$ state shows close similarity to the $\mathrm{S}_{7} \mathrm{SOS}-\mathrm{ADC}(2)$ state. This is clearly reflected by the $S(m, n)$ matrix: in the bottom right $2 \times 2$ sub-block of that matrix, the diagonal elements are near-zero, whereas the off-diagonal elements are close to unity. This shows that the energy ordering of the two states is inverted, but the electronic structure of each state is largely unchanged by the application of the SOS procedure.

In summary, the effects of imposing the SOS procedure on the electronic structures of the relevant singlet excited states of DMABN are rather slight. For states up to and including $\mathrm{S}_{8}$, there is a one-on-one correspondence between the states obtained with the conventional $\operatorname{ADC}(2)$ method, and those obtained with the SOS-ADC(2) method. This finding should be regarded as encouraging, since any major discrepancies between the electronic structures obtained with these two methods would have cast doubt on the reliability of both of them. 
Table S2: Vertical excitation spectrum of DMABN, calculated with the use of the conventional $\operatorname{ADC}(2)$ method, and with the imposition of the SOS procedure: vertical excitation energies $(\Delta E)$ and associated oscillator strengths $(f)$. Both calculations were performed at the ground-state equilibrium geometry as optimized at the SOS-MP2/aug-cc-pVDZ level. The Rydberg-type states are labeled as ${ }^{1} \pi \mathrm{R}$ states.

\begin{tabular}{llll}
\hline Level of theory & State & $\Delta E, \mathrm{eV}$ & $f$ \\
\hline ADC(2)/aug-cc-pVDZ & $\mathrm{S}_{1}\left({ }^{1} \mathrm{~L}_{\mathrm{b}}\right)$ & 4.303 & 0.0264 \\
& $\mathrm{~S}_{2}\left({ }^{1} \mathrm{~L}_{\mathrm{a}}\right)$ & 4.496 & 0.4058 \\
& $\mathrm{~S}_{3}\left({ }^{1} \pi \mathrm{R}\right)$ & 4.825 & 0.1323 \\
& $\mathrm{~S}_{4}\left({ }^{1} \pi \mathrm{R}\right)$ & 5.286 & 0.0006 \\
& $\mathrm{~S}_{5}\left({ }^{1} \pi \mathrm{R}\right)$ & 5.408 & 0.0018 \\
& $\mathrm{~S}_{6}\left({ }^{1} \pi \mathrm{R}\right)$ & 5.572 & 0.0259 \\
& $\mathrm{~S}_{7}\left({ }^{1} \pi \mathrm{R}\right)$ & 5.816 & 0.0058 \\
& $\mathrm{~S}_{8}\left({ }^{1} \pi \pi^{*}\right)$ & 5.914 & 0.1388 \\
\hline SOS-ADC(2)/aug-cc-pVDZ & $\mathrm{S}_{1}\left({ }^{1} \mathrm{~L}_{\mathrm{b}}\right)$ & 4.345 & 0.0187 \\
& $\mathrm{~S}_{2}\left({ }^{1} \mathrm{~L}_{\mathrm{a}}\right)$ & 4.710 & 0.4889 \\
& $\mathrm{~S}_{3}\left({ }^{1} \pi \mathrm{R}\right)$ & 5.185 & 0.0608 \\
& $\mathrm{~S}_{4}\left({ }^{1} \pi \mathrm{R}\right)$ & 5.688 & 0.0001 \\
& $\mathrm{~S}_{5}\left({ }^{1} \pi \mathrm{R}\right)$ & 5.803 & 0.0010 \\
& $\mathrm{~S}_{6}\left({ }^{1} \pi \mathrm{R}\right)$ & 5.925 & 0.0367 \\
& $\mathrm{~S}_{7}\left({ }^{1} \pi \pi^{*}\right)$ & 6.134 & 0.2241 \\
& $\mathrm{~S}_{8}\left({ }^{1} \pi \mathrm{R}\right)$ & 6.181 & 0.0017 \\
\hline
\end{tabular}


Figure S5: Visual representation of cosine similarity between single excitations vectors of low-lying singlet excited states of DMABN calculated at the $\mathrm{ADC}(2) /$ aug-cc-pVDZ and SOS-ADC(2)/aug-cc-pVDZ levels of theory. Values of the $S(m, n)$ diagnostic are plotted in matrix form; color represents the value of $S(m, n)$ for a given pair of excited states obtained with $\operatorname{ADC}(2)$ and $\operatorname{SOS}-\operatorname{ADC}(2)$.

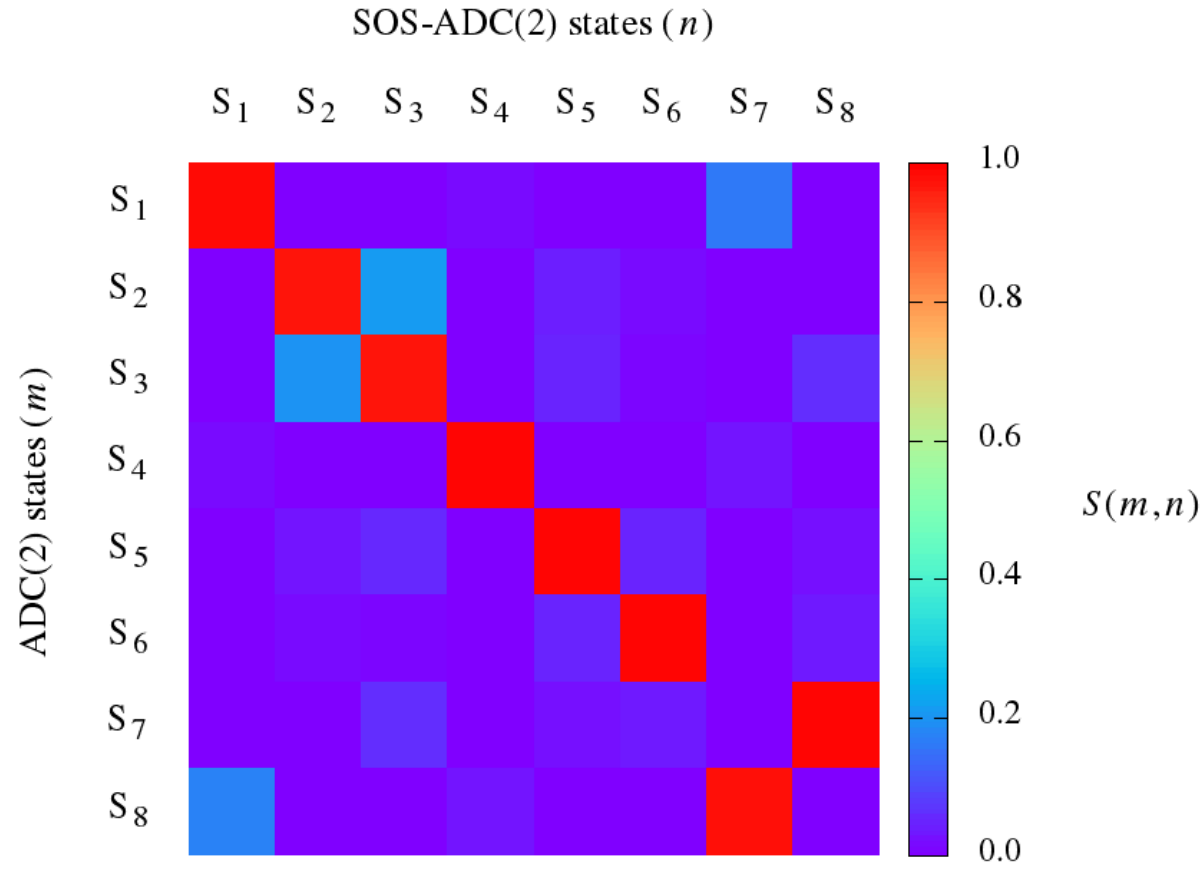




\section{S1.6 ADC(3) Calculation of Relative Energies}

A reviewer noted that the accuracy of the SOS-ADC(2) method for the excited-state PESs of DMABN should be assessed against a benchmark provided by a more accurate electronic structure method. The highest-level method that we are able to apply to the relatively large DMABN molecule is the $\operatorname{ADC}(3)$ method, ${ }^{44,45}$ and accordingly we chose to employ that method as a benchmark for SOS-ADC(2).

For the purposes of the benchmarking, the ground-state equilibrium geometry $\left(\mathrm{S}_{0}-\mathrm{GS}\right)$ was reoptimized at the SOS-MP2/cc-pVTZ level of theory, and the excited-state structures were reoptimized at the SOS-ADC(2)/cc-pVTZ level.

Afterwards, the energies of the resulting equilibrium geometries were recalculated wtih the $\operatorname{ADC}(3)$ method. These single-point $\operatorname{ADC}(3)$ calculations were performed within the program ADC-connect. ${ }^{46-48}$ The 6-311G(d) basis set ${ }^{49}$ was employed. Spin-component scaling was not imposed. The reference RHF calculations were done with the program PySCF. ${ }^{50}$ ZPVE corrections to the single-point ADC(3)/6-311G(d) energies were taken from the $\operatorname{SOS}-\operatorname{ADC}(2) / c c-p V T Z$ calculations. In what follows, we will denote this level of theory with the shorthand $\mathrm{ADC}(3) / / \operatorname{SOS}-\mathrm{ADC}(2)$.

The energies of the excited-state structures of DMABN obtained at the SOS-ADC(2)/cc-pVDZ and ADC(3)//SOS-ADC(2) levels are listed in Table S3 below. For easier reference, the same data is shown in Figure S6 on the following page. It can be seen that both methods predict the same energy ordering of the three excited-state structures. In quantitative terms, however, there are non-negligible differences between the relative energies calculated with the two methods. In particular, at the SOS-ADC(2)/cc-pVTZ level of theory, the energy difference between the $\mathrm{S}_{1}$-LE and $\mathrm{S}_{1}$-TICT structures is $0.216 \mathrm{eV}$, but on going to the $\mathrm{ADC}(3) / / \mathrm{SOS}-\mathrm{ADC}(2)$ level, it increases to $0.307 \mathrm{eV}$. Furthermore, the $\mathrm{ADC}(3) / / \mathrm{SOS}-\mathrm{ADC}(2)$ calculation places the $\mathrm{S}_{1}$-RICT structure $0.383 \mathrm{eV}$ lower in energy (relative to the $\mathrm{S}_{0}$-GS structure) than does SOS-ADC (2)/cc-pVTZ. Still, the differences between the energies obtained at the SOS-ADC $(2) / /$ cc-pVTZ and at the ADC(3)//SOS-ADC(2) levels seem small enough that $\mathrm{SOS}-\mathrm{ADC}(2)$ can be considered as accurate enough for use in nonadiabatic molecular dynamics simulations.

Table S3: Energies of the excited-state structures of DMABN as calculated at the SOS-ADC(2)/cc-pVTZ and ADC(3)//SOS-ADC(2) levels of theory. The energy values are given relative to the energy of the $\mathrm{S}_{0}-\mathrm{GS}$ structure at the given level of theory, and include ZPVE corrections calculated at the SOS-ADC(2)/cc-pVTZ level.

\begin{tabular}{lll}
\hline Structure & \multicolumn{2}{c}{$E, \mathrm{eV}$} \\
& SOS-ADC(2)/cc-pVTZ & ADC(3)//SOS-ADC(2) \\
\hline $\mathrm{S}_{1}$-LE & 4.080 & 4.116 \\
$\mathrm{~S}_{1}$-TICT & 4.296 & 4.423 \\
$\mathrm{~S}_{1}$-RICT & 5.414 & 5.031 \\
\hline
\end{tabular}


Figure S6: Energy level diagram for DMABN obtained at the SOS-ADC(2)/cc-pVTZ and $\operatorname{ADC}(3) / / \operatorname{SOS}-\mathrm{ADC}(2)$ levels of theory. The origin of the energy scale is set to the energy of the $\mathrm{S}_{0}$-GS structure. The energies include ZPVE corrections calculated at the SOS-ADC(2)/cc-pVTZ level.

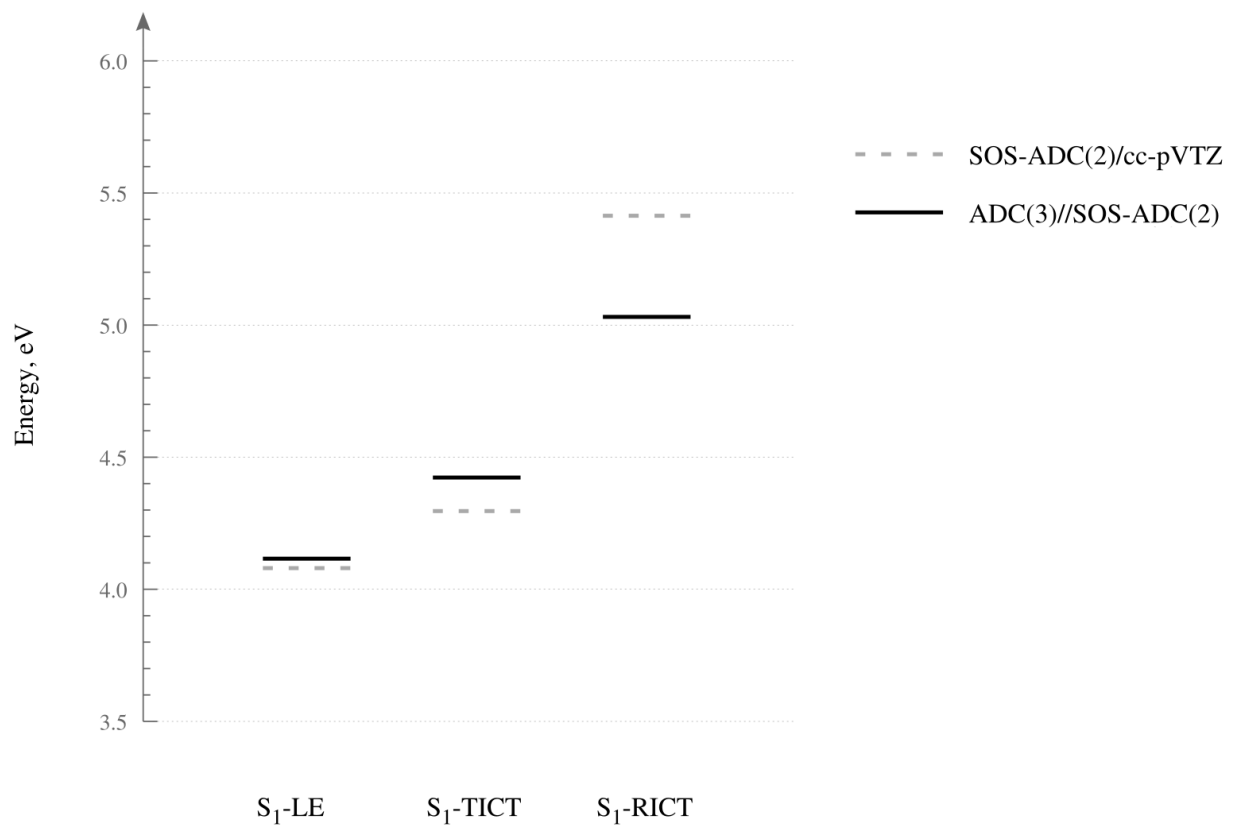




\section{S2 Electronic Excitation Spectrum of DMABN}

In this section, we examine the structures of the relevant excited electronic states of DMABN, using the same level of theory (SOS-ADC(2)/cc-pVDZ) as was employed in the nonadiabatic molecular dynamics simulations. The character of each excited state was characterized by calculating natural transition orbitals ${ }^{51}$ (NTOs) for the transition from the singlet ground state to the given excited state.

The calculated vertical excitation spectrum is characterized in Table S4. In line with previous $\mathrm{CC} 2^{29}$ and complete active space second-order perturbation theory ${ }^{30}$ (CASPT2) calculations, we find that the lowest vertical excitation is into the ${ }^{1} \mathrm{~L}_{\mathrm{b}}$ state. The dominant NTOs for the transition from the $\mathrm{S}_{0}$ state into the $\mathrm{S}_{1}\left({ }^{1} \mathrm{~L}_{\mathrm{b}}\right)$ state are plotted in Figure $\mathrm{S} 7$ (a). It can be seen that the $\mathrm{S}_{1}\left({ }^{1} \mathrm{~L}_{\mathrm{b}}\right)$ state is described by two NTO pairs with appreciably large eigenvalues, which indicates that it exhibits substantial multiconfigurational character.

Table S4: Vertical excitation spectrum of DMABN calculated at the SOS-ADC (2)/cc-pVDZ level of theory: vertical excitation energies $(\Delta E)$ and associated oscillator strengths $(f) . \mu$ is the (orbital-relaxed) electric dipole moment of the given state.

\begin{tabular}{llll}
\hline State & $\Delta E, \mathrm{eV}$ & $f$ & $\mu, \mathrm{D}$ \\
\hline $\mathrm{S}_{0}$ & & & 6.62 \\
$\mathrm{~S}_{1}\left({ }^{1} \mathrm{~L}_{\mathrm{b}}\right)$ & 4.479 & 0.0178 & 7.73 \\
$\mathrm{~S}_{2}\left({ }^{1} \mathrm{~L}_{\mathrm{a}}\right)$ & 4.909 & 0.5557 & 11.80 \\
$\mathrm{~S}_{7}\left({ }^{1} \pi \sigma^{*}\right)$ & 7.407 & $8 \times 10^{-6}$ & 12.71 \\
\hline
\end{tabular}

Although the ${ }^{1} \mathrm{~L}_{\mathrm{b}}$ state is conventionally called the locally excited (LE) state, careful inspection of the dominant NTOs reveals that this label is somewhat misleading - in reality, the electronic excitation is delocalized over the entire out-of-plane $\pi$-bonding system.

The ${ }^{1} \mathrm{~L}_{\mathrm{b}}$ state is closely followed by the ${ }^{1} \mathrm{~L}_{\mathrm{a}}$ state, which is again in line with the results of previous calculations. ${ }^{29,30}$ As shown in Figure S7 (b), the transition from the ground state into the $\mathrm{S}_{2}\left({ }^{1} \mathrm{~L}_{\mathrm{b}}\right)$ state is dominated by a single NTO pair, and involves charge transfer from the dimethylamino group nitrogen onto the benzonitrile moiety.

The ${ }^{1} \pi \sigma^{*}$ state is well separated in energy from the ${ }^{1} \mathrm{~L}_{\mathrm{b}}$ and ${ }^{1} \mathrm{~L}_{\mathrm{a}}$ states, and appears as the $\mathrm{S}_{7}$ adiabatic state. It arises mainly from the excitation of an electron from an out-of-plane $\pi$-type orbital into the out-of-plane $\pi^{*}$-type orbital of the nitrile group. 
Figure S7: Dominant NTOs for transitions from the the singlet ground state into the $\mathrm{S}_{1}\left({ }^{1} \mathrm{~L}_{\mathrm{b}}\right), \mathrm{S}_{2}\left({ }^{1} \mathrm{~L}_{\mathrm{a}}\right)$ and $\mathrm{S}_{7}\left({ }^{1} \pi \sigma^{*}\right)$ states of DMABN, plotted in the form of isosurfaces with isovalues of $\pm 0.05 a_{0}{ }^{-3 / 2}$. The eigenvalue $\left(\lambda_{i}\right)$ for each hole-particle NTO pair is given in terms of a percentage contribution. The calculation was performed at the SOS-ADC(2)/cc-pVDZ level of theory.
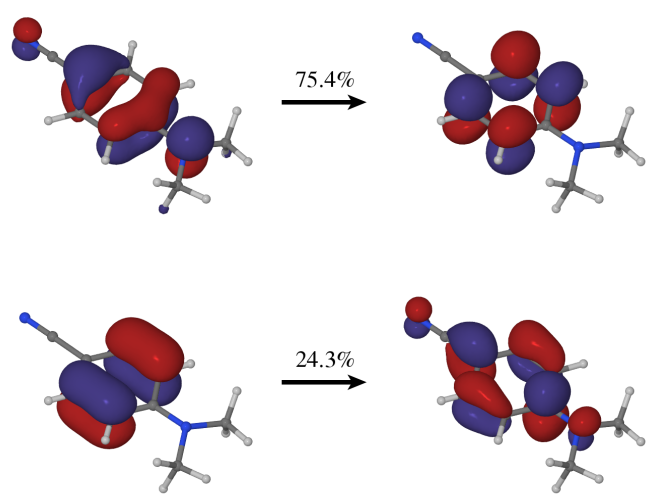

(a) $\mathrm{S}_{0} \rightarrow \mathrm{S}_{1}\left({ }^{1} \mathrm{~L}_{\mathrm{b}}\right)$
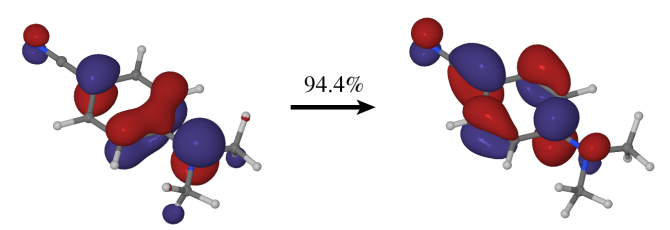

(b) $\mathrm{S}_{0} \rightarrow \mathrm{S}_{2}\left({ }^{1} \mathrm{~L}_{\mathrm{a}}\right)$

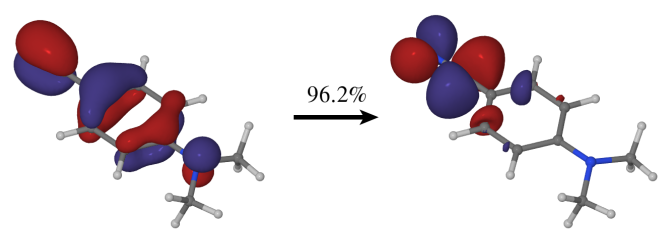

(c) $\mathrm{S}_{0} \rightarrow \mathrm{S}_{7}\left({ }^{1} \pi \sigma^{*}\right)$ 


\section{S3 Structure of the $\mathrm{S}_{2} / \mathrm{S}_{1}$ Conical Intersection of DMABN}

A reviewer asked the insightful question of whether there exists a conical intersection (CI) between the $\mathrm{S}_{2}$ and $\mathrm{S}_{1}$ states of DMABN as calculated with the SOS-ADC(2) method. In order to answer that, we have confirmed that a $\mathrm{S}_{2} / \mathrm{S}_{1}$ CI seam does indeed exist and, moreover, we have optimized the minimum-energy geometry (MCI) along this CI seam.

The MCI optimization was performed with the penalty function method of Ciminelli and coworkers. ${ }^{52}$ In this approach, the quantity being optimized is the penalty function defined as:

$$
f(\mathbf{R})=\frac{E_{2}(\mathbf{R})+E_{1}(\mathbf{R})}{2}+c_{1} c_{2}{ }^{2} \ln \left[1+\left(\frac{E_{2}(\mathbf{R})-E_{1}(\mathbf{R})}{c_{2}}\right)^{2}\right]
$$

where $\mathbf{R}$ denotes the molecular geometry, and $E_{2}(\mathbf{R})$ and $E_{1}(\mathbf{R})$ are the energies of the $S_{2}$ and $S_{1}$ states. The minimum of the penalty function coincides closely - but not exactly - with the true location of the MCI. The parameters $c_{1}$ and $c_{2}$ were set to the values recommended by Ciminelli et al: ${ }^{52} c_{1}=5(\mathrm{kcal} / \mathrm{mol})^{-1}$ and $c_{2}=5 \mathrm{kcal} / \mathrm{mol}$. The excited-state PESs were calculated at the SOS-ADC(2)/cc-pVDZ level of theory. The penalty function was minimized with the use of the limited-memory variant of the Broyden-Fletcher-Goldfarb-Shanno (BFGS) optimization algorithm.

The resulting MCI geometry is shown in Figure S8 (a). At this geometry, the energy gap between the $\mathrm{S}_{2}$ and $\mathrm{S}_{1}$ states is only $0.0005 \mathrm{eV}$, which is small enough that these states can be said to be degenerate to within the accuracy of the geometry optimization scheme. The six-membered ring is near-planar, while dimethylamino group is partially twisted with respect to the plane of the ring.

It is also of some interest to examine the topography of the PESs of the intersecting states in the branching space, which is spanned by the degeneracy-lifting coordinates. These are the gradient difference vector $\mathbf{g}_{21}$ and the nonadiabatic coupling vector $\left(\mathrm{NACV}, \mathbf{d}_{21}\right)$, given by:

$$
\mathbf{g}_{21}=\frac{\partial E_{2}(\mathbf{R})}{\partial \mathbf{R}}-\frac{\partial E_{1}(\mathbf{R})}{\partial \mathbf{R}}
$$

and

$$
\mathbf{d}_{21}=\left\langle\phi_{2}\left|\nabla_{\mathbf{R}}\right| \phi_{1}\right\rangle
$$

Because the analytic calculation of the NACV is not available at the $\mathrm{ADC}(2)$ level of theory (with or without the imposition of the SOS procedure), the NACV was instead calculated with the use of the extended multi-state complete active space second-order perturbation theory ${ }^{53}$ (XMS-CASPT2) method.

The XMS-CASPT2 calculation was performed with the program BAGEL, ${ }^{54,55}$ version 1.1.2. The active space in the underlying complete active space self-consistent field $^{56}$ (CASSCF) calculation consisted of 12 electrons distributed in the 11 
Figure S8: Characterization of the $\mathrm{S}_{2} / \mathrm{S}_{1} \mathrm{MCI}$ of DMABN. The MCI was optimized at the SOS-ADC $(2) / c c-p V D Z$ level of theory, and the same method was used in the calculation of the gradient difference vector. The NACV between the intersecting states was calculated at the XMS-CASPT2/cc-pVDZ level.

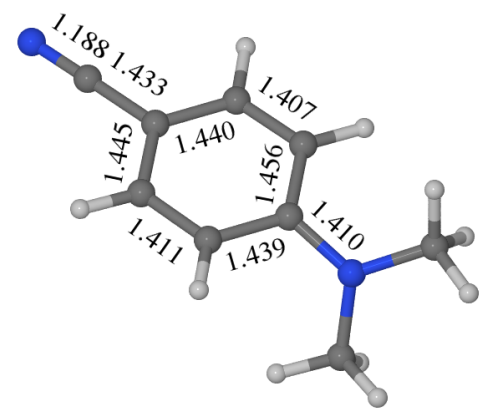

(a) molecular geometry

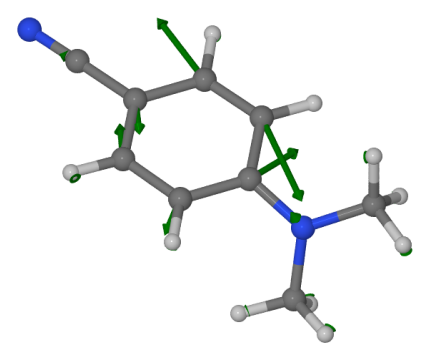

(b) gradient difference $\left(\mathbf{g}_{21}\right)$

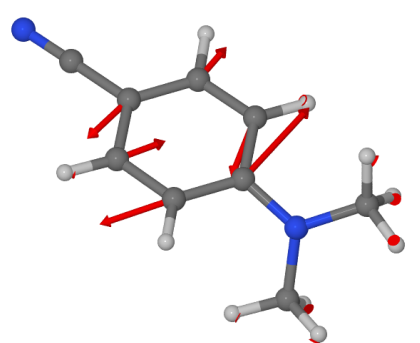

(c) $\operatorname{NACV}\left(\mathbf{d}_{21}\right)$

$\pi$ - and $\pi^{*}$-type orbitals which arise mainly from the $2 \mathrm{p}$ atomic orbitals of the carbon and nitrogen atoms. A state-averaging scheme was imposed in which the CASSCF orbitals were averaged over the lowest three singlet states. At the XMS-CASPT2 stage, the single state-single reference (SS-SR) contraction scheme ${ }^{57}$ was used, and the vertical shift was set to $0.2 E_{\mathrm{h}}$. The cc-pVDZ basis set was used in combination with the default density fitting basis set from the BAGEL library.

The gradient difference vector and the NACV are shown in Figure S8 (b) and (c), respectively. Both the branching space vectors correspond to different in-plane deformations of the six-membered ring, which is in line with the findings of the earlier studies by Gómez and coworkers ${ }^{58}$ and Georgieva et al. ${ }^{59}$

As a sidenote, at the MCI geometry optimized at the SOS-ADC(2)/cc-pVDZ level of theory, the XMS-CASPT2/cc-pVDZ calculation predicts a fairly large energy gap of 
Figure S9: Topography of the $\mathrm{S}_{2}$ and $\mathrm{S}_{1}$ states in the branching space the $\mathrm{S}_{2} / \mathrm{S}_{1}$ MCI, as calculated at the SOS-ADC(2)/cc-pVDZ level of theory. The MCI itself is located at the 0,0 point on the horizontal plane. The zero of the energy scale corresponds to the energy of the $\mathrm{S}_{0}$-GS structure.

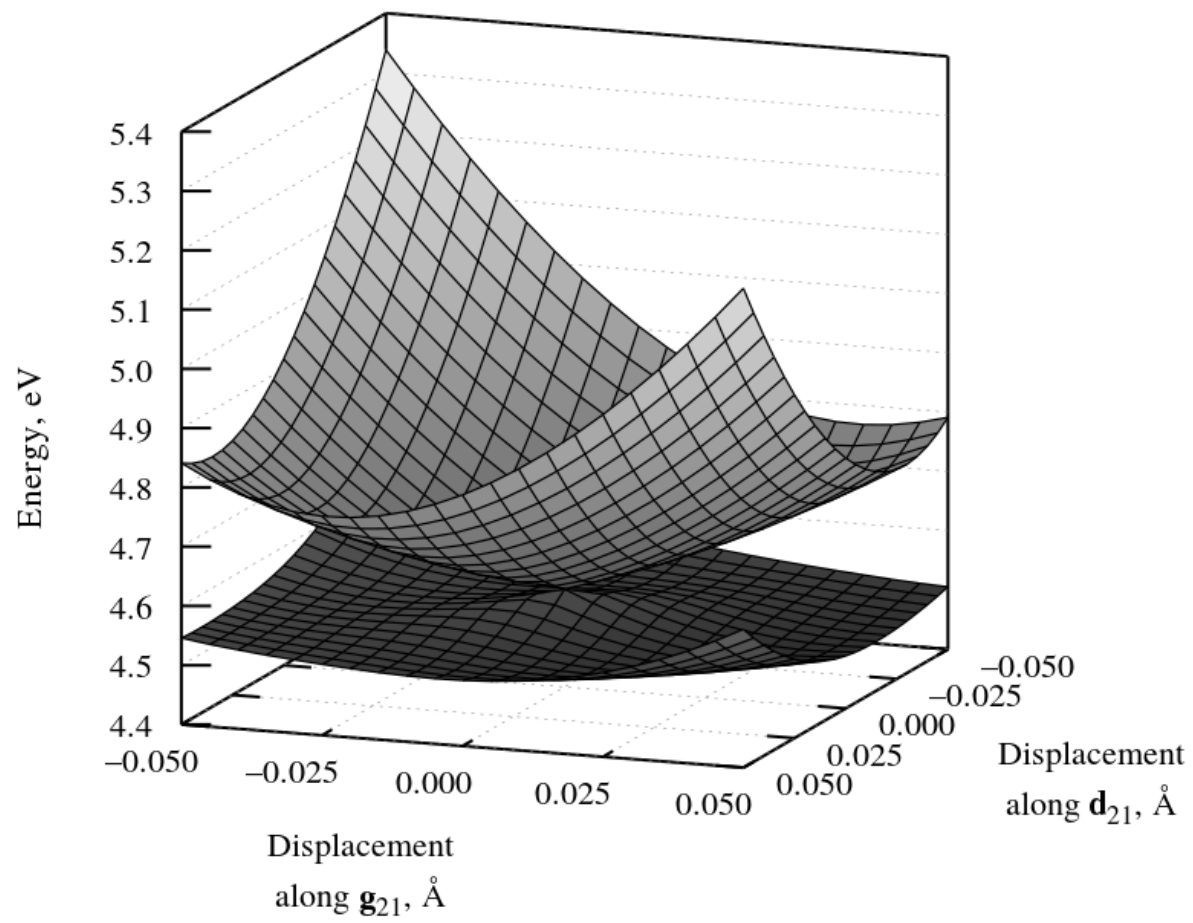

$0.378 \mathrm{eV}$. Evidently, the MCI geometry obtained with SOS-ADC $(2)$ does not coincide with a CI at the XMS-CASPT2 level of theory. This is not surprising, considering how sensitive the excited-state PESs of DMABN are to the level of theory.

The energies of $S_{2}$ and $S_{1}$ states in the branching space of the $S_{2} / S_{1}$ MCI are plotted in Figure S9. It can be seen that the $\operatorname{SOS-ADC}(2)$ method yields a correct topography of the $\mathrm{S}_{2} / \mathrm{S}_{1}$ CI seam. Namely, the PESs of the intersecting states in the immediate vicinity of the MCI exhibit the characteristic double-cone shape. 


\section{S4 Transition State for Isomerization from $\mathrm{S}_{1}$-LE to $\mathrm{S}_{1}$-TICT}

The fact that there are two minima on the $\mathrm{S}_{1}$ PES of DMABN (which is to say, the $\mathrm{S}_{1}$-LE and $\mathrm{S}_{1}$-TICT structures) implies the existence of a transition state along the minimum energy path (MEP) connecting these minima. In order to confirm that the SOS-ADC $(2)$ method can account for there being a transition state on the $\mathrm{S}_{1}$ state, we have optimized its geometry.

The rough location of the transition state was determined with the use of the climbing image variant ${ }^{60}$ of the nudged elastic band algorithm. ${ }^{61,62}$ Subsequently, its geometry was optimized with the 'jobex' script of Turbomole.

The resulting transition state structure, denoted $\mathrm{S}_{1}-\mathrm{TS}$, is shown in Figure S10 (a). As expected, it has a single normal mode with an imaginary frequency, which is $407.42 \mathrm{i} \mathrm{cm}^{-1}$. The atomic displacements corresponding to this mode are shown in Figure S10 (b). It can be seen that this mode corresponds to the pyramidalization of carbon atom $\mathrm{C} 4$, and the rotation of the dimethylamino group around the axis formed by the $\mathrm{C} 4-\mathrm{N} 1$ bond.

Taking into account ZPVE corrections, $\mathrm{S}_{1}$-TS is located $0.202 \mathrm{eV}$ higher in energy than the $\mathrm{S}_{1}-\mathrm{LE}$ minimum. The substantial height of the barrier for isomerization from the $\mathrm{S}_{1}$-LE structure into the $\mathrm{S}_{1}$-TICT structure explains why, under gas-phase conditions, the $\mathrm{S}_{1}$-LE structure is able to trap most of the excited-state population.

Figure S10: (a) Geometry of the $\mathrm{S}_{1}$-TS structure of DMABN. Selected bond lengths are given in units of ångström. (b) Atomic displacements corresponding to the mode with an imaginary frequency.

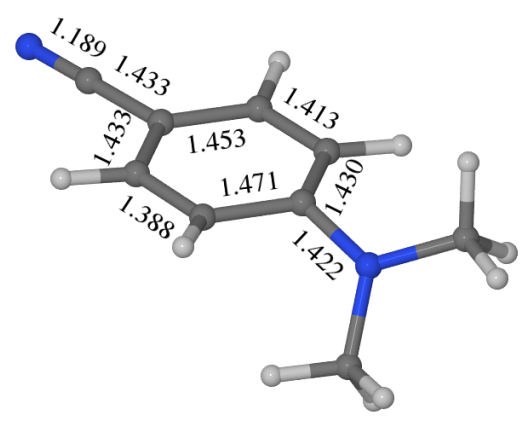

(a) molecular geometry

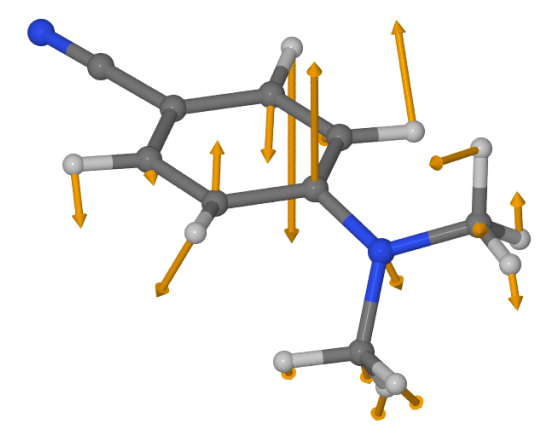

(b) normal mode with imaginary frequency 


\section{S5 Additional Plots of Time-Resolved Fluorescence Spectra}

In the present section, we use a different way of plotting the simulated time-resolved fluorescence spectra of DMABN. For either set of simulations, the spectrum was averaged over 200 fs-long intervals of time (from 0 to $200 \mathrm{fs}$, from 200 to $400 \mathrm{fs}$, and so on). The resulting spectra are shown in Figure S11 on the following page. The assignment of bands is indicated with arrows.

The gas-phase spectrum (Figure S11 (a)) shows two signals. One, which peaks in the range of around 4.3 to $4.8 \mathrm{eV}$, originates from the $\mathrm{S}_{2}$ state. The other, which is mainly localized in the range of around 3.5 to $4.3 \mathrm{eV}$, is the normal fluorescence band of DMABN under gas-phase conditions.

In the water droplet (Figure S11 (b)), an additional fluorescence band appears in the energy range from around 1.5 to around $3.0 \mathrm{eV}$, which is absent the gas phase spectrum. This is the anomalous emission band of DMABN.

Lastly, inspection of Figure S11 (b) shows that the simulation time of 1.2 ps was too short for the fluorescence spectrum of DMABN in the water droplet to fully equilibrate: at $t=1.2 \mathrm{ps}$, the normal band is still decaying in intensity, while the anomalous band is still developing. 
Figure S11: Simulated time-resolved fluorescence spectra of DMABN, averaged over 200 fs-long time intervals, (a) in the gas phase and (b) in the 500-molecule water droplet.

(a) gas phase

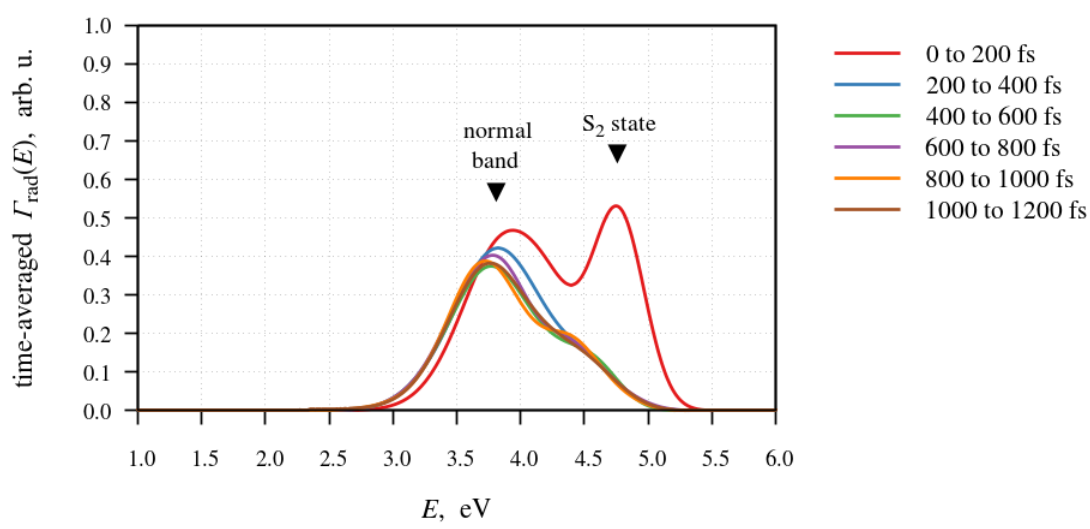

(b) water droplet

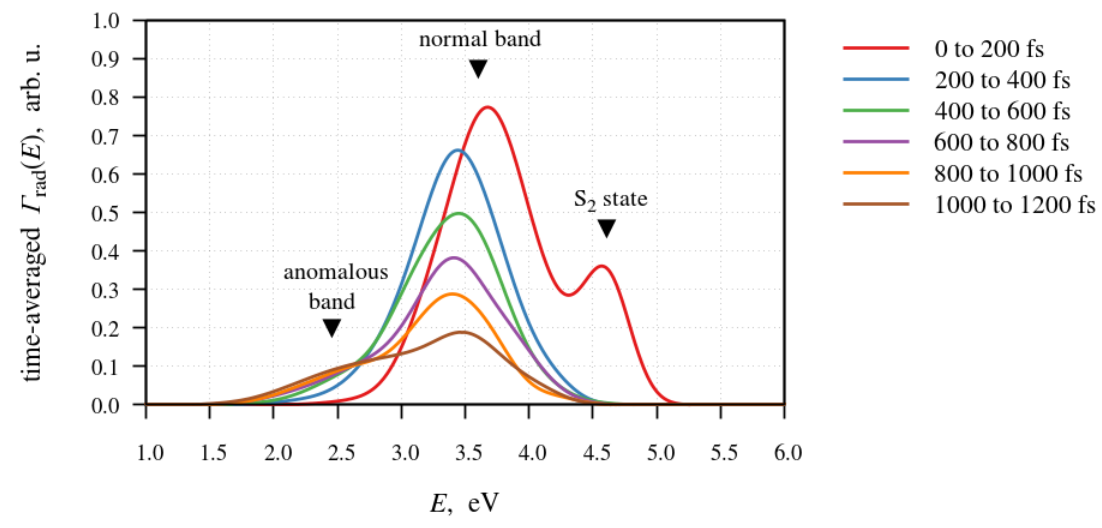




\section{S6 Molecular Geometries}

The present section lists the ground- and excited-state equilibrium geometries of DMABN, as optimized at the SOS-MP2/cc-pVDZ and SOS-ADC(2)/cc-pVDZ levels of theory. All geometries are given in terms of Cartesian coordinates in units of ångström $(\AA)$.

$\mathrm{S}_{0}-\mathrm{GS}$

$\begin{array}{lrrr}\text { C } & 1.2036437 & -1.2137841 & -0.0255932 \\ \text { C } & -0.1958383 & -1.2154715 & -0.0702569 \\ \text { C } & -0.9365380 & -0.0000031 & -0.1083477 \\ \text { C } & -0.1958398 & 1.2154673 & -0.0702865 \\ \text { C } & 1.2036419 & 1.2137842 & -0.0256238 \\ \text { C } & 1.9227665 & 0.0000010 & -0.0039954 \\ \text { C } & 3.3716681 & 0.0000031 & 0.0466211 \\ \text { N } & 4.5531362 & 0.0000055 & 0.0877914 \\ \text { N } & -2.3293568 & -0.0000019 & -0.2026012 \\ \text { C } & -3.0373902 & 1.2395524 & 0.1153633 \\ \mathrm{C} & -3.0373969 & -1.2395536 & 0.1153571 \\ \text { H } & 1.7443320 & -2.1666634 & 0.0000356 \\ \text { H } & -0.7080223 & -2.1804813 & -0.0788113 \\ \text { H } & -0.7080272 & 2.1804749 & -0.0788252 \\ \text { H } & 1.7443270 & 2.1666649 & 0.0000264 \\ \text { H } & -2.7562003 & 2.0395928 & -0.5901265 \\ \text { H } & -2.8415967 & 1.5968572 & 1.1488998 \\ \text { H } & -4.1186447 & 1.0657443 & 0.0015525 \\ \text { H } & -2.7561909 & -2.0395970 & -0.5901220 \\ \text { H } & -4.1186489 & -1.0657462 & 0.0015203 \\ \text { H } & -2.8416263 & -1.5968523 & 1.1489001\end{array}$




$\begin{array}{lrrr}\text { C } & 1.2290690 & -1.2420908 & 0.0459288 \\ \text { C } & -0.2173493 & -1.2302975 & 0.0855512 \\ \text { C } & -0.9043711 & 0.0328786 & 0.0935067 \\ \text { C } & -0.1932606 & 1.2820822 & 0.0557093 \\ \text { C } & 1.2526073 & 1.2650545 & 0.0004947 \\ \text { C } & 1.9289226 & 0.0046111 & 0.0007256 \\ \text { C } & 3.3742615 & -0.0098294 & -0.0465382 \\ \text { N } & 4.5570577 & -0.0216267 & -0.0852147 \\ \text { N } & -2.2854923 & 0.0466464 & 0.1388129 \\ \text { C } & -3.0667663 & 1.2375662 & -0.1995643 \\ \text { C } & -3.0664309 & -1.1288023 & 0.5282101 \\ \text { H } & 1.7842116 & -2.1840982 & 0.0094659 \\ \text { H } & -0.7642167 & -2.1610741 & -0.0917339 \\ \text { H } & -0.7086987 & 2.2234574 & 0.2677860 \\ \text { H } & 1.8276011 & 2.1957907 & 0.0000015 \\ \text { H } & -2.4918499 & 1.8751027 & -0.8852522 \\ \text { H } & -3.3357347 & 1.8107779 & 0.7085551 \\ \text { H } & -3.9952835 & 0.9157135 & -0.7021215 \\ \text { H } & -3.4051807 & -1.6961553 & -0.3600243 \\ \text { H } & -3.9537344 & -0.7889610 & 1.0898937 \\ \text { H } & -2.4607876 & -1.7779160 & 1.1755169\end{array}$




$\begin{array}{lrrr}\text { C } & 1.2128015 & -0.8652149 & 0.5539478 \\ \text { C } & -0.1211783 & -0.4828810 & 0.4577558 \\ \text { C } & -0.4851860 & 0.8098038 & -0.1452320 \\ \text { C } & 0.5955509 & 1.5918031 & -0.7463468 \\ \text { C } & 1.9115439 & 1.1980236 & -0.6073780 \\ \text { C } & 2.2664675 & -0.0474869 & 0.0329445 \\ \text { C } & 3.6377544 & -0.4475084 & 0.1511735 \\ \text { N } & 4.7755307 & -0.7789431 & 0.2458822 \\ \text { N } & -1.6881636 & 0.6626659 & -0.9380093 \\ \text { C } & -1.6419042 & 0.4042236 & -2.3841615 \\ \text { C } & -2.9766493 & 0.4786807 & -0.2737485 \\ \text { H } & 1.4665400 & -1.7920447 & 1.0854945 \\ \text { H } & -0.9055864 & -1.1268404 & 0.8775753 \\ \text { H } & 0.3587800 & 2.5554414 & -1.2194890 \\ \text { H } & 2.7145057 & 1.8436510 & -0.9856098 \\ \text { H } & -0.6742327 & 0.7587868 & -2.7676136 \\ \text { H } & -2.4875425 & 0.9147961 & -2.8753579 \\ \text { H } & -1.7268836 & -0.6875748 & -2.5503783 \\ \text { H } & -3.7455523 & 1.0551416 & -0.8192689 \\ \text { H } & -2.8810973 & 0.8295367 & 0.7626592 \\ \text { H } & -3.2595576 & -0.5906616 & -0.2963257\end{array}$




$\begin{array}{lrrr}\text { C } & 0.2101682 & -0.0401311 & 0.0000000 \\ \mathrm{C} & -0.4838482 & -1.3049352 & 0.0000000 \\ \mathrm{C} & -1.8680413 & -1.3476218 & 0.0000000 \\ \mathrm{C} & -2.6498256 & -0.1516402 & 0.0000000 \\ \mathrm{C} & -1.9555816 & 1.1062720 & 0.0000000 \\ \mathrm{C} & -0.5739713 & 1.1696987 & 0.0000000 \\ \mathrm{~N} & 1.5688656 & 0.0073376 & 0.0000000 \\ \mathrm{C} & -4.1198377 & -0.1851205 & 0.0000000 \\ \mathrm{~N} & -4.8743898 & 0.8212723 & 0.0000000 \\ \mathrm{H} & 0.0807270 & -2.2411281 & 0.0000000 \\ \mathrm{H} & -2.4085967 & -2.3024276 & 0.0000000 \\ \mathrm{H} & -2.5879471 & 2.0038542 & 0.0000000 \\ \mathrm{H} & -0.0793257 & 2.1458408 & 0.0000000 \\ \mathrm{C} & 2.3618205 & -1.2298170 & 0.0000000 \\ \mathrm{C} & 2.2707024 & 1.2987158 & 0.0000000 \\ \mathrm{H} & 2.0143472 & 1.8870039 & 0.8986328 \\ \mathrm{H} & 2.0143472 & 1.8870039 & -0.8986328 \\ \mathrm{H} & 3.3533765 & 1.1109634 & 0.0000000 \\ \mathrm{H} & 2.1494496 & -1.8353120 & 0.8988410 \\ \mathrm{H} & 3.4281114 & -0.9645168 & 0.0000000 \\ \mathrm{H} & 2.1494496 & -1.8353120 & -0.8988410\end{array}$


$\mathrm{S}_{2} / \mathrm{S}_{1} \mathrm{MCI}$

$\begin{array}{lrrr}\text { C } & 1.1683644 & -1.1978349 & -0.1097328 \\ \text { C } & -0.2160338 & -1.2238891 & 0.1420752 \\ \text { C } & -0.9036867 & 0.0232266 & 0.4428840 \\ \text { C } & -0.1642472 & 1.2540835 & 0.5404879 \\ \text { C } & 1.2314804 & 1.2642647 & 0.3341878 \\ \text { C } & 1.9044029 & 0.0377998 & -0.0294495 \\ \text { C } & 3.3094783 & 0.0574210 & -0.3123846 \\ \text { N } & 4.4728593 & 0.0871097 & -0.5504694 \\ \text { N } & -2.3089822 & 0.0843001 & 0.3429819 \\ \text { C } & -2.9441096 & 1.0354653 & -0.5741256 \\ \mathrm{C} & -3.1288998 & -1.0428244 & 0.7748474 \\ \text { H } & 1.6968651 & -2.1205605 & -0.3753730 \\ \text { H } & -0.7741005 & -2.1612275 & 0.0425625 \\ \text { H } & -0.6810454 & 2.1648064 & 0.8672249 \\ \text { H } & 1.8035208 & 2.1914645 & 0.4402018 \\ \text { H } & -2.3006988 & 1.9205654 & -0.6836376 \\ \text { H } & -3.9402355 & 1.3217946 & -0.1944698 \\ \text { H } & -3.0540094 & 0.5591491 & -1.5714970 \\ \text { H } & -3.2383526 & -1.7863150 & -0.0398690 \\ \text { H } & -4.1339948 & -0.6742370 & 1.0462408 \\ \text { H } & -2.6540399 & -1.5202596 & 1.6446397\end{array}$




$\begin{array}{lrrr}\text { C } & 1.1658683 & -1.2051737 & -0.1423456 \\ \text { C } & -0.2246178 & -1.1939070 & 0.1084852 \\ \text { C } & -0.9011206 & 0.0054684 & 0.4935693 \\ \text { C } & -0.1617718 & 1.2803389 & 0.5398166 \\ \text { C } & 1.2054498 & 1.2510902 & 0.3006674 \\ \text { C } & 1.8936617 & 0.0506566 & -0.0720237 \\ \text { C } & 3.3028936 & 0.0907496 & -0.3296265 \\ \text { N } & 4.4728520 & 0.1147782 & -0.5403584 \\ \text { N } & -2.3129209 & 0.0575019 & 0.3332748 \\ \text { C } & -2.9229482 & 1.0237281 & -0.5835031 \\ \text { C } & -3.1473645 & -1.0508177 & 0.7815629 \\ \text { H } & 1.6988702 & -2.1502542 & -0.2936515 \\ \text { H } & -0.7942455 & -2.1268188 & 0.0017099 \\ \text { H } & -0.6373402 & 2.1740475 & 0.9632390 \\ \text { H } & 1.7924520 & 2.1720211 & 0.4099094 \\ \text { H } & -2.2537865 & 1.8930815 & -0.6752415 \\ \text { H } & -3.9164423 & 1.3193418 & -0.2068204 \\ \text { H } & -3.0334973 & 0.5571632 & -1.5848189 \\ \text { H } & -3.2565956 & -1.8060143 & -0.0232668 \\ \text { H } & -4.1501707 & -0.6671554 & 1.0372264 \\ \text { H } & -2.6746911 & -1.5155230 & 1.6595210\end{array}$




\section{References}

[1] Friesner, R. A.; Guallar, V. Ab Initio Quantum Chemical and Mixed Quantum Mechanics/Molecular Mechanics (QM/MM) Methods for Studying Enzymatic Catalysis. Annu. Rev. Phys. Chem. 2005, 56, 389-427. DOI: 10.1146/annurev.physchem.55.091602.094410

[2] Lin, H.; Truhlar, D. G. QM/MM: What Have We Learned, Where Are We, and Where Do We Go From Here? Theor. Chem. Acc. 2007, 117, 185-199. DOI: $10.1007 / \mathrm{s} 00214-006-0143-\mathrm{Z}$

[3] Senn, H. M.; Thiel, W. QM/MM Methods for Biological Systems. Top. Curr. Chem. 2007, 268, 173-290. DOI: 10.1007/128_2006_084

[4] Schmitt, U. W.; Voth, G. A. The Computer Simulation of Proton Transport in Water. J. Chem. Phys. 1999, 111, 9361-9381. DOI: 10.1063/1.480032

[5] Jorgensen, W. L.; Maxwell, D. S.; Tirado-Rives, J. Development and Testing of the OPLS All-Atom Force Field on Conformational Energetics and Properties of Organic Liquids. J. Am. Chem. Soc. 1996, 118, 11225-11236. DOI: $10.1021 /$ ja9621760

[6] Ruckenbauer, M.; Barbatti, M.; Müller, T.; Lischka, H. Nonadiabatic Excited-State Dynamics with Hybrid ab Initio Quantum-Mechanical/MolecularMechanical Methods: Solvation of the Pentadieniminium Cation in Apolar Media. J. Phys. Chem. A 2010, 114, 6757-6765. DOI: 10.1021/jp103101t

[7] Ruckenbauer, M.; Barbatti, M.; Müller, T.; Lischka, H. Nonadiabatic Photodynamics of a Retinal Model in Polar and Nonpolar Environment. J. Phys. Chem. A 2013, 117, 2790-2799. DOI: 0.1021/jp400401f

[8] Barbatti, M.; Kakali, S. Effects of Different Initial Condition Samplings on Photodynamics and Spectrum of Pyrrole. Int. J. Quantum Chem. 2016, 116, 762-771. DOI: 10.1002/qua.25049

[9] Mai, S.; Gattuso, H.; Monari, A.; González, L. Novel Molecular-Dynamics-Based Protocols for Phase Space Sampling in Complex Systems. Front. Chem. 2018, 6, 495. DOI: $10.3389 /$ fchem.2018.00495

[10] Bowman, J. M.; Gazdy, B.; Sun, Q. A Method to Constrain Vibrational Energy in Quasiclassical Trajectory Calculations. J. Chem. Phys. 1989, 91, 2859-2862. DOI: $10.1063 / 1.456955$

[11] Guo, Y.; Thompson, D. L.; Sewell, T. D. Analysis of the Zero-Point Energy Problem in Classical Trajectory Simulations. J. Chem. Phys. 1996, 104, 576-582. DOI: $10.1063 / 1.470853$ 
[12] Martínez-Fernández, L.; Corral, I.; Granucci, G.; Persico, M. Competing Ultrafast Intersystem Crossing and Internal Conversion: A Time Resolved Picture for the Deactivation of 6-Thioguanine. Chem. Sci. 2014, 5, 1336-1347. DOI: $10.1039 / \mathrm{c} 3 \mathrm{sc} 52856 \mathrm{a}$

[13] Singh, U. C.; Kollman, P. A. An Approach to Computing Electrostatic Charges for Molecules. J. Comp. Chem. 1984, 5, 129-145. DOI: 10.1002/jcc.540050204

[14] Gaussian 16, Revision B.01, Frisch, M. J.; Trucks, G. W.; Schlegel, H. B.; Scuseria, G. E.; Robb, M. A.; Cheeseman, J. R.; Scalmani, G.; Barone, V.; Mennucci, B.; Petersson, G. A. et al., Gaussian, Inc., Wallingford CT, 2016.

[15] Brünger, A.; Brooks III, C. L.; Karplus, M. Stochastic Boundary Conditions for Molecular Dynamics Simulations of ST2 Water. Chem. Phys. Lett. 1984, 105, 495-500. DOI: 10.1016/0009-2614(84)80098-6

[16] Barbatti, M.; Aquino, A. J. A.; Lischka, H. The UV absorption of Nucleobases: Semi-Classical Ab Initio Spectra Simulations. Phys. Chem. Chem. Phys. 2010, 12, 4959-4967. DOI: 10.1039/B924956G

[17] Crespo-Otero, R.; Barbatti, M. Spectrum Simulation and Decomposition with Nuclear Ensemble: Formal Derivation and Application to Benzene, Furan and 2-Phenylfuran. Theor. Chem. Acc. 2012, 131, 1237. DOI: 10.1007/s00214-012$1237-4$

[18] Barbatti, M.; Granucci, G.; Ruckenbauer, M.; Plasser, F.; Crespo-Otero, R.; Pittner, J.; Persico, M.; Lischka, H. NEWTON-X: a package for Newtonian dynamics close to the crossing seam, version 2.0, www.newtonx.org (2017).

[19] Plasser, F.; Crespo-Otero, R.; Pederzoli, M.; Pittner, J.; Lischka, H.; Barbatti, M. Surface Hopping Dynamics with Correlated Single-Reference Methods: $9 \mathrm{H}-$ Adenine as a Case Study. J. Chem. Theory Comput. 2014, 10, 1395-1405. DOI: $10.1021 / \operatorname{ct} 4011079$

[20] Barbatti, M.; Granucci, G.; Persico, M.; Ruckenbauer, M.; Vazdar, M.; Eckert-Maksic, M.; Lischka, H. The On-the-Fly Surface-Hopping Program System Newton-X: Application to Ab Initio Simulation of the Nonadiabatic Photodynamics of Benchmark Systems. J. Photochem. Photobiol., A 2007, 190, 228-240. DOI: 10.1016/j.jphotochem.2006.12.008

[21] Barbatti, M.; Ruckenbauer, M.; Plasser, F.; Pittner, J.; Granucci, G.; Persico, M.; Lischka, H. Newton-X: a Surface-Hopping Program for Nonadiabatic Molecular Dynamics. Wiley Interdiscip. Rev.: Comput. Mol. Sci. 2014, 4, 26-33. DOI: $10.1002 /$ wcms.1158

[22] Tavernelli, I.; Tapavicza, E.; Rothlisberger, U. Nonadiabatic Coupling Vectors Within Linear Response Time-Dependent Density Functional Theory. J. Chem. Phys. 2009, 130, 124107. DOI: 10.1063/1.3097192 
[23] Plasser, F.; Ruckenbauer, M.; Mai, S.; Oppel, M.; Marquetand, P.; González, L. Efficient and Flexible Computation of Many-Electron Wavefunction Overlaps. J. Chem. Theory Comput. 2016, 12, 1207-1219. DOI: 10.1021/acs.jctc.5b01148

[24] Plasser, F.; González, L. Communication: Unambiguous Comparison of ManyElectron Wavefunctions Through Their Overlaps. J. Chem. Phys. 2003, 145, 021103. DOI: $10.1063 / 1.4958462$

[25] Granucci, G.; Persico, M. Critical Appraisal of the Fewest Switches Algorithm for Surface Hopping. J. Chem. Phys. 2007, 126, 134114. DOI: 10.1063/1.2715585

[26] Kochman, M. A.; Tajti, A.; Morrison, C. A.; Miller, R. J. D. Early Events in the Nonadiabatic Relaxation Dynamics of $4-(N, N$-Dimethylamino $)$ benzonitrile. J. Chem. Theory Comput. 2015, 11, 1118-1128. DOI: 10.1021/ct5010609

[27] Wiggins, P.; Williams, J. A. G.; Tozer, D. J. Excited State Surfaces in Density Functional Theory: A New Twist on an Old Problem. J. Chem. Phys. 2009, 131, 091101. DOI: $10.1063 / 1.3222641$

[28] Hättig, C. Structure Optimizations for Excited States with Correlated SecondOrder Methods: $\mathrm{CC} 2$ and $\mathrm{ADC}(2)$. In Advances in Quantum Chemistry; Jensen, H. J. Å.., Ed.; Academic Press: New York, 2005; Vol. 50; pp 37-60. DOI: $10.1016 /$ S0065-3276(05)50003-0.

[29] Köhn, A.; Hättig, C. On the Nature of the Low-Lying Singlet States of 4-(Dimethyl-amino)benzonitrile. J. Am. Chem. Soc. 2004, 126, 7399-7410. DOI: $10.1021 / \mathrm{ja} 0490572$

[30] Gómez, I.; Castro, P. J.; Reguero, M. Insight into the Mechanisms of Luminescence of Aminobenzonitrile and Dimethylaminobenzonitrile in Polar Solvents. An ab Initio Study. J. Phys. Chem. A 2015, 119, 1983-1995. DOI: $10.1021 /$ acs.jpca.5b01421

[31] Mewes, S. A.; Plasser, F.; Krylov, A.; Dreuw, A. Benchmarking Excited-State Calculations Using Exciton Properties. J. Chem. Theory Comput. 2018, 14, 710725. DOI: $10.1021 /$ acs.jctc.7b01145

[32] Grimme, S. Improved Second-Order Møller-Plesset Perturbation Theory by Separate Scaling of Parallel- and Antiparallel-Spin Pair Correlation. Energies J. Chem. Phys. 2003, 118, 9095-9102. DOI: 10.1063/1.1569242

[33] Grimme, S.; Goerigk, L.; Fink, R. F. Spin-Component-Scaled Electron Correlation Methods. WIREs Comput. Mol. Sci. 2012, 2, 886-906. DOI: $10.1002 /$ wcms.1110

[34] Jung, Y.; Lochan, R. C.; Dutoi, A. D.; Head-Gordon, M. Scaled OppositeSpin Second Order Møller-Plesset Correlation Energy: An Economical 
Electronic Structure Method. J. Chem. Phys. 2004, 121, 9793-9802. DOI: $10.1063 / 1.1809602$

[35] Grimme, S.; Izgorodina, E. I. Calculation of 0-0 Excitation Energies of Organic Molecules by CIS(D) Quantum Chemical Methods. Chem. Phys. 2004, 305, 223-230. DOI: 10.1016/j.chemphys.2004.06.050

[36] Rhee, Y. M.; Head-Gordon, M. Scaled Second-Order Perturbation Corrections to Configuration Interaction Singles: Efficient and Reliable Excitation Energy Methods. J. Phys. Chem. A 2007, 111, 5314-5326. DOI: 10.1021/jp068409j

[37] Hellweg, A.; Grün, S. A.; Hättig, C. Benchmarking the Performance of Spin-Component Scaled CC2 in Ground and Electronically Excited States. Phys. Chem. Chem. Phys. 2008, 10, 4119-4127. DOI: 10.1039/b803727b

[38] Casanova, D.; Rhee, Y. M.; Head-Gordon, M. Quasidegenerate Scaled Opposite Spin Second Order Perturbation Corrections to Single Excitation Configuration Interaction. J. Chem. Phys. 2008, 128, 164106. DOI: 10.1063/1.2907724

[39] Winter, N. O. C.; Hättig, C. Scaled Opposite-Spin CC2 for Ground and Excited States with Fourth Order Scaling Computational Costs. J. Chem. Phys. 2011, 134, 184101. DOI: 10.1063/1.3584177

[40] Krauter, C. M.; Pernpointner, M.; Dreuw, A. Application of the Scaled-Opposite-Spin Approximation to Algebraic Diagrammatic Construction Schemes of Second Order. J. Chem. Phys. 2013, 138, 044107. DOI: $10.1063 / 1.4776675$

[41] Christiansen, O.; Koch, H.; Jørgensen, P. Perturbative Triple Excitation Corrections to Coupled Cluster Singles and Doubles Excitation Energies. J. Chem. Phys. 1996, 105, 1451-1459. DOI: 10.1063/1.472007

[42] Kochman, M. A.; Bil, A.; Dwayne Miller, R. J. Mechanism Underlying the Nucleobase-Distinguishing Ability of Benzopyridopyrimidine (BPP). J. Phys. Chem. A 2017, 121, 8267-8279. DOI: 10.1021/acs.jpca.7b08334

[43] Kendall, R. A.; Dunning, Jr., T. H.; Harrison, R. J. Electron Affinities of the First-Row Atoms Revisited. Systematic Basis Sets and Wave Functions. J. Chem. Phys. 1992, 96, 6796-6806. DOI: 10.1063/1.462569

[44] Harbach, P. H. P.; Wormit, M.; Dreuw, A. The Third-Order Algebraic Diagrammatic Construction Method $(\mathrm{ADC}(3))$ for the Polarization Propagator for Closed-Shell Molecules: Efficient Implementation and Benchmarking. $J$. Chem. Phys. 2014, 141, 064113. DOI: 10.1063/1.4892418

[45] Dreuw, A.; Wormit, M. The Algebraic Diagrammatic Construction Scheme for the Polarization Propagator for the Calculation of Excited States. WIREs Comput. Mol. Sci. 2015, 5, 82-95. DOI: 10.1002/wcms.1206 
[46] https://adc-connect.org/v0.13.3/index.html, Accessed on January 8, 2020.

[47] Herbst, M. F.; Scheurer, M.; Fransson, T.; Rehn, D. R.; Dreuw, A. adcc: A versatile toolkit for rapid development of algebraic-diagrammatic construction methods. https://hal.archives-ouvertes.fr/hal-02319517

[48] Herbst, M. F.; Scheurer, M. adc-connect/adcc v0.13.4 (Version v0.13.4). 2020 Zenodo. http://doi.org/10.5281/zenodo.3631806

[49] Krishnan, R.; Binkley, J. S.; Seeger, R.; Pople, J. A. Self-Consistent Molecular Orbital Methods. XX. A Basis Set for Correlated Wave Functions J. Chem. Phys. 1980, 72, 650-654. DOI: 10.1063/1.438955

[50] Sun, Q.; Berkelbach, T. C.; Blunt, N. S.; Booth, G. H.; Guo, S.; Li, Z.; Liu, J.; McClain, J. D.; Sayfutyarova, E. R.; Sharma, S.; Wouters, S.; Chan, G. K.-L. PySCF: the Python-Based Simulations of Chemistry Framework. WIREs Comput. Mol. Sci. 2018, 8, e1340. DOI: 10.1002/wcms.1340

[51] Martin, R. L. Natural Transition Orbitals. J. Chem. Phys. 2003, 118, 4775-4777. DOI: $10.1063 / 1.1558471$

[52] Ciminelli, C.; Granucci, G.; Persico, M. The Photoisomerization Mechanism of Azobenzene: A Semiclassical Simulation of Non-adiabatic Dynamics. Chem. Eur. J. 2004, 10, 2327-2341, DOI: 10.1002/chem.200305415

[53] Shiozaki, T.; Győrffy, W.; Celani, P.; Werner, H.-J. Communication: Extended Multi-State Complete Active Space Second-Order Perturbation Theory: Energy and Nuclear Gradients. J. Chem. Phys. 2011, 135, 081106. DOI: $10.1063 / 1.3633329$

[54] BAGEL, Brilliantly Advanced General Electronic-structure Library. http://www.nubakery.org under the GNU General Public License.

[55] Shiozaki, T. BAGEL: Brilliantly Advanced General Electronic-structure Library. WIREs Comput. Mol. Sci. 2017, e1331. DOI: 10.1002/wcms.1331

[56] Roos, B. O. The Complete Active Space Self-Consistent Field Method and its Applications in Electronic Structure Calculations. Adv. Chem. Phys. 1987, 69, 399-445. DOI: 10.1002/9780470142943.ch7

[57] Finley, J.; Malmqvist, P.-A.; Roos, B. O.; Serrano-Andrés, L. The Multi-State CASPT2 Method. Chem. Phys. Lett. 1998, 288, 299-306. DOI: 10.1016/S00092614(98)00252-8

[58] Gómez, I.; Reguero, M.; Boggio-Pasqua, M.; Robb, M. A. Intramolecular Charge Transfer in 4-Aminobenzonitriles Does Not Necessarily Need the Twist. J. Am. Chem. Soc. 2005, 127, 7119-7129. DOI: 10.1021/ja042413w 
[59] Georgieva, I.; Aquino, A. J. A.; Plasser, F.; Trendafilova, N.; Köhn, A.; Lischka, H. Intramolecular Charge-Transfer Excited-State Processes in 4( $N, N$-Dimethylamino)benzonitrile: The Role of Twisting and the $\pi \sigma^{*}$ State. J. Phys. Chem. A 2015, 119, 6232-6243. DOI: 10.1021/acs.jpca.5b03282

[60] Henkelman, G.; Uberuaga, B. P.; Jósson, H. A Climbing Image Nudged Elastic Band Method for Finding Saddle Points and Minimum Energy Paths. J. Chem. Phys. 2000, 113, 9901-9904. DOI: 10.1063/1.1329672

[61] Jósson, H.; Mills, G.; Jacobsen, K. W. Nudged Elastic Band Method for Finding Minimum Energy Paths of Transitions. In Classical and Quantum Dynamics in Condensed Phase Simulations; Berne, B. J.; Ciccotti, G.; Coker, D. F., Eds.; World Scientific, 2004; pp 385-404.

[62] Henkelman, G.; Jósson, H. Improved Tangent Estimate in the Nudged Elastic Band Method for Finding Minimum Energy Paths and Saddle Points. J. Chem. Phys. 2000, 113, 9978-9985. DOI: 10.1063/1.1323224 